\title{
Light top partners and precision physics
}

\author{
Christophe Grojean, ${ }^{a, c}$ Oleksii Matsedonskyi ${ }^{b}$ and Giuliano Panico ${ }^{c}$ \\ ${ }^{a}$ ICREA at IFAE, Universitat Autònoma de Barcelona, \\ 08193 Bellaterra, Barcelona, Spain \\ ${ }^{b}$ Dipartimento di Fisica e Astronomia and INFN, Sezione di Padova, \\ Via Marzolo 8, I-35131 Padova, Italy \\ ${ }^{c}$ Theory Division, Physics Department, CERN, \\ CH-1211, Geneva 23, Switzerland \\ E-mail: Christophe.Grojean@cern.ch, matsedonskyi@pd.infn.it, \\ giuliano.panico@cern.ch
}

ABSTRACT: We analyze the corrections to the precision EW observables in minimal composite Higgs models by using a general effective parametrization that also includes the lightest fermionic resonances. A new, possibly large, logarithmically divergent contribution to $\widehat{S}$ is identified, which comes purely from the strong dynamics. It can be interpreted as a running of $\widehat{S}$ induced by the non-renormalizable Higgs interactions due to the nonlinear $\sigma$-model structure. As expected, the corrections to the $\widehat{T}$ parameter coming from fermion loops are finite and dominated by the contributions of the lightest composite states. The fit of the oblique parameters suggests a rather stringent lower bound on the $\sigma$-model scale $f \gtrsim 750 \mathrm{GeV}$. The corrections to the $Z \bar{b}_{L} b_{L}$ vertex coming from the lowest-order operators in the effective Lagrangian are finite and somewhat correlated to the corrections to $\widehat{T}$. Large additional contributions are generated by contact interactions with 4 composite fermions. In this case a logarithmic divergence can be generated and the correlation with $\widehat{T}$ is removed. We also analyze the tree-level corrections to the top couplings, which are expected to be large due to the sizable degree of compositeness of the third generation quarks. We find that for a moderate amount of tuning the deviation in $V_{t b}$ can be of order $5 \%$ while the distortion of the $Z \bar{t}_{L} t_{L}$ vertex can be $10 \%$.

Keywords: Higgs Physics, Beyond Standard Model, Technicolor and Composite Models 


\section{Contents}

1 Introduction 1

2 The model 3

2.1 The effective Lagrangian 4

3 General analysis of the EW parameters $\quad 7$

$\begin{array}{lll}3.1 & \text { The oblique parameters } & 7\end{array}$

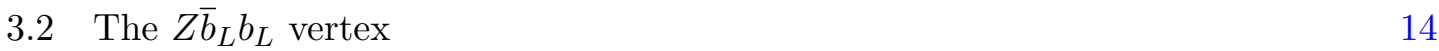

$\begin{array}{lll}3.3 & \text { Symmetries in the effective Lagrangian } & 19\end{array}$

4 Results in explicit models 20

4.1 The case of a light singlet 20

4.2 The case of a light 4-plet 23

4.3 Two complete models 26

5 The case of a totally composite $t_{R} \quad 31$

5.1 The effective Lagrangian 31

5.2 Results 32

6 Corrections to the top couplings $\quad 36$

6.1 A relation between $\delta g_{t_{L}}$ and $\delta V_{t b} \quad 37$

6.2 The case of an elementary $t_{R} \quad 38$

6.3 The case of a composite $t_{R} \quad 39$

$\begin{array}{lll}7 & \text { Conclusions } & 40\end{array}$

A The CCWZ notation $\quad 42$

B Operator analysis for the $Z \bar{b}_{L} b_{L}$ vertex 45

C Computation of the loop corrections to the $Z \bar{b}_{L} b_{L}$ vertex 49

\section{Introduction}

The discovery $[1,2]$ by the LHC experiments of a scalar resonance with a mass around $125 \mathrm{GeV}$ and with production and decay properties compatible with the ones of the Standard Model (SM) Higgs boson sets a landmark in the exploration of the sector responsible for the breaking of the Electroweak (EW) symmetry. The value of the resonance mass together with the absence of observation of any additonal new particles bring stringent 
constraints on various models that were designed to address the naturalness problem. For instance, the current results and in particular the lack of any signal in the jets plus missing energy searches, in addition to the indirect constraints from flavor physics, indicate that supersymmetric models are in need for a non-minimal incarnation (see for instance ref. [3] and references therein). Important lessons can also be drawn for models of strong electroweak symmetry breaking in which the Higgs boson emerges as a composite particle [4-11] (see also ref. [12]). An interesting result is the fact that light fermionic top partners below $1 \mathrm{TeV}$ are necessary to generate the correct Higgs mass without too much fine-tuning [1318]. This forces also the composite Higgs models into non-minimal territory with some fermionic resonances below the expected typical mass scale of the resonances of the strong sector. Moreover it motivates an extension of the effective description proposed in ref. [19] in order to include the appropriate dynamics and couplings of the light top partners.

It has been realized that light fermionic top partners offer nice distinctive collider signatures of composite Higgs models and the best search strategies at the LHC have been identified [20-42] and are being applied by the experimental collaborations [43-46]. Including the light fermionic resonances in a general effective Lagrangian, as we will do in this work, can also provide a model-independent tool to study these collider signatures.

A third and essential motivation to consider an effective description of top partners is to reassess the status of the composite Higgs models regarding the EW constraints. The composite nature of the Higgs is indeed the source of an infrared-saturated contribution to the EW oblique parameters [47] that, taken on its own, sets a stringent bound on the compositeness scale of the Higgs boson and inevitably raises the amount of fine-tuning [4851]. It is thus clear that a scenario with an acceptable amount of tuning can only be obtained if further corrections to the EW parameters are present.

One possible source of additional contributions are the composite resonances and in particular the fermionic ones. Even if they do not give tree-level corrections to the EW oblique parameters, the top partners do contribute to them at one loop and these contributions can be sizable if the partners are light. In this paper we extend previous analyses $[47,52-58]$ and we provide the first computation of the fermion one-loop contribution to the $\widehat{S}$ parameter taking into account the Higgs non-linearities associated to its composite nature. The result of this computation is the identification of a new logarithmically enhanced contribution that can be interpreted as a running effect from the mass of the top partners to the scale of the EW vector resonances. We also study the contributions of the top partners to the $\widehat{T}$ parameter which, though finite, can be large and positive, in particular in the presence of a light $\mathrm{SU}(2)$ singlet partner, and can compensate the Higgs contribution. We also clarify the structure of the deviations of the $Z \bar{b}_{L} b_{L}$ coupling which can become logarithmically divergent when 4 -fermion interactions with a chirality structure LLRR are introduced in the composite sector.

This paper is organized as follows. In section 2 we present the effective Lagrangian describing a composite Higgs as Goldstone boson associated to the coset $\mathrm{SO}(5) / \mathrm{SO}(4)$ together with the light top partners and their couplings to the SM fermions. In section 3 we present a general analysis of the corrections to the EW observables. In particular we estimate the contributions of the top partners to the EW oblique parameters and to the 
deviations of the couplings of the $Z$ gauge boson to the $b$ quark. Section 4 is devoted to the numerical analysis of some explicit models. In section 5 we repeat the previous analysis within an alternative set-up in which the $t_{R}$ appears as a completely composite state. And finally in section 6 we compute the modifications of the couplings of the top quark induced by the mixing with its partners. Afterwards we conclude in section 7 . The appendices resume our conventions and collect a few technical details.

\section{The model}

The first step in our analysis of the EW precision constraints is the identification of a suitable parametrization of the composite Higgs models. As explained in the Introduction, our strategy is to study the new physics effects on the EW parameters from a low-energy perspective. The main advantage of this approach is the possibility to capture the main features of the composite Higgs scenario and to describe a broad class of explicit models in a unified framework.

The fundamental ingredient of the composite Higgs scenario is the identification of the Higgs boson with a set of Goldstones coming from the spontaneous breaking of a global symmetry of a new strongly-coupled dynamics. For definiteness, in the following we will focus on the case in which the Goldstone bosons are associated to the coset $\mathrm{SO}(5) / \mathrm{SO}(4)$. This is the minimal choice that gives rise to only one Higgs doublet and contains an $\mathrm{SO}(3)_{c}$ custodial symmetry. As we will see, the presence of a global symmetry in the composite sector strongly constrains the structure of the effective Lagrangian and in particular fixes the form of the Goldstones interactions.

In this paper we will be mainly interested in the corrections to the EW observables that come from the presence of light fermionic resonances. To analyze this aspect we will construct an effective description of the composite models in which only the light fermionic states coming from the strong sector are included, while the heavier fermionic states and the bosonic resonances are integrated out. We associate to the heavy resonances a typical mass scale $m_{*}$, which can be interpreted as the cut-off of our effective theory. In a generic strongly coupled sector $m_{*}$ is connected to the coupling of the strong dynamics $g_{*}$ and to the Goldstone decay constant $f$ by the relation $m_{*} \simeq g_{*} f$ [19]. Of course our effective description is valid as far as there is a mass gap between the light and the heavy resonances $m_{\text {light }} \ll m_{*}$.

In the usual framework of composite Higgs models the SM fields do not come from the strong dynamics, instead they are introduced as elementary states external with respect to the composite sector. The elementary fermions are mixed to the composite dynamics following the assumption of partial compositeness [59, 60], which requires that they have only linear mixing with the operators coming from the strong sector. For simplicity we only include the top quark in our effective description. It is the field that has the largest mixing with the composite states and induces the most important corrections to the EW observables. The mixing of the elementary doublet $q_{L}$ and of the singlet $t_{R}$ can be schematically written as

$$
\mathcal{L}_{\text {mix }}=y_{L} \bar{q}_{L} \mathcal{O}_{L}+y_{R} \bar{t}_{R} \mathcal{O}_{R}+\text { h.c. }
$$


where $\mathcal{O}_{L, R}$ are operators coming from the strong dynamics. An important point is the fact that global $\mathrm{SO}(5)$ symmetry of the strong sector is unbroken in the UV where the elementary-composite mixings are generated, thus the composite operators $\mathcal{O}_{L, R}$ will belong to some liner representation of $\mathrm{SO}(5)$. On the other hand, the elementary states transform only under the SM gauge group and they do not fill complete $\mathrm{SO}(5)$ representations. The mixing between elementary and composite states induces a (small) explicit breaking of the global $\mathrm{SO}(5)$ invariance, making the Higgs a pseudo Goldstone boson and generating an effective Higgs potential. From a low-energy perspective, the mixing in eq. (2.1) can be reinterpreted as a linear mixing between the elementary states and some fermionic resonances $\Psi$ coming from the strong dynamics:

$$
\mathcal{L}_{\text {mix }}^{\mathrm{eff}}=y_{L} f \bar{q}_{L} \Psi_{R}+y_{R} f \bar{t}_{R} \Psi_{L}+\text { h.c. } .
$$

The assumption of partial compositeness also determines the coupling of the elementary gauge fields with the composite sector. The SM gauge fields are coupled to the strong dynamics via the weak gauging of a subgroup of the global invariance. As well known, in order to accommodate the correct hypercharges of the SM fermions, an extra Abelian subgroup must be added to the global invariance of the composite sector, which becomes $\mathrm{SO}(5) \times \mathrm{U}(1)_{X}$. The $\mathrm{SM} \mathrm{SU}(2)_{L}$ group is identified with the corresponding factor of the $\mathrm{SO}(4) \simeq \mathrm{SU}(2)_{L} \times \mathrm{SU}(2)_{R}$ subgroup of $\mathrm{SO}(5)$, while the hypercharge generator corresponds to the combination $Y=T_{R}^{3}+X$, where $T_{R}^{3}$ is the third generator of $\mathrm{SU}(2)_{R}$ (see appendix A for further details and for our conventions). The weak gauging induces another small explicit breaking of the global $\mathrm{SO}(5)$ symmetry. This breaking is however typically subleading with respect to the one induced by the top quark mixing.

\subsection{The effective Lagrangian}

We can now discuss in more details the structure of the effective theory and derive the general form of the effective Lagrangian respecting our basic assumptions. In our derivation we will follow the standard CCWZ approach [61, 62], which allows to build all the operators in the effective Lagrangian starting from elements in irreducible representations of the unbroken global group $\mathrm{SO}(4)$.

The Higgs doublet is described by the set of 4 Goldstone bosons $\Pi_{i}$ encoded in the Goldstone matrix $U$,

$$
U \equiv \exp \left[i \frac{\sqrt{2}}{f} \Pi_{i} T^{i}\right],
$$

where $T^{i}(i=1, \ldots, 4)$ are the generators of the $\mathrm{SO}(5) / \mathrm{SO}(4)$ coset. The operators in the effective Lagrangian can be written in terms of the $U$ matrix and of the CCWZ operators $e_{\mu}$ and $d_{\mu}$, that come from the covariant derivative of the Goldstone matrix (see appendix A for further details). The $e_{\mu}$ symbol is used to build the covariant derivative of the composite fermions. The $d_{\mu}$ symbol transforms as a 4-plet of $\mathrm{SO}(4)$ and enters in the kinetic terms for the Goldstones, which read

$$
\mathcal{L}_{\text {gold }}=\frac{f^{2}}{4} d_{\mu}^{i} d_{i}^{\mu}
$$


The fermion sector of the theory depends on the quantum numbers we choose for the composite sector operators $\mathcal{O}_{L, R}$. In the following we will concentrate on the case in which the operators belong to the fundamental representation of $\mathrm{SO}(5)$. With this choice we are able to parametrize the low-energy dynamics of several explicit models proposed in the literature (see for example refs. [27, 63-67]). The requirement of a mixing with the elementary top quark fixes the $\mathrm{U}(1)_{X}$ charge of these operators to be $2 / 3$.

As mentioned before, in the effective theory we can describe the low-energy dynamics of the strong sector through a set of fermionic states. For simplicity we include only one level of composite fermions in our effective description and we identify the cut-off with the mass of the lightest of the other resonances. In the CCWZ approach the fields are introduced as irreducible representations of the unbroken group $\mathrm{SO}(4)$ and transform nonlinearly under the full $\mathrm{SO}(5)$ symmetry. The quantum numbers of the $\mathcal{O}_{L, R}$ operators determine the representations of the fields that can be directly coupled to the elementary fermions. The fundamental representation of $\mathrm{SO}(5)$ decomposes under $\mathrm{SO}(4)$ as $\mathbf{5}=\mathbf{4}+\mathbf{1}$. For this reason we include in our theory two composite fermion multiplets corresponding to representations $\mathbf{4}_{\mathbf{2} / \mathbf{3}}$ and $\mathbf{1}_{\mathbf{2} / \mathbf{3}}$ of $\mathrm{SO}(4) \times \mathrm{U}(1)_{X}$, which we denote by $\psi_{4}$ and $\psi_{1}$ respectively.

In order to estimate the size of the coefficients of the various terms in the effective Lagrangian we need to use a suitable power-counting rule. Following the approach of refs. $[19,40]$ we adopt the following formula

$$
\mathcal{L}=\sum \frac{m_{*}^{4}}{g_{*}^{2}}\left(\frac{y \psi_{e l}}{m_{*}^{3 / 2}}\right)^{n_{e l}}\left(\frac{g_{*} \Psi}{m_{*}^{3 / 2}}\right)^{n_{c o}}\left(\frac{\partial}{m_{*}}\right)^{n_{d}}\left(\frac{\Pi}{f}\right)^{n_{\pi}}\left(\frac{g A_{\mu}}{m_{*}}\right)^{n_{A}},
$$

where $\psi_{e l}$ generically denotes the elementary fields $q_{L}$ or $t_{R}$, while $\Psi$ denotes the composite fermions. Notice that each insertion of an elementary fermion is accompanied by a corresponding factor of the elementary-composite mixing $y$. We assume that the rule in eq. (2.5) has only two exceptions [40]. ${ }^{1}$ The first one is the kinetic term of the elementary fermions, which we set to be canonical. This is justified by the fact that the elementary fermions are external with respect to the strong dynamics and their kinetic term is set by the UV theory. The second exception is the mass of the fermion resonances included in our low-energy description, which we assume to be smaller than the cut-off $m_{*}$. This is needed in order to write an effective theory in which only a few resonances are present, while the other ones, at the scale $m_{*}$, are integrated out.

The full effective Lagrangian can be split into three pieces which correspond to the terms containing only composite states, the ones containing only elementary fields and the elementary-composite mixings:

$$
\mathcal{L}=\mathcal{L}_{\text {comp }}+\mathcal{L}_{\text {elem }}+\mathcal{L}_{\text {mixing }}
$$

The leading order Lagrangian for the composite fermions is given by

$$
\mathcal{L}_{\text {comp }}=i \bar{\psi}_{4} \not D \psi_{4}+i \bar{\psi}_{1} \not D \psi_{1}-m_{4} \bar{\psi}_{4} \psi_{4}-m_{1} \bar{\psi}_{1} \psi_{1}+\left(i c \bar{\psi}_{4}^{i} \gamma^{\mu} d_{\mu}^{i} \psi_{1}+\text { h.c. }\right)+\frac{1}{f^{2}}(\bar{\psi} \psi)^{2}
$$

\footnotetext{
${ }^{1}$ Notice that the power-counting rule can also be violated in the presence of sum rules which forbid the generation of some operators.
} 

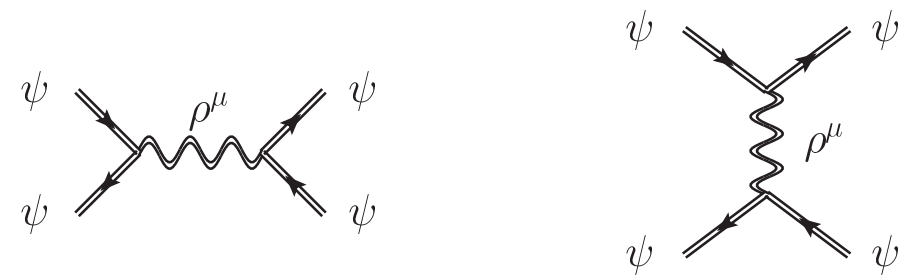

Figure 1. Structure of the Feynman diagrams that generate 4-fermions operator through the exchange of heavy gauge resonances. In the diagrams we represent the composite resonances with a double line.

where the index $i$ labels components of the $\mathrm{SO}(4) 4$-plets. Notice that the covariant derivative of the $\psi_{4}$ field contains, in addition to the usual derivative and to the coupling to the $\mathrm{U}(1)_{Y}$ gauge boson $B_{\mu}$, the CCWZ $e_{\mu}$ symbol: $D_{\mu} \psi_{4}=\left(\partial_{\mu}-2 / 3 i g^{\prime} B_{\mu}+i e_{\mu}\right) \psi_{4}$. The presence of the $e_{\mu}$ term is essential to restore the full $\mathrm{SO}(5)$ invariance of the Lagrangian and gives rise to non-linear derivative couplings between the 4-plet components and the Goldstones. In addition to the usual kinetic and mass terms we can also write an additional term using the CCWZ $d_{\mu}$ symbol. This operator induces some interactions between the 4-plet and the singlet mediated by the gauge fields and by the Goldstones. In general two independent terms with the $d_{\mu}$ symbol can be present, one for the left-handed and one for the right-handed composite fermions. For simplicity, however, we assumed that the strong sector is invariant under parity, which forces the two operators to have the same coefficient.

Finally we denote collectively by $(\bar{\psi} \psi)^{2} / f^{2}$ possible contact interactions with 4 composite fermions. In spite of having dimension 6 these operators are not suppressed by the cut-off $m_{*}$, instead, their natural coefficient is of order $1 / f^{2}$. Operators of this kind are typically generated by the exchange of heavy vector or scalar resonances (see diagrams in figure 1). The suppression due to the propagator of the heavy boson is compensated by the large coupling, $g_{*} \simeq m_{*} / f$, thus explaining the order $1 / f^{2}$ coefficient.

The Lagrangian involving the elementary fields includes the usual canonical kinetic terms

$$
\mathcal{L}_{\text {elem }}=i \bar{q}_{L} \not D q_{L}+i \bar{t}_{R} \not D t_{R}
$$

and the elementary-composite mixing

$$
\begin{aligned}
\mathcal{L}_{\text {mixing }}= & y_{L 4} f\left(\bar{q}_{L}^{\mathbf{5}}\right)^{I} U_{I i} \psi_{4}^{i}+y_{L 1} f\left(\bar{q}_{L}^{\mathbf{5}}\right)^{I} U_{I 5} \psi_{1}+\text { h.c. } \\
& +y_{R 4} f\left(\bar{t}_{R}^{\mathbf{5}}\right)^{I} U_{I i} \psi_{4}^{i}+y_{R 1} f\left(\bar{t}_{R}^{\mathbf{5}}\right)^{I} U_{I 5} \psi_{1}+\text { h.c. }
\end{aligned}
$$

where $q_{L}^{\mathbf{5}}$ and $t_{R}^{\mathbf{5}}$ denote the embedding of the elementary fermions in an incomplete $\mathbf{5}$ of $\mathrm{SO}(5)$, namely

$$
q_{L}^{\mathbf{5}}=\frac{1}{\sqrt{2}}\left(\begin{array}{c}
i b_{L} \\
b_{L} \\
i t_{L} \\
-t_{L} \\
0
\end{array}\right), \quad t_{R}^{\mathbf{5}}=\left(\begin{array}{c}
0 \\
0 \\
0 \\
0 \\
t_{R}
\end{array}\right)
$$


and $U$ is the Goldstone matrix defined in eq. (2.3). The form of the elementary-composite mixings is dictated by the $\mathrm{SO}(5)$ symmetry. The assumption of partial compositeness encoded in eq. (2.1) tells us that the elementary fields are mixed with operators that transform in a linear representation of $\mathrm{SO}(5)$. The $\psi_{4}$ and $\psi_{1}$ CCWZ fields, instead, transform nonlinearly under the global symmetry, so they can not be directly mixed with the elementary fields. To write down a mixing term we thus need to compensate for the non-linear transformation and this can be done by multiplying the CCWZ fields by the Goldstone matrix.

Notice that the coefficients that appear in our effective Lagrangian are in general complex. By means of chiral rotations of the elementary and composite fields one can remove only 3 complex phases, thus some parameters are still complex. In order to simplify the analysis we assume that our Lagrangian is invariant under CP [40]. Under this hypothesis all the parameters in the Lagrangian in eqs. (2.7) and (2.9) are real. $^{2}$

\section{General analysis of the EW parameters}

In this section we provide a general analysis of the new physics corrections to the EW observables, in particular we will focus on the oblique parameters, $\widehat{S}$ and $\widehat{T}$, and on the $Z \bar{b}_{L} b_{L}$ coupling. As we will see, several effects can generate distortions of this parameters and it is important to carefully study all of them. The primary aim of this section is to estimate the size of the various corrections and to determine which observables can be reliably computed in our low-energy effective approach.

\subsection{The oblique parameters}

We start our analysis by considering the oblique EW parameters, $\widehat{S}$ and $\widehat{T}$, [68, 69] that encode the corrections to the two point functions of the EW gauge bosons. The contributions to the oblique parameters come from three main effects: the Goldstone nature of the Higgs, the presence of vector resonances and the presence of fermionic resonances.

The first effect is related to the non-linear Higgs dynamics which induces a modification of the Higgs couplings with the EW gauge bosons. This distortion is present in any composite-Higgs model and is fully determined by the symmetry breaking pattern that gives rise to the Goldstones, in our case $\mathrm{SO}(5) / \mathrm{SO}(4)$. In particular the leading logarithmicallyenhanced contribution is universal and is completely fixed by the IR dynamics [47]. As we will see, while the contribution to $\widehat{S}$ is small, the effect on $\widehat{T}$ is sizable and, without further corrections, would lead to very stringent bounds on the Higgs compositeness scale $f$.

The second source of corrections is the presence of EW gauge resonances. In our effective Lagrangian approach the gauge resonances have been integrated out, thus this corrections arise as a purely UV effect. The most important contribution is generated at tree level due to the mixing of the composite resonances with the elementary gauge bosons and it gives a sizable correction to the $\widehat{S}$ parameter.

Finally the third class of contributions comes from loop effects induced by the composite fermions. This is the class of contributions we will be mainly interested in in the

\footnotetext{
${ }^{2}$ The CP invariance fixes the coefficient of the $d_{\mu}$ symbol term to be purely imaginary. Thus our parameter $c$ is real.
} 


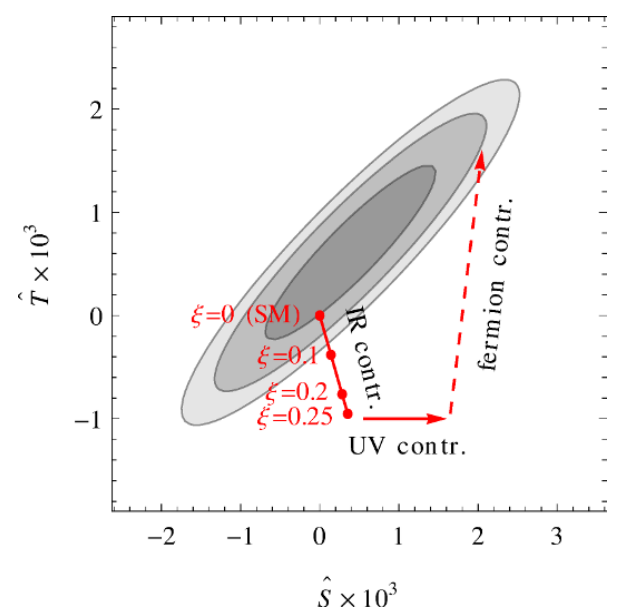

Figure 2. Constraints on the oblique EW parameters $\widehat{S}$ and $\widehat{T}$ [70]. The gray ellipses correspond to the $68 \%, 95 \%$ and $99 \%$ confidence level contours for $m_{h}=126 \mathrm{GeV}$ and $m_{t}=173 \mathrm{GeV}$. The red lines show the contributions that arise in composite Higgs models as explained in the main text. The IR contribution corresponds to the corrections due to non-linear Higgs dynamics, approximately given in eqs. (3.2) and (3.7), and is obtained fixing $m_{*} \sim 3 \mathrm{TeV}$. The UV contribution is due to the EW gauge resonances (see eq. (3.1)).

present analysis. As we will see, these corrections are typically large and including them is essential in order to obtain a reliable fit of the EW parameters. Although these effects have been already considered in the literature, most of the previous analyses did not take into account the full non-linear structure of the composite Higgs Lagrangian. Our analysis will show that the non-linearities are relevant and their inclusion can significantly affect the result and lead to new important effects.

The $\widehat{\boldsymbol{S}}$ parameter. At tree level the $\widehat{S}$ parameter receives a correction due to the mixing of the elementary gauge fields with the composite vector bosons. An estimate of this correction is given by [19]

$$
\Delta \widehat{S} \simeq \frac{g^{2}}{g_{*}^{2}} \xi \simeq \frac{m_{w}^{2}}{m_{*}^{2}}
$$

The UV dynamics can lead to deviations with respect to the above formula. However those deviations are typically small and eq. (3.1) is usually in good agreement with the predictions of explicit models. Assuming that the correction in eq. (3.1) is the dominant contribution to $\widehat{S}$ (or at least that the other contributions to $\widehat{S}$ are positive), a rather strong upper bound on the mass of the EW gauge resonances is found, $m_{*} \gtrsim 2 \mathrm{TeV}$ (see the fit of the oblique parameters in figure 2).

The other contributions to the $\widehat{S}$ parameter arise at loop level due to the non-linear Higgs dynamics and to the presence of fermion resonances. The leading contribution due to the non-linear Higgs dynamics is given by [47]

$$
\Delta \widehat{S}=\frac{g^{2}}{192 \pi^{2}} \xi \log \left(\frac{m_{*}^{2}}{m_{h}^{2}}\right) \simeq 1.4 \cdot 10^{-3} \xi
$$


where $g$ denotes the $\mathrm{SM} \mathrm{SU}(2)_{L}$ gauge coupling. In the above formulae we identified the cut-off with the mass scale of the EW gauge resonances and we chose $m_{*} \sim 3 \mathrm{TeV}$ and $m_{h}=126 \mathrm{GeV}$ to derive the numerical estimate.

The contribution in eq. (3.2) arises from one-loop diagrams with gauge bosons and Goldstone virtual states. The diagrams contributing to $\widehat{S}$ are superficially logarithmically divergent. However, in the SM the logaritmic divergence exactly cancels due to the physical Higgs contribution. This is no longer true when the Higgs couplings are modified and in composite Higgs models a residual logarithmic dependence on the cut-off scale is present. ${ }^{3}$ As can be seen from the numerical estimate the contribution in eq. (3.2) is much smaller than the absolute bounds on $\widehat{S}$ (compare figure 2) and is typically negligible.

Let us finally consider the contribution due to loops of fermionic resonances. The general expression for the corrections to $\widehat{S}$ due to an arbitrary set of new vector-like fermion multiplets has been derived in ref. [72]. The final formula contains a divergent contribution to $\widehat{S}$ given by

$$
\Delta \widehat{S}_{\mathrm{ferm}}^{\mathrm{div}}=\frac{N_{c} g^{2}}{96 \pi^{2}} \operatorname{Tr}\left[U_{L}^{\dagger} Y_{L}+U_{R}^{\dagger} Y_{R}\right] \log \left(m_{*}^{2}\right),
$$

where $U_{L, R}$ and $Y_{L, R}$ are the matrices of the couplings of left- and right-handed fermions to the $W_{\mu}^{3}$ and to the $B_{\mu}$ gauge bosons respectively and $N_{c}$ is the number of QCD colors. In a renormalizable theory in which the couplings of the gauge bosons to the fermions are just given by the usual covariant derivatives it is easy to see that the trace appearing in eq. (3.3) vanishes, so that no logarithmically divergent contribution to $\widehat{S}$ is present. ${ }^{4}$ This is no longer true when the Higgs is a Goldstone boson. In this case higher order interactions of the gauge bosons mediated by the Higgs are present in the Lagrangian. Interactions of this kind are contained in the $e_{\mu}$ term in the covariant derivative of the composite 4-plet $\psi_{4}$ and in the $d_{\mu}$-symbol term. After EWSB a distortion of the gauge couplings to the fermions is induced by these operators and a logarithmically divergent contribution to $\widehat{S}$ is generated. The presence of a logarithmically enhanced contribution can be also understood in simple terms as a running of the operators related to the $\widehat{S}$ parameter. We postpone a discussion of this aspect to the end of this subsection.

The logarithmically divergent correction can be straightforwardly computed:

$$
\Delta \widehat{S}_{\mathrm{ferm}}^{\mathrm{div}}=\frac{g^{2}}{8 \pi^{2}}\left(1-2 c^{2}\right) \xi \log \left(\frac{m_{*}^{2}}{m_{4}^{2}}\right) .
$$

It is important to notice that this contribution is there only if at least one $\mathrm{SO}(4) 4$-plet is present in the effective theory. In fact, as we said, the only terms in the effective Lagrangian that can lead to relevant distortions of the gauge couplings are the 4-plet kinetic term and the $d_{\mu}$-symbol term, which are clearly absent if only singlets are present. The connection of the divergence with the 4-plets justifies the identification of the argument of the logarithm in

\footnotetext{
${ }^{3} \mathrm{~A}$ more detailed analysis of the corrections to the $\widehat{S}$ parameter related to the Goldstone nature of the Higgs has been presented in ref. [71].

${ }^{4}$ To prove this one can notice that the sum of the $W_{\mu}^{3}$ couplings to the fermions in each $\mathrm{SU}(2)_{L}$ multiplet is zero. After EWSB the gauge couplings of the fermion mass eigenstates are obtained by unitary rotations of the initial coupling matrices. These rotation clearly cancel out in the trace in eq. (3.3), so that the divergent term vanishes.
} 


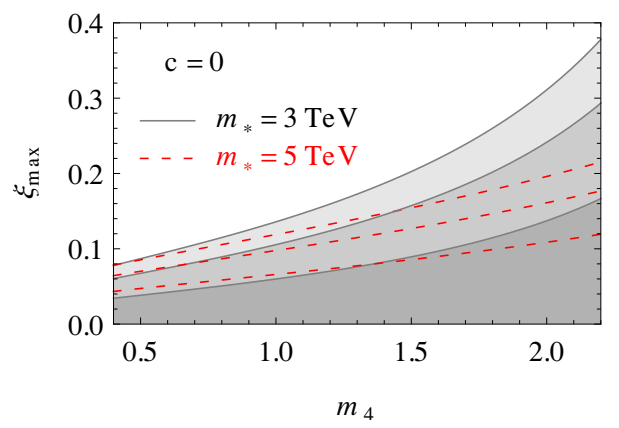

Figure 3. Upper bounds on $\xi$ in the 2-site model $(c=0)$ as a function of the 4-plet mass parameter $m_{4}$ for different values of the cut-off $m_{*}$. The results have been obtained by considering the shift in $\widehat{S}$ given in eqs. (3.1), (3.2) and (3.4) and by marginalizing on $\widehat{T}$. The shaded regions correspond to the points compatible with the constraints at the $68 \%, 95 \%$ and $99 \%$ confidence level for $m_{*}=3 \mathrm{TeV}$. The dashed red curves show how the bounds are modified for $m_{*}=5 \mathrm{TeV}$.

eq. (3.4) with the ratio $m_{*}^{2} / m_{4}^{2}$. It is also remarkable the fact that the correction in eq. (3.4) is independent of the elementary-composite mixings $y_{L, R}$. This implies that any $\mathrm{SO}(4) 4-$ plet below the cut-off of the effective theory would contribute to $\widehat{S}$ with a similar shift. ${ }^{5}$

Notice that, in order to derive the result in eq. (3.4), we assumed that the logarithmic divergence due to the fermion loops is regulated at the cut-off scale $m_{*}$. This is expected to happen as a consequence of the presence of EW gauge resonances with a mass of order $m_{*}$. Peculiar UV dynamics, however, could modify this picture and push up the scale at which the divergence is regulated, resulting in a larger contribution to $\widehat{S}$.

Another interesting property of the divergent contribution to $\widehat{S}$ is the fact that it vanishes if $c^{2}=1 / 2$. As we will see later on, this choice of the parameter $c$ implies the presence of an extra symmetry in the effective Lagrangian which protects the EW observables.

The logarithmic contribution to $\widehat{S}$ in eq. (3.4) is sizable if $c^{2}$ is not too close to $1 / 2$ and is typically much larger than the corresponding effect due to the Higgs non-linearities (eq. (3.2)). The correction due to fermion loops can even be comparable with the tree-level contribution estimated in eq. (3.1) if the strong coupling $g_{*}$ is large, $g_{*} \gtrsim 5$. From the point of view of our effective approach, the coefficient $c$ is just a free parameter, thus in principle the divergent fermion contribution can have an arbitrary sign. In particular for $c^{2}>1 / 2$ a sizable negative shift in $\widehat{S}$ would be possible, which could improve the agreement with the EW precision measurements (see figure 2).

It is important to notice that in explicit models that provide a partial UV completion of our effective theory the value of $c$ is typically fixed. A possible extension of our effective Lagrangian is given by the 2 -site model proposed in refs. $[13,66]$. In this model $c=0$, so that a sizable positive shift in $\widehat{S}$ seems unavoidable if a relatively light 4-plet is present. For example for $m_{4} \simeq 700 \mathrm{GeV}$ and $m_{*} \simeq 3 \mathrm{TeV}$ a tight upper bound, $\xi \lesssim 0.1$, is obtained if we marginalize on $\widehat{T}$. The limits on the compositeness scale as a function of the 4 -plet mass taking into account only the constraints on the $\widehat{S}$ parameter are shown in figure 3 .

\footnotetext{
${ }^{5}$ Resonances in larger $\mathrm{SO}(4)$ multiplets also lead to divergent contributions. For instance, states in the 9 lead to a contribution 6 times larger than the one in eq. (3.4).
} 
Notice that the bounds become typically stronger if the cut-off scale increases. This is due to the fact that the logarithmically enhanced fermion contribution in eq. (3.4) grows at larger $m_{*}$ and dominates over the tree-level correction in eq. (3.1) which instead decreases when the gauge resonances become heavier.

The 2-site realization of the composite models allows us also to find a connection between the fermion corrections to $\widehat{S}$ and the dynamics of the gauge resonances. In fact it turns out that the diagrams that give rise to the divergence in $\widehat{S}$ are closely related to the ones that determine the running of the gauge resonance coupling $g_{*}$. The divergent contribution to $\widehat{S}$ in this picture arises from the distortion of the mixing between the elementary and the composite gauge fields after EWSB.

A fermion contribution to $\widehat{S}$ similar to the one we found is in principle present also in the extra-dimensional realization of the composite Higgs scenario. The corrections to the oblique EW parameters due to fermion loops in this class of theories have been considered in the literature $[65,73,74]$, however no divergent or enhanced contribution was noticed. It is probable however that a contribution of this kind was overlooked because of its peculiar origin. Similarly to what happens in the 2-site model, in extra dimensions the divergence in $\widehat{S}$ derives from the mixing of the gauge zero-modes with the gauge resonances after EWSB. In the literature the computation of $\widehat{S}$ has been made neglecting this mixing, thus the divergent contribution was not found.

Notice that, in addition to the divergent contributions, which explicitly depend on the cut-off, large finite contributions can also arise from the UV dynamics of the theory. We can estimate the one-loop UV contributions as

$$
\Delta \widehat{S} \sim \frac{g^{2}}{16 \pi^{2}} \xi \simeq 3 \cdot 10^{-3} \xi
$$

It is easy to see that these effects can in principle be sizable and could significantly change the fit to the EW data. The estimate in eq. (3.5) should be considered as a lower bound on the size of the UV corrections, valid if no accidental cancellations are present. Larger corrections to $\widehat{S}$ are possible in the presence of some peculiar UV dynamics, these however can not be predicted within our effective approach. We will see an explicit example of non-decoupling effects in subsection 4.1.

The corrections to $\widehat{\boldsymbol{S}}$ as a running effect. We can understand in simple terms the origin of the large logarithmically enhanced contributions to the $\widehat{S}$ parameter with an operator approach. In the effective theory the corrections to the $\widehat{S}$ parameter are induced by two dimension- 6 operators [19]:

$$
\mathcal{O}_{W}=i\left(H^{\dagger} \sigma^{i} \overleftrightarrow{D^{\mu}} H\right)\left(D^{\nu} W_{\mu \nu}\right)^{i} \quad \text { and } \quad \mathcal{O}_{B}=i\left(H^{\dagger} \overleftrightarrow{D^{\mu}} H\right)\left(D^{\nu} B_{\mu \nu}\right)
$$

where $H$ denotes the usual Higgs doublet and $H^{\dagger} \overleftrightarrow{D_{\mu}} H$ is the derivative $H^{\dagger}\left(D_{\mu} H\right)-$ $\left(D_{\mu} H\right)^{\dagger} H$.

The corrections to the $\mathcal{O}_{W, B}$ operators can be connected to the diagrams with two external Higgs states and one gauge field. In a renormalizable theory with only standard Yukawa Higgs couplings to the fermions the corrections from heavy resonances loops come 


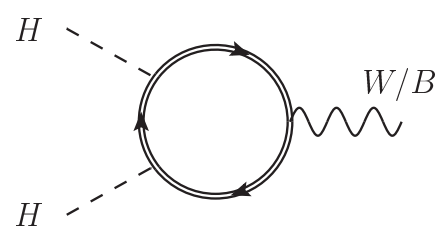

(a)

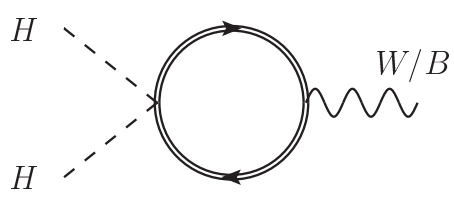

(b)

Figure 4. Diagrams with resonance loops that can contribute to the $\mathcal{O}_{W, B}$ operators.

from the $(a)$ diagrams in figure 4 . By noticing that the $\mathcal{O}_{W, B}$ operators contain three powers of the external momenta it is easy to realize that these diagrams are always finite.

In a theory with a non-linear Higgs dynamics the situation is instead drastically different. In this case non-renormalizable contact interactions with two Higgses and two composite fermions are present. In particular the $e_{\mu}$ symbol in the kinetic term of the composite 4-plets induces a non-renormalizable interaction $i\left(\vec{\Pi}^{t} t^{a} \partial_{\mu} \vec{\Pi}\right)\left(\bar{\psi}_{4} \gamma^{\mu} \psi_{4}\right.$ ) (see the explicit results in appendix A). This non-linear vertex, together with the usual gauge interactions, gives rise to the new class of diagrams denoted by $(b)$ in figure 4 . These diagrams are logarithmically divergent and induce a corresponding running of the $\mathcal{O}_{W, B}$ operators leading to an enhanced contribution to $\widehat{S}$. This running effect generates the $c$-independent term in the correction to $\widehat{S}$ (see eq. (3.4)). ${ }^{6}$

Non-renormalizable Higgs interactions are also generated by the $d_{\mu}$ symbol terms. In particular it gives rise to a new vertex of the form $\left(\partial_{\mu} \Pi^{i}\right) \bar{\psi}_{4}^{i} \gamma^{\mu} \psi_{1}+$ h.c.. This vertex induces a logarithmically divergent contribution to $\mathcal{O}_{W, B}$ through diagrams analogous to the type (a) shown in figure 4 . The related contribution to the $\widehat{S}$ parameter corresponds to the term proportional to $c^{2}$ in eq. (3.4).

It is interesting to notice that similar contributions to the $\widehat{S}$ parameter are also present in technicolor models but originated from the non-linear dynamics not of the whole Higgs doublet, as in our case, but only of the Goldstones associated to the spontaneous breaking $\mathrm{SU}(2)_{L} \times \mathrm{SU}(2)_{R} \rightarrow \mathrm{SU}(2)_{V}[75,76]$.

Before concluding the discussion on $\widehat{S}$ we want to comment on the relation between our results and the ones of refs. [77, 78]. In refs. [77, 78] an effective approach was used in which only the SM fields are retained and all the composite resonances are integrated out. In this framework it was shown that two effective operators $\mathcal{O}_{H q}=i\left(\bar{q}_{L} \gamma^{\mu} q_{L}\right)\left(H^{\dagger} \overleftrightarrow{D_{\mu}} H\right)$ and $\mathcal{O}_{H q}^{\prime}=i\left(\bar{q}_{L} \gamma^{\mu} \sigma^{i} q_{L}\right)\left(H^{\dagger} \sigma^{i} \overleftrightarrow{D_{\mu}} H\right)$ induce a logarithmic running for $\widehat{S}$ between the top mass, $m_{t}$ and the energy scale at which the effective operators are generated, $m$. Differently from refs. $[77,78]$, in our approach the resonances are included in the effective theory and the effective operators $\mathcal{O}_{H q}$ and $\mathcal{O}_{H q}^{\prime}$ are not present directly in our Lagrangian. At low energy, however, they are generated through the exchange of resonances of mass $m$ with a coefficient $y^{2} / \mathrm{m}^{2}$. From the previous discussion it is easy to understand that in our approach the

\footnotetext{
${ }^{6}$ Notice that the diagrams with the new non-linear Higgs vertex can in principle contribute also to two other dimension-6 operators, $\mathcal{O}_{H W}=i\left(D^{\mu} H\right)^{\dagger} \sigma^{i}\left(D^{\nu} H\right) W_{\mu \nu}^{i}$ and $\mathcal{O}_{H B}=i\left(D^{\mu} H\right)^{\dagger}\left(D^{\nu} H\right) B_{\mu \nu}$. Differently from $\mathcal{O}_{W, B}$, these two operators do not contribute to $\widehat{S}$ and are not minimally coupled [19]. With an explicit computation we found that the logarithmically divergent diagrams only generate a running of the minimally coupled operators $\mathcal{O}_{W, B}$ and not of $\mathcal{O}_{H W, H B}$.
} 


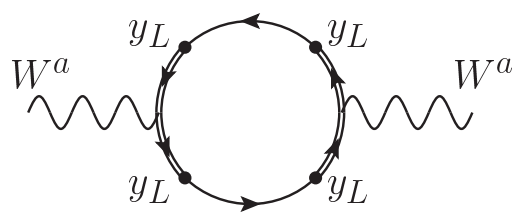

Figure 5. Schematic structure of a fermion loop diagram contributing to the $\widehat{T}$ parameter at leading order in the $y$ expansion.

logarithmically divergent corrections to $\widehat{S}$ found in refs. [77, 78] do not appear as real divergences but rather correspond to corrections that scale as $y^{2} / m^{2} \log \left(m^{2} / m_{t}^{2}\right)$. Terms of this form can be recognized, for example, in the explicit analytic result for $\widehat{S}$ given in eq. (4.2). ${ }^{7}$

The $\widehat{\boldsymbol{T}}$ parameter. We can now analyze the corrections to the $\widehat{T}$ parameter. Thanks to the custodial symmetry $\widehat{T}$ does not receive correction at tree level and the only contributions come at loop level from diagrams with insertions of the operators that break the custodial symmetry. In our effective Lagrangian this breaking is induced by the weak gauging of the hypercharge $\mathrm{U}(1)_{Y}$ with coupling $g^{\prime}$ and by the mixings $y_{L 4,1}$ of the $q_{L}$ elementary doublet with the composite fermions.

The main correction due to the hypercharge coupling breaking comes from the IR contribution associated to the Goldstone nature of the Higgs. This effect is analogous to the one we already discussed for the $\widehat{S}$ parameter. The leading logarithmically enhanced contribution is given by [47]

$$
\Delta \widehat{T}=-\frac{3 g^{\prime 2}}{64 \pi^{2}} \xi \log \left(\frac{m_{*}^{2}}{m_{h}^{2}}\right) \simeq-3.8 \cdot 10^{-3} \xi .
$$

Differently from the analogous contribution to $\widehat{S}$, which was negligible due to accidental suppression factors, the contribution in eq. (3.7) gives a sizable correction to $\widehat{T}$. In particular, if we assume that this is the dominant correction to $\widehat{T}$ and that the shift in $\widehat{S}$ is non negative, a very stringent bound on $\xi$ is obtained, $\xi \lesssim 0.1$ (see figure 2 ). ${ }^{8}$

The second correction comes from fermion loops. As already noticed, in order to induce a contribution to $\widehat{T}$ the corresponding diagrams must contain some insertions of the symmetry breaking couplings $y_{L 4,1}$. Under $\mathrm{SU}(2)_{L} \times \mathrm{SU}(2)_{R}$ the $y_{L 4,1}$ mixings transform in the $(\mathbf{1}, \mathbf{2})$ representation, thus at least 4 insertions are needed to generate a shift in $\widehat{T}$ [19]. This minimal number of insertions guarantees that the fermion one-loop corrections to $\widehat{T}$ are finite. A typical diagram contributing at leading order in the $y$ expansion is shown in figure 5 .

It is straightforward to estimate the corrections to $\widehat{T}$ at leading order in the elementarycomposite mixing [19]:

$$
\Delta \widehat{T} \simeq \frac{N_{c}}{16 \pi^{2}} \frac{y_{L}^{4} f^{2}}{m^{2}} \xi
$$

\footnotetext{
${ }^{7}$ Notice that other effective operators with the structure $\mathcal{O}_{t}=H^{\dagger} H\left(\bar{q}_{L} H^{c} t_{R}\right)$ do not generate a running for $\widehat{S}[77]$.

${ }^{8} \mathrm{~A}$ similar bound has been derived in ref. [49], where the phenomenological impact of the IR corrections to $\widehat{S}$ and $\widehat{T}$ on the fit of the Higgs couplings has been analyzed.
} 
where we denoted by $m$ the mass scale of the lightest top partners in our effective Lagrangian. To get a quantitative estimate we can extract the value of the $y_{L}$ mixing from the top mass. If we assume that the elementary-composite mixings have comparable sizes, $y_{L 4} \simeq y_{L 1} \simeq y_{R 4} \simeq y_{R 1} \simeq y$, the top Yukawa can be estimated as $y_{t} \simeq y^{2} f / m$. By using this expression we get the estimate

$$
\Delta \widehat{T} \simeq \frac{N_{c}}{16 \pi^{2}} y_{t}^{2} \xi \simeq 2 \cdot 10^{-2} \xi .
$$

Notice that this contribution is usually dominant with respect to the one given in eq. (3.7). Moreover, as we will see in the next section with an explicit calculation, the sign of the fermion contribution can be positive, so that it can compensate the negative shift in eq. (3.7). Notice that, if $\widehat{S}$ is not negative, a positive correction to $\widehat{T}$ from the fermion loops is essential in order to satisfy the EW constraints as can be clearly seen from the bound in figure 2 .

Notice that the finiteness of the fermion loop contribution to $\widehat{T}$ implies that the correction coming from the lightest resonances is dominant with respect to the one coming from heavier states. The contribution due to the UV dynamics can be estimated as [19]

$$
\Delta \widehat{T} \simeq \frac{N_{c}}{16 \pi^{2}} \frac{y_{L}^{4}}{g_{*}^{2}} \xi
$$

This contribution is suppressed with respect to the one in eq. (3.8) by a factor $m^{2} / m_{*}^{2}$. This shows that $\widehat{T}$ can be predicted in a robust way using our effective field theory approach.

\subsection{The $Z \bar{b}_{L} b_{L}$ vertex}

Another observable that can be used to constrain the parameter space of new physics models is the $Z$ boson coupling to the left-handed bottom quark. We define the $Z$ interactions with the bottom by the formula

$$
\mathcal{L}^{Z}=\frac{g}{c_{w}} Z_{\mu} \bar{b} \gamma^{\mu}\left[\left(g_{b_{L}}^{\mathrm{SM}}+\delta g_{b_{L}}\right) P_{L}+\left(g_{b_{R}}^{\mathrm{SM}}+\delta g_{b_{R}}\right) P_{R}\right] b
$$

where $g^{\mathrm{SM}}$ denotes the SM couplings (including the loop corrections), $\delta g$ denotes the corrections due to new physics and $P_{L, R}$ are the left and right projectors. In the following we will denote by $s_{w}$ and $c_{w}$ the sine and cosine of the weak mixing angle. The SM tree-level values for the couplings are

$$
g_{b_{L}}^{\mathrm{SM}, \text { tree }}=-\frac{1}{2}+\frac{1}{3} s_{w}^{2}, \quad g_{b_{R}}^{\mathrm{SM}, \text { tree }}=\frac{1}{3} s_{w}^{2},
$$

and the one-loop corrections (computed in the limit $g \rightarrow 0$ ) are

$$
g_{b_{L}}^{\mathrm{SM}, \text { loop }}=\frac{m_{t}^{2}}{16 \pi^{2} v^{2}}, \quad g_{b_{R}}^{\mathrm{SM}, \text { loop }}=0
$$

As can be seen from the current bounds shown in figure 6 , the deviation of the $Z \bar{b}_{L} b_{L}$ coupling are constrained to be at the level $3 \cdot 10^{-3}$, while the bounds on the coupling with the right-handed bottom component are one order of magnitude less stringent. In composite 


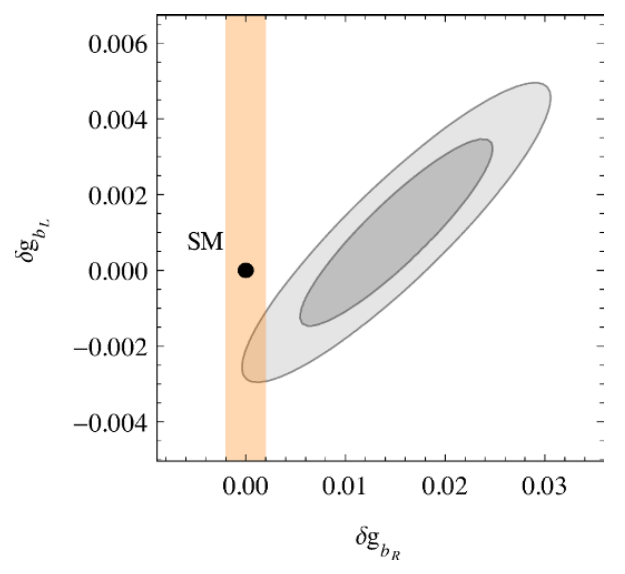

Figure 6. Constraints on the corrections to the $Z$ boson couplings to the bottom quark. The ellipses show the exclusion contours at $68 \%$ and $95 \%$ confidence level $[79,80]$. The vertical band shows the expected size of the corrections to the $g_{b_{R}}$ coupling.

models the corrections to the $g_{b_{R}}$ coupling are typically small, at most of the same order of the deviations in $g_{b_{L}}$. If we impose the constraint $\left|\delta g_{b_{R}}\right| \lesssim f e w \cdot 10^{-3}$, a negative value for $\delta g_{b_{L}}$ of order $-2 \cdot 10^{-3}$ is preferred, while a positive shift worsens the fit with respect to the SM. The region favored by the current fit in the $\left(\delta g_{b_{L}}, \delta g_{b_{R}}\right)$ plane is shown in figure 6 and corresponds to the intersection of the gray ellipses with the vertical band.

\section{Tree-level corrections}

Let us now analyze the new physics corrections that arise in our scenario. The presence of an automatic $P_{L R}$ symmetry in the composite sector and the fact that the elementary $b_{L}$ state is invariant under this symmetry implies the absence of tree-level corrections to the $Z \bar{b}_{L} b_{L}$ vertex at zero momentum $[81,82]$. The tree-level corrections induced at non-zero momentum are related to operators of the form $D_{\mu} F^{\mu \nu} \bar{q}_{L} \gamma_{\nu} q_{L}$ and their size can be estimated as

$$
\frac{\delta g_{b_{L}}}{g_{b_{L}}^{\mathrm{SM}}} \sim \frac{y_{L}^{2} f^{2}}{m^{2}} \frac{m_{z}^{2}}{m_{*}^{2}} \simeq 8 \cdot 10^{-4} \frac{f}{m}\left(\frac{4 \pi}{g_{*}}\right)^{2} \xi,
$$

where $m$ is the mass scale of the composite fields mixed with the bottom, which in our scenario correspond to the charge $-1 / 3$ state inside the 4 -plet $\psi_{4}$.

Notice that in our effective Lagrangian we did not include an elementary $b_{R}$ state. For this reason the bottom is massless in our theory. In a more complete scenario a chiral field corresponding to the $b_{R}$ will be present together extra composite fermions that are needed to generate the bottom mass. In this case the elementary $q_{L}$ doublet has additional mixing terms with the new resonances and a tree-level correction to the $Z \bar{b}_{L} b_{L}$ vertex could be generated. For instance this happens in the case in which the additional bottom partners are contained in a 5 of $\mathrm{SO}(5)$ with $\mathrm{U}(1)_{X}$ charge $-1 / 3$. The contribution to the $Z \bar{b}_{L} b_{L}$ vertex coming from these states can be estimated as

$$
\frac{\delta g_{b_{L}}}{g_{b_{L}}^{\mathrm{SM}}} \simeq \frac{\left(y_{L}^{b} f\right)^{2}}{m_{B}^{2}} \xi
$$


where we denoted by $y_{L}^{b}$ the mixing of $q_{L}$ to the new multiplet and by $m_{B}$ the typical mass scale of the new bottom partners. We can relate $y_{L}^{b}$ to the bottom Yukawa by assuming that $y_{L}^{b} \simeq y_{R}^{b}$, in this case $\left(y_{L}^{b}\right)^{2} \simeq\left(y_{R}^{b}\right)^{2} \simeq y_{b} m_{B} / f$. The correction in eq. (3.15) becomes

$$
\frac{\delta g_{b_{L}}}{g_{b_{L}}^{\mathrm{SM}}} \simeq y_{b} \frac{f}{m_{B}} \xi \simeq 2 \cdot 10^{-2} \frac{f}{m_{B}} \xi .
$$

This correction can easily have a size comparable with the current bounds on $\delta g_{b_{L}}$ in the case in which the new bottom partners are relatively light. Of course this correction can be suppressed if we relax the assumption $y_{L}^{b} \simeq y_{R}^{b}$ or if we chose $m_{B} \gg f$.

\section{Corrections from fermion loops}

We can now consider the one-loop contributions to the $Z \bar{b}_{L} b_{L}$ vertex. As a first step we will analyze the degree of divergence of the diagrams contributing to this effect. The degree of divergence can be easily obtained by using the power-counting method explained in ref. [66]. It is straightforward to check that the $Z \bar{b}_{L} b_{L}$ operator at one loop is naively associated to a quadratic divergence. In our set-up, however, the $P_{L R}$ symmetry implies a reduction of the naive degree of divergence. This is an obvious consequence of the fact that a new physics contribution to the $Z \bar{b}_{L} b_{L}$ vertex can be generated only if some powers of the couplings that break the $P_{L R}$ symmetry are inserted in the diagrams. In our Lagrangian only the $y_{L}$ mixings induce a breaking of this symmetry. These mixings correspond to some mass operators, so that each insertion in loop diagrams lowers the degree of divergence by one. ${ }^{9}$ Let us now count how many insertions of the $y_{L}$ mixing are necessary to generate a distortion of the $Z \bar{b}_{L} b_{L}$ vertex. Each external $b_{L}$ is of course associated to a power of $y_{L}$. However, due to the fact that the $b_{L}$ fields are external legs and they are invariant under $P_{L R}$, these insertions do not lead to a breaking of the symmetry. As a consequence at least four insertions of $y_{L}$ are needed to generate a non-vanishing contribution. ${ }^{10}$

If the four $y_{L}$ insertions are all inside the loop the corresponding contribution to the $Z \bar{b}_{L} b_{L}$ vertex is finite. This necessarily happens in the case in which only a singlet is present in the effective theory. Instead, if a 4-plet is also present, two $y_{L}$ insertions can be on the external legs. In this case the two "external" insertions do not influence the degree of divergence and a logarithmically divergent contribution can be present. Examples of diagrams that could lead to this kind of corrections are shown in figure 7 .

In our effective theory a further subtlety is present which partially protects the $Z \bar{b}_{L} b_{L}$ vertex. The structure of the elementary-composite mixings implies the presence of a selection rule that forbids logarithmically divergent corrections coming from a large class of diagrams. As we will see the only diagrams that can lead to a divergent contribution are a subset of the "bubble"-type diagrams (see the diagram on the right of figure 7), so that this kind of correction is necessarily related to the presence of 4 -fermion operators.

\footnotetext{
${ }^{9}$ The $y_{L}$ mixing could in principle appear also in higher-dimensional operators. These operators, which we did not include in our effective Lagrangian, are suppressed by powers of the UV cut-off $m_{*}$ as can be inferred from our power-counting rule in eq. (2.5). For this reason their insertions also lead to a reduction of the degree of divergence in agreement with the power counting expectation.

${ }^{10} \mathrm{~A}$ more rigorous proof of this statement can be obtained by using an operator analysis. For simplicity we do not present this analysis in the main text and postpone it to appendix B.
} 

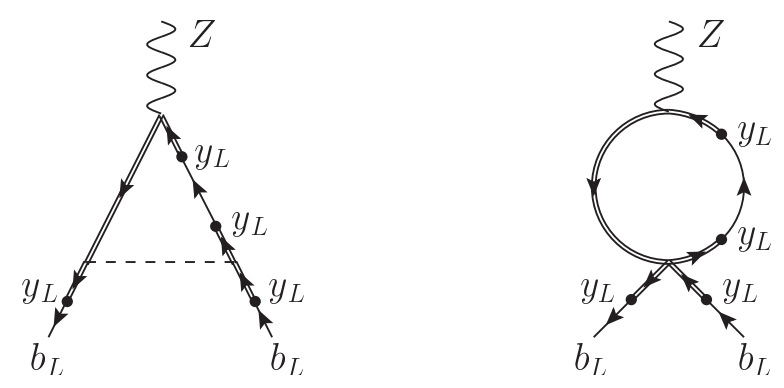

Figure 7. Schematic structure of fermion loop diagrams contributing to the $Z \bar{b}_{L} b_{L}$ vertex with insertions of the $y_{L}$ couplings on the external fermion legs.

To understand the origin of the selection rule we can analyze the "triangle"-type diagrams with $y_{L}$ insertions on the external legs shown on the left of figure 7 . The external $b_{L}$ 's are both mixed with the $B_{L}$ state coming from $\psi_{4}$. In order to generate a divergence the vertices containing a Goldstone boson must also contain a power of the momentum, that is they must be of the type $\partial_{\mu} \phi \bar{\psi}_{L} \gamma^{\mu} \psi_{L}$, where we generically denote by $\phi$ the Goldstone field and by $\psi$ the composite fermions. ${ }^{11}$ The structure of the vertex implies that the composite fermions that enter in the loop must be necessarily left-handed. But the left-handed composite fermions in the leading order Lagrangian mix with the elementary states only through $y_{R}$. As a consequence in order to generate a triangle diagram of this type some $y_{R}$ or some composite mass insertions are needed in addition to the $y_{L}$ mixings and this lowers the degree of divergence making the diagrams finite.

The only diagrams that can give rise to a logarithmic divergence are the "bubble" ones shown on the right of figure 7. They of course crucially depend on the presence of 4fermion operators in the effective Lagrangian. Two types of 4 -fermion vertices can generate a diagram that contributes to $\delta g_{b_{L}}$. The first type of vertex has the form

$$
\mathcal{O}_{L}^{4-\text { ferm }}=\frac{e_{L}}{f^{2}}\left(\bar{B}_{L} \gamma^{\mu} B_{L}\right)\left(\overline{\mathcal{T}}_{L} \gamma_{\mu} \mathcal{T}_{L}\right)
$$

where by $\mathcal{T}$ we denote any composite state with charge $2 / 3$. For shortness in eq. (3.17) we did not specify the color structure which is not relevant for the present discussion. By adapting the previous analysis of the "triangle" diagrams, it is straightforward to show that the "bubble" diagrams with the vertex in eq. (3.17) are also protected by the selection rule, so that they are finite. The second type of 4 -fermion vertex is of the form

$$
\mathcal{O}_{R}^{4-\text { ferm }}=\frac{e_{R}}{f^{2}}\left(\bar{B}_{L} \gamma^{\mu} B_{L}\right)\left(\overline{\mathcal{T}}_{R} \gamma_{\mu} \mathcal{T}_{R}\right)
$$

In this case the selection rule is violated because the $\mathcal{T}_{R}$ fields can clearly mix with the $q_{L}$ doublet through $y_{L}$. This class of vertices, as we will show with an explicit calculation, gives rise to a logarithmically divergent contribution to the $Z \bar{b}_{L} b_{L}$ vertex.

Of course in our effective Lagrangian higher-order mixing terms between the elementary and the composite states can in general be present. An example of such operators is a

\footnotetext{
${ }^{11}$ In our effective Lagrangian vertices of this kind are generated by the $d_{\mu}$ symbol term.
} 
kinetic mixing between the $q_{L}$ doublet and the composite 4-plet: $y_{L} f / m_{*}\left(\bar{q}_{L}^{\mathbf{5}}\right)^{I} U_{I i} \not D \psi_{4 L}^{i}+$ h.c.. A term like this would induce a correction to the $Z \bar{b}_{L} b_{L}$ vertex through diagrams analogous to the "triangle" ones we considered before. Such a diagram would be superficially quadratically divergent (the kinetic higher-order mixing gives an extra power of the momentum). However the coefficient of the kinetic mixing, following our power counting in eq. (2.5), is suppressed by the UV cut-off, $m_{*}$, so that the final contribution is finite. Even though these diagrams can not give a logarithmically divergent contribution, they induce a correction that is not suppressed by powers of the cut-off, thus they can contribute at leading order to the $Z \bar{b}_{L} b_{L}$ vertex.

Notice that the presence of unsuppressed contributions of this kind also implies a nondecoupling of the fermionic resonances. Even if we send the mass of a resonance to the cut-off, it can generate a higher-order effective operator in the low-energy Lagrangian that breaks the selection rule and gives a sizable contribution to the $Z \bar{b}_{L} b_{L}$ vertex. We will discuss an example of this effect in the next section.

The above discussion clearly shows that, even in the absence of logarithmically divergent contributions, the $Z \bar{b}_{L} b_{L}$ vertex is highly sensitive to the UV dynamics of the theory and can be reliably computed in a low-energy effective approach only if the logarithmically divergent contributions dominate or if we assume that the contributions coming from the UV dynamics are (accidentally) suppressed.

To conclude the general analysis of the $Z \bar{b}_{L} b_{L}$ vertex corrections we derive an estimate of the size of the contribution due to the fermion loops. The logarithmically divergent contribution can be estimated as

$$
\frac{\delta g_{b_{L}}}{g_{b_{L}}^{\mathrm{SM}}} \simeq \frac{y_{L}^{2}}{16 \pi^{2}} \frac{y_{L 4}^{2} f^{2}}{m_{4}^{2}+y_{L 4}^{2} f^{2}} \xi \log \left(\frac{m_{*}^{2}}{m_{4}^{2}}\right) .
$$

Notice that we explicitly included a factor $y_{L 4}^{2} f^{2} /\left(m_{4}^{2}+y_{L 4}^{2} f^{2}\right)$, which corresponds to the mixings between the $b_{L}$ and the $B_{L}$ that appears in the external legs of the logarithmically divergent diagrams. Using the relation between $y_{L, R}$ and the top Yukawa we get

$$
\frac{\delta g_{b_{L}}}{g_{b_{L}}^{\mathrm{SM}}} \simeq \frac{y_{t}^{2}}{16 \pi^{2}} \xi \log \left(\frac{m_{*}^{2}}{m_{4}^{2}}\right) \simeq 2 \cdot 10^{-2} \xi,
$$

where for the numerical estimate we set $m_{*} \simeq 3 \mathrm{TeV}$ and $m_{4} \simeq 700 \mathrm{GeV}$. In the case in which the logarithmically divergent contribution is not present or is suppressed the estimate becomes

$$
\frac{\delta g_{b_{L}}}{g_{b_{L}}^{\mathrm{SM}}} \simeq \frac{y_{L}^{2}}{16 \pi^{2}} \frac{y_{L}^{2} f^{2}}{m^{2}} \xi \simeq \frac{y_{t}^{2}}{16 \pi^{2}} \xi \simeq 6 \cdot 10^{-3} \xi,
$$

with $m$ the mass of the lightest top partner.

The corrections in eqs. (3.19) and (3.21) are typically larger than the tree-level contribution generated at non zero momentum given in eq. (3.14). This is especially true if the mass of the resonances is not too small, $m \gtrsim f$, and the strong coupling is large, $g_{*} \gtrsim 5$. The corrections due to the bottom partners estimated in eq. (3.16) can in principle be comparable to the ones coming from fermion loops if the scale of the bottom partner is 
relatively small $m_{B} \sim f$. These corrections crucially depend on the quantum numbers of the bottom partners. In minimal scenarios (bottom partners in the fundamental representation of $\mathrm{SO}(5)$ ) they are positive and some cancellation seems required to pass the present bounds. For simplicity, in our explicit analysis we will neglect both tree-level corrections.

\subsection{Symmetries in the effective Lagrangian}

As we saw in the analysis of the $\widehat{S}$ parameter the divergent contributions coming from fermion loops are finite if the relation $c^{2}=1 / 2$ holds. We want now to study our effective Lagrangian in this case and understand the origin of the protection of the EW parameters. For definiteness we will focus on the case $c=1 / \sqrt{2}$ and we will comment at the end on the other possibility $c=-1 / \sqrt{2}$.

Let us start with the Lagrangian for the composite fields given in eq. (2.7). A straightforward computation shows that the leading order terms in the case $c=1 / \sqrt{2}$ can be simply rewritten as

$$
\left.\mathcal{L}_{\text {comp }}^{c=1 / \sqrt{2}}=i \overline{(} \Psi U^{\dagger}\right) \gamma^{\mu}\left(\partial_{\mu}-i g A_{\mu}\right)(U \Psi)-m_{4} \bar{\Psi} \Psi-\left(m_{1}-m_{4}\right) \bar{\Psi}_{5} \Psi_{5},
$$

where we introduced the 5-plet

$$
\Psi=\left(\begin{array}{l}
\psi_{4} \\
\psi_{1}
\end{array}\right)
$$

and we denoted by $\Psi_{5}$ the fifth component of $\Psi$, namely $\Psi_{5}=\psi_{1}$, while $A_{\mu}$ represents the elementary gauge fields in a compact notation. A simple field redefinition, $\Psi \rightarrow \Psi^{\prime} \equiv U^{\dagger} \Psi$, shows that the only dependence on the Goldstone fields in the composite fermion Lagrangian is associated to the mass term

$$
\mathcal{L}_{\text {comp }}^{c=1 / \sqrt{2}} \supset-\left(m_{1}-m_{4}\right)\left(\bar{\Psi}^{\prime} U\right)_{5}\left(U^{\dagger} \Psi^{\prime}\right)_{5},
$$

which gives the mass splitting between the 4-plet and the singlet. Notice that this property is a consequence of our choice of $c$, in the general Lagrangian the dependence on the Goldstones in the kinetic terms of the composite fields can not be removed. It is clear that, if $m_{1}=m_{4}$, in the composite sector Lagrangian an additional $\mathrm{SO}(5)$ symmetry is present, which allows us to remove the Higgs VEV.

With the same redefinition of the composite fields the Lagrangian for the elementary states in eq. (2.9) becomes

$$
\begin{aligned}
\mathcal{L}_{\text {elem }}^{c=1 / \sqrt{2}}= & i \bar{q}_{L} \not D q_{L}+i \bar{t}_{R} \not D t_{R} \\
& +y_{L 4} f \bar{q}_{L}^{5} \Psi^{\prime}+\left(y_{L 1}-y_{L 4}\right) f\left(\bar{q}_{L}^{5} U\right)_{5}\left(U^{\dagger} \Psi^{\prime}\right)_{5} \\
& +y_{R 4} f \bar{t}_{R}^{5} \Psi^{\prime}+\left(y_{R 1}-y_{R 4}\right) f\left(\bar{t}_{R}^{5} U\right)_{5}\left(U^{\dagger} \Psi^{\prime}\right)_{5}+\text { h.c. }
\end{aligned}
$$

The Goldstones in this case appear only in association with the $\left(y_{L 1}-y_{L 4}\right) f$ and $\left(y_{R 1}-y_{R 4}\right) f$ mass mixings.

From the structure of the Lagrangian in eqs. (3.22) and (3.25) we can simply understand why no divergence arises in the fermion contribution to $\widehat{S}$. In order to generate an 
effect that feels EWSB the corresponding operator must necessarily include some insertions of the Lagrangian terms containing the Goldstones. For our choice of $c$ the Goldstones are always associated to mass operators and any insertion leads to a reduction of the degree of divergence. The $\widehat{S}$ parameter is naively logarithmically divergent at one loop, thus the extra mass insertions make it finite.

A similar protection mechanism is also present for the fermion corrections to the $Z \bar{b}_{L} b_{L}$ vertex. In the case in which $y_{L 1}=y_{L 4}$ the remaining $y_{L 4} f \bar{q}_{L}^{5} \Psi^{\prime}$ mixing is independent of the Goldstones. The only operators containing the $U$ matrix are the $\left(m_{1}-m_{4}\right)$ mass term and the $\left(y_{R 1}-y_{R 4}\right) f$ mixing. In order to generate a correction to $g_{b_{L}}$ some insertions of these operators are needed in addition to the four insertions of $y_{L 4}$. These extra mass insertions make the corrections to the $Z \bar{b}_{L} b_{L}$ vertex finite.

A similar structure of the effective Lagrangian is also present if $c=-1 / \sqrt{2}$. This case can be connected to the one we discussed with the redefinitions $\psi_{1} \rightarrow-\psi_{1}, y_{L, R 1} \rightarrow-y_{L, R 1}$, which just reverse the sign of $c$.

A particular implementation of our effective Lagrangian with $c=1 / \sqrt{2}$ has been studied in ref. [27]. In this work the additional relations $y_{L 4}=y_{L 1}$ and $y_{R 4}=y_{R 1}$ are assumed. In this particular case the only dependence on the Goldstones comes from the mass splitting term between the composite 4-plet and the singlet. The explicit computation of the fermion corrections to the $Z \bar{b}_{L} b_{L}$ vertex presented in ref. [27] shows that the new physics contributions are finite, in agreement with the results of our analysis.

\section{Results in explicit models}

After the general analysis presented in the previous section, we now focus on a more detailed study of the corrections to the EW precision parameters in some explicit scenarios. First of all we will consider the simplified set-ups in which only one light composite multiplet is present in the effective theory. Afterwards we will study two more complete models containing a composite 4-plet as well as a singlet.

The analysis of explicit scenarios is of course essential to obtain a reliable quantitative determination of the constraints coming from the EW precision data. Moreover it allows to check the validity of the general results derived in the previous section.

In all our numerical results we fix the top mass to the value $m_{t}=m_{t}^{\overline{M S}}(2 \mathrm{TeV})=$ $150 \mathrm{GeV}$, which corresponds to the pole mass $m_{t}^{\text {pole }}=173 \mathrm{GeV}$. Moreover, to estimate the constraints from the oblique parameters, we chose a cut-off scale $m_{*}=3 \mathrm{TeV}$.

\subsection{The case of a light singlet}

As a first example we consider the case in which only a light composite singlet is present in the effective theory. The effective Lagrangian for this set-up can be easily read from the general one of section 2 by removing the terms containing $\psi_{4}$. In this configuration the resonance spectrum contains only one composite state, the $\widetilde{T}$, which has the same electric charge as the top and a mass

$$
m_{\widetilde{T}}^{2}=m_{1}^{2}+y_{R 1}^{2} f^{2}
$$


We start our analysis by considering the corrections to the $\widehat{S}$ parameter. In the general analysis we saw that the fermion contributions to $\widehat{S}$ can diverge only if the spectrum contains a light 4-plet, thus in our present set-up we expect a finite result. In fact at leading order in the $v / f$ expansion we find that the one-loop fermion contribution is given by

$$
\Delta \widehat{S}_{\text {ferm }}=\frac{g^{2}}{192 \pi^{2}} \xi \frac{m_{1}^{2} y_{L 1}^{2} f^{2}}{\left(m_{1}^{2}+y_{R 1}^{2} f^{2}\right)^{2}}\left[-5+2 \log \left(\frac{2\left(m_{1}^{2}+y_{R 1}^{2} f^{2}\right)^{2}}{v^{2} y_{L 1}^{2} y_{R 1}^{2} f^{2}}\right)\right] .
$$

Notice that the argument of the logarithm can be identified with the ratio between the mass of the heavy fermion resonance $m_{\widetilde{T}}$ and the top mass.

$$
m_{t}^{2} \simeq \frac{v^{2} y_{L 1}^{2} y_{R 1}^{2} f^{2}}{2\left(m_{1}^{2}+y_{R 1}^{2} f^{2}\right)} .
$$

For typical values of the parameters, $y_{L 1} \sim y_{R 1} \sim 1, m_{1} \lesssim 1 \mathrm{TeV}$ and $\xi \lesssim 0.2$, the contribution in eq. (4.2) is positive and small, $\Delta \widehat{S}_{\text {ferm }} \lesssim 10^{-4}$.

As we discussed in section 3 , although the correction to $\widehat{S}$ coming from the low-energy dynamics is calculable, large uncalculable UV contributions can be present. Even if we assume that the tree-level effects given in eq. (3.1) are negligible, the loop contributions coming from the UV dynamics (see the estimate in eq. (3.5)) are typically dominant with respect to the corrections in eq. (4.2). We can check that the UV effects can be important by slightly modifying our explicit computation. We consider an effective theory in which a composite 4-plet is present as well as a singlet. In order to recover the case with only a light singlet, we then take the limit in which the 4-plet mass is sent to the cut-off $m_{*}$. To ensure that $\widehat{S}$ is calculable in the effective theory we set $c^{2}=1 / 2$. The explicit computation of $\Delta \widehat{S}$ leads to the result in eq. (4.2) plus an additional shift which, at the leading order in an expansion in the cut-off, is given by

$$
\Delta \widehat{S}_{\mathrm{ferm}}^{U V}=-\frac{g^{2}}{24 \pi^{2}} \xi \simeq-1.8 \cdot 10^{-3} \xi .
$$

As expected, the 4-plet does not decouple in the limit in which it becomes heavy. The UV corrections in eq. (4.4) have a size compatible with our estimate in eq. (3.5) and are typically larger than the singlet contribution in eq. (4.2). Notice that the result in eq. (4.4) gives only an example of possible UV effects and should not be thought as a complete determination of the UV contributions. In order to properly compute the total shift in $\widehat{S}$ the whole UV completion of the model should be taken into account.

Let us now consider the $\widehat{T}$ parameter. As shown in the general analysis, the fermion corrections are finite and saturated by the low-energy contributions. The explicit calculation gives the following result at leading order in $v / f$ :

$$
\Delta \widehat{T}_{\text {ferm }}=\frac{3 \xi}{64 \pi^{2}} \frac{y_{L 1}^{4} m_{1}^{2} f^{2}}{\left(m_{1}^{2}+y_{R 1}^{2} f^{2}\right)^{3}}\left\{m_{1}^{2}+2 y_{R 1}^{2} f^{2}\left[\log \left(\frac{2\left(m_{1}^{2}+y_{R 1}^{2} f^{2}\right)^{2}}{v^{2} y_{L 1}^{2} y_{R 1}^{2} f^{2}}\right)-1\right]\right\} .
$$

This contribution is positive and, in a large part of the parameter space, can compensate the negative shift that comes from the non-linear Higgs dynamics (see eq. (3.7)). In the points in which $y_{L 1} \sim y_{R 1} \sim 1$, the estimate given in eq. (3.8) is approximately valid. 


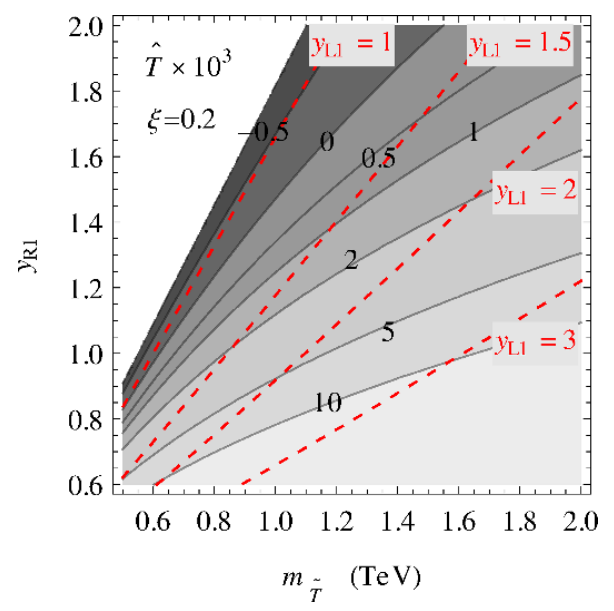

Figure 8. Corrections to the $\widehat{T}$ parameter as a function of the singlet mass $m_{\widetilde{T}}$ and of the $y_{R 1}$ mixing. The result corresponds to the case with only a light singlet and includes the contribution due to the Higgs non-linear dynamics in eq. (3.7) and the exact fermion one-loop correction. The compositeness scale has been fixed to the value $\xi=0.2$. The red dashed lines correspond to the contours with fixed $y_{L 1}$.

The total shift in $\widehat{T}$ is shown in figure 8 for the reference value $\xi=0.2$, corresponding to $f=550 \mathrm{GeV}$. It can be seen that sizable positive values of $\Delta \widehat{T}$ can easily be obtained for reasonable values of the singlet mass and of the elementary-composite mixings.

Finally we analyze the corrections to the $Z \bar{b}_{L} b_{L}$ vertex. We showed in section 3 that in the case with only a light singlet the one-loop fermion corrections to this observable are finite. The absence of a 4-plet also implies that additional contributions coming from 4 -fermion operators and from the UV dynamics are suppressed by the cut-off scale and can be expected to be negligible. At leading order in $v / f$ we find that the shift in $g_{b_{L}}$ is given by

$$
\delta g_{b_{L}}=\frac{\xi}{64 \pi^{2}} \frac{y_{L 1}^{4} m_{1}^{2} f^{2}}{\left(m_{1}^{2}+y_{R 1}^{2} f^{2}\right)^{3}}\left\{m_{1}^{2}+2 y_{R 1}^{2} f^{2}\left[\log \left(\frac{2\left(m_{1}^{2}+y_{R 1}^{2} f^{2}\right)^{2}}{v^{2} y_{L 1}^{2} y_{R 1}^{2} f^{2}}\right)-1\right]\right\} .
$$

Comparing this result with the fermion contribution to $\widehat{T}$ in eq. (4.5) we can notice that a strict relation exists between the two quantities $\Delta \widehat{T}_{\text {ferm }}=3 \delta g_{b_{L}} \cdot{ }^{12}$ In particular the positive correction to $\widehat{T}$ is related to a corresponding positive shift in $g_{b_{L}}$. For the typical size of the fermion contribution to $\widehat{T}$ needed to satisfy the experimental bounds, $1 \cdot 10^{-3}<\Delta \widehat{T}<$ $2 \cdot 10^{-3}$, a moderate contribution to $\delta g_{b_{L}}$ is found: $g_{b_{L}}: 0.33 \cdot 10^{-3}<\delta g_{b_{L}}<0.66 \cdot 10^{-3}$. As we already discussed (see figure 6), the experimental measurements disfavor a positive contribution to the $Z \bar{b}_{L} b_{L}$ coupling. Thus the scenario with only a light singlet tends to be in worse agreement with the EW precision data than the SM.

On the other hand, if we neglect the constraints on $\delta g_{b_{L}}$ and only consider the bounds on the oblique EW parameters, it is not hard to satisfy the experimental constraints even for sizable values of $\xi$.

\footnotetext{
${ }^{12}$ This relation was already noticed in refs. [47, 54].
} 


\subsection{The case of a light 4-plet}

As a second simplified scenario we consider the case in which the resonance spectrum contains only a light 4-plet. The general analysis of section 3 showed that in this case only $\widehat{T}$ receives a finite contribution from fermion loops, whereas the corrections to the $\widehat{S}$ parameter and to the $Z \bar{b}_{L} b_{L}$ vertex are logarithmically divergent. ${ }^{13}$

Before discussing in details the contributions to the EW parameter, we analyze the spectrum of the resonances. The 4-plet gives rise to two $\mathrm{SU}(2)_{L}$ doublets with hypercharges $1 / 6$ and $7 / 6$. The $\mathbf{2}_{1 / 6}$ doublet contains a top partner $T$ and a bottom partner $B$, while the $\mathbf{2}_{7 / 6}$ doublet contains an exotic state with charge $5 / 3\left(X_{5 / 3}\right)$ and a top resonance $\left(X_{2 / 3}\right)$. The mixing with the elementary states induces a mass splitting between the two doublets. The states inside each doublet, instead, receive only a small splitting due to EWSB effects and are nearly degenerate in mass. In particular the $B$ and $X_{5 / 3}$ states are not coupled to the Higgs and their masses do not receive corrections after EWSB. The masses of the composite resonances are given by

$$
m_{X_{2 / 3}}^{2} \simeq m_{X_{5 / 3}}^{2}=m_{4}^{2} \quad \text { and } \quad m_{T}^{2} \simeq m_{B}^{2}=m_{4}^{2}+y_{L 4}^{2} f^{2} .
$$

The top mass at the leading order in $v / f$ is given by

$$
m_{t}^{2} \simeq \frac{v^{2} y_{L 4}^{2} y_{R 4}^{2} f^{2}}{2\left(m_{4}^{2}+y_{L 4}^{2} f^{2}\right)} .
$$

The dominant contribution to the $\widehat{S}$ parameter comes from the logarithmically enhanced corrections due to loops of fermion resonances. The explicit result can be obtained from eq. (3.4) by setting $c=0:{ }^{14}$

$$
\Delta \widehat{S}_{\mathrm{ferm}}=\frac{g^{2}}{8 \pi^{2}} \xi \log \left(\frac{m_{*}^{2}}{m_{4}^{2}}\right) \simeq 1.6 \cdot 10^{-2} \xi,
$$

where the numerical estimate has been obtained by setting $m_{4} \simeq 700 \mathrm{GeV}$ and $m_{*} \simeq 3 \mathrm{TeV}$. If the gauge resonances are heavy, $m_{*} / f=g_{*} \gtrsim 4$, the correction in eq. (4.9) is comparable or even larger than the tree-level one in eq. (3.1).

The sizable positive contribution to the $\widehat{S}$ parameter implies a quite stringent bound on the compositeness scale, $\xi \lesssim 0.1$ (see figure 2 ). An even stronger constraint is obtained if we also consider the corrections to the $\widehat{T}$ parameter. The full expression of the fermion contributions at leading order in $v / f$ is in this case too involved and does not give useful insights, so we only report here the leading term in the $y$ expansion:

$$
\Delta \widehat{T}_{\text {ferm }}=-\frac{\xi}{32 \pi^{2}} \frac{y_{L 4}^{4} f^{2}}{m_{4}^{2}} .
$$

\footnotetext{
${ }^{13}$ The corrections to the $\widehat{T}$ parameter and to the $Z \bar{b}_{L} b_{L}$ vertex in this set-up have been studied also in ref. [54]. The results for $\widehat{T}$ are similar to the ones we find. The results for the $Z \bar{b}_{L} b_{L}$ corrections are also in agreement with ours if we exclude the contributions from 4 -fermion operators which are not included in the analysis of ref. [54].

${ }^{14}$ The same result can be obtained with the following equivalent procedure. We consider an effective theory containing a 4 -plet and a singlet with $c^{2}=1 / 2$. In this case the fermion contribution to $\widehat{S}$ is finite and calculable. The explicit computation shows that a contribution of the form $g^{2} /\left(8 \pi^{2}\right) \xi \log \left(m_{1}^{2} / m_{4}^{2}\right)$ is present. In the limit in which the singlet becomes heavy, $m_{1} \rightarrow m_{*}$, we recover, as expected, the contribution in eq. (4.9).
} 


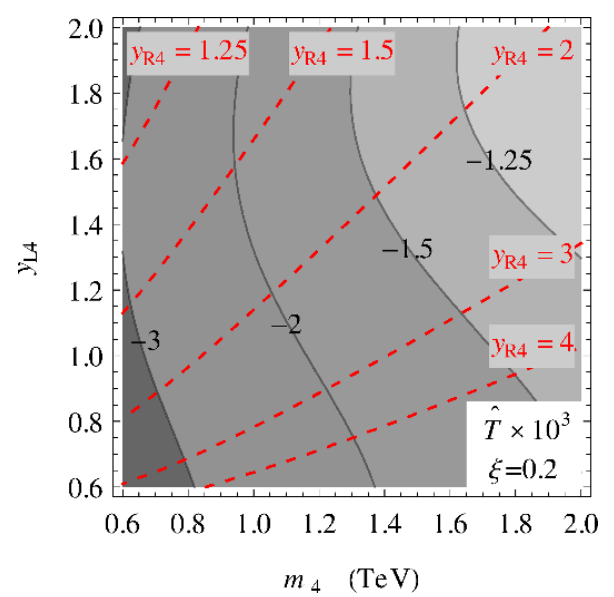

Figure 9. Corrections to the $\widehat{T}$ parameter as a function of the mass parameter $m_{4}$ and of the $y_{L 4}$ mixing. The result corresponds to the case with only a light 4-plet and includes the contribution due to the Higgs non-linear dynamics in eq. (3.7) and the exact fermion one-loop correction. The compositeness scale has been fixed to the value $\xi=0.2$. The red dashed lines correspond to the contours with fixed $y_{R 4}$.

The approximate result suggests that the shift in $\widehat{T}$ is negative. This conclusion is typically correct and has been explicitly verified with a numerical computation. The main contributions to $\widehat{T}$ coming from the non-linear Higgs dynamics (see eq. (3.7)) and from fermion loops are shown in figure 9 for $\xi=0.2$. Similar results are obtained for different values of $\xi$. Notice that the leading order expression in eq. (4.10) capture only the overall size of the fermion contributions. The exact result can deviate from the estimate at order one especially in the parameter space region in which $y_{R 4}$ becomes large.

The fact that the shift in $\widehat{T}$ is necessarily negative makes the constraints coming from the oblique parameters extremely severe. Using the results in figure 2 an upper bound $\xi \lesssim 0.02$ at the $99 \%$ confidence level is obtained, which corresponds to a lower bound $f \gtrsim 1.7 \mathrm{TeV}$.

Although the configuration with only a light 4-plet is strongly disfavored by the large corrections to the oblique parameters, it is still worth discussing the form of the corrections to the $Z \bar{b}_{L} b_{L}$ vertex. The explicit computation will be useful to verify the results obtained in our general analysis in section 3 .

We start by considering the contributions related to the leading-order terms in the effective Lagrangian. If we neglect the effects coming from higher-dimensional operators and from 4 -fermion contact interactions, we get the following corrections to the $Z \bar{b}_{L} b_{L}$ vertex at the leading order in the $v / f$ expansion:

$$
\begin{aligned}
\delta g_{b_{L}}^{4-\text { plet }}= & -\frac{\xi}{32 \pi^{2}} \frac{y_{L 4}^{2} y_{R 4}^{2} f^{2}}{m_{4}^{2}+y_{L 4}^{2} f^{2}}\left[\frac{y_{L 4}^{2} f^{2}}{m_{4}^{2}+y_{L 4}^{2} f^{2}}+\left(1-\frac{y_{R 4}^{2} f^{2}}{4 m_{4}^{2}}\right) \log \left(1+\frac{y_{L 4}^{2} f^{2}}{m_{4}^{2}}\right)\right. \\
& \left.-y_{L 4}^{2} f^{2} \frac{4 m_{4}^{2}\left(m_{4}^{2}+y_{L 4}^{2} f^{2}\right)-\left(2 m_{4}^{2}+y_{L 4}^{2} f^{2}\right) y_{R 4}^{2} f^{2}}{4 m_{4}^{2}\left(m_{4}^{2}+y_{L 4}^{2} f^{2}\right)^{2}} \log \left(\frac{2\left(m_{4}^{2}+y_{L 4}^{2} f^{2}\right)^{2}}{v^{2} y_{L 4}^{2} y_{R 4}^{2} f^{2}}\right)\right]
\end{aligned}
$$


As expected, due to the selection rule discussed in subsection 3.2, the fermion contribution to the $g_{b_{L}}$ coupling is finite.

If higher-order operators and in particular higher-order mixings between the elementary and the composite states are present in the effective Lagrangian, the selection rule can be violated and sizable corrections to the result in eq. (4.11) can arise. This is a signal of the fact that the $Z \bar{b}_{L} b_{L}$ vertex is sensitive to the UV dynamics of the theory. To explicitly verify this property we can use a procedure analogous to the one we adopted for the $\widehat{S}$ parameter in the case with only a light singlet. We consider a theory with a 4-plet as well as a singlet and then we recover the configuration with only a light 4-plet by taking the limit in which the singlet mass goes to the cut-off $m_{*}$. Using this procedure we find that the fermion correction to the $Z \bar{b}_{L} b_{L}$ vertex contains an additional contribution with respect to the result in eq. (4.11):

$$
\delta g_{b_{L}}=\delta g_{b_{L}}^{4-\text { plet }}+\frac{\xi}{32 \pi^{2}} \frac{y_{L 4}^{2} f^{2}}{m_{4}^{2}+y_{L 4}^{2} f^{2}} c^{2} y_{L 1}\left(y_{L 1}-\sqrt{2} c y_{L 4}\right) .
$$

The additional contribution arises at leading order in the $y$ expansion and is independent of the singlet mass, it only depends on the mixing of the singlet with the elementary states $y_{L 1}$.

An equivalent way to understand the non-decoupling of the singlet is the following. In the limit in which the singlet becomes heavy we can integrate it out from the effective theory. This procedure generates a set of higher-order operators, in particular it gives rise to a term of the form $\left(y_{L 1} c / m_{*}\right)\left(q_{L}^{\mathbf{5}} U\right)_{5} \gamma^{\mu} d_{\mu}^{i} \psi_{4}^{i}+$ h.c., where we replaced the singlet mass by the cut-off $m_{*}$. This higher-order mixing couples the $q_{L}$ doublet with the left-handed component of the composite 4-plet and induces a breaking of the $Z \bar{b}_{L} b_{L}$ selection rule, as can be easily inferred from the discussion in subsection 3.2 .

Notice that in the case in which $c=0$ the higher-dimension operators are not generated by integrating out the singlet, thus the selection rule is still unbroken and the additional correction to the $Z \bar{b}_{L} b_{L}$ vertex in eq. (4.12) vanishes. There is also a second case in which the additional corrections are not there. As we saw in subsection 3.3, if $c= \pm 1 / \sqrt{2}$ and $y_{L 1}= \pm y_{L 4}$ the low-energy theory acquires an extra symmetry which protects the EW observables. In this case we expect the decoupling of the heavy dynamics to occur and, in fact, the extra correction in eq. (4.12) exactly cancels.

To conclude the analysis of the case with only a light 4-plet we now consider the effects due to the 4-fermion contact operators. As expected, vertices of the form given in eq. (3.17) induce a finite correction to the $Z \bar{b}_{L} b_{L}$ vertex:

$$
\begin{aligned}
\delta g_{b_{L}}^{4-\text { ferm }}= & \frac{3 e_{L 4} \xi y_{L 4}^{2} f^{2}}{64 \pi^{2}\left(m_{4}^{2}+y_{L 4}^{2} f^{2}\right)^{3}}\left\{m_{4}^{2} y_{L 4}^{2}\left(m_{4}^{2}+y_{L 4}^{2} f^{2}-4 y_{R 4}^{2} f^{2}\right)\right. \\
& \left.\left.\left.+2 y_{R 4}^{2}\left[\left(m_{4}^{2}+y_{L 4}^{2} f^{2}\right)^{2} \log \left(\frac{m_{4}^{2}+y_{L 4}^{2} f^{2}}{m_{4}^{2}}\right)+y_{L 4}^{4} f^{4} \log \left(\frac{v^{2} y_{L 4}^{2} y_{R 4}^{2} f^{2}}{2\left(m_{4}^{2}+y_{L 4}^{2} f^{2}\right)^{2}}\right)\right] \text {. }\right] .\right\} .\right)
\end{aligned}
$$

On the other hand, the vertex in eq. (3.18) induces a logarithmically divergent contribution:

$$
\delta g_{b_{L}}^{4-\text { ferm }}=\frac{3 e_{R 4}}{32 \pi^{2}} \xi \frac{y_{L 4}^{2} f^{2}}{m_{4}^{2}+y_{L 4}^{2} f^{2}} y_{L 4}^{2} \log \left(\frac{m_{*}^{2}}{m_{4}^{2}}\right) .
$$


Notice that the results in eqs. (4.13) and (4.14) correspond to the case in which the 4fermion vertex has the structure $\left(\bar{B}_{L}^{a} \gamma^{\mu} B_{L}^{a}\right)\left(\bar{T}^{b} \gamma_{\mu} T^{b}+\bar{X}_{2 / 3}^{b} \gamma_{\mu} X_{2 / 3}^{b}\right)$, where $a$ and $b$ are color indices. Different color structures lead to results that only differ by group theory factors. ${ }^{15}$

The sign of the 4-fermion contribution crucially depends on the sign of the coefficients $e_{L, R}$. In our low-energy effective theory $e_{L, R}$ are completely free parameters, thus their sign is not fixed. From the UV perspective, instead, the operators in eqs. (3.17) and (3.18) arise from the exchange of heavy bosonic resonances and the sign of their coefficients is usually fixed by the quantum numbers of the resonances. It can be checked that the $e_{L, R}$ coefficients can be generated with arbitrary sign by considering resonances in different representations of $\mathrm{SO}(4)$.

\subsection{Two complete models}

In this subsection we finally consider two more complete models that include both a 4-plet and a singlet. In order to reduce the number of parameters we choose a common value for the left and right elementary mixings: $y_{L 4}=y_{L 1}=y_{L}$ and $y_{R 4}=y_{R 1}=y_{R}$. In this case the fermion Lagrangian (excluding the interactions with the gauge fields) becomes equal to the one of the 2 -site model proposed in refs. [13, 66].

An interesting byproduct of this choice is the fact that the fermion contribution, which dominates the Higgs potential, becomes only logarithmically divergent. One renormalization condition is enough to regulate the divergence and one can fix it by choosing the compositeness scale $f$. In this way the Higgs mass becomes calculable and an interesting relation between $m_{h}$ and the masses of the top partners holds [13]:

$$
\frac{m_{h}}{m_{t}} \simeq \frac{\sqrt{2 N_{c}}}{\pi} \frac{m_{T} m_{\widetilde{T}}}{f} \sqrt{\frac{\log \left(m_{T} / m_{\widetilde{T}}\right)}{m_{T}^{2}-m_{\widetilde{T}}^{2}}},
$$

where $m_{T}$ is the mass of the states in the $\mathbf{2}_{1 / 6}$ doublet coming from the 4-plet and $m_{\widetilde{T}}$ is the mass of the heavy singlet after the mixing with the elementary states. The complete spectrum of the composite resonances is a combination of the ones described in the cases with only one light multiplet considered in the previous subsections. The complete mass matrix for the charge $2 / 3$ states is given by

$$
M=\left(\begin{array}{cccc}
0 & -\frac{1}{2} y_{L 4} f\left(c_{h}+1\right) & \frac{1}{2} y_{L 4} f\left(c_{h}-1\right) & \frac{1}{\sqrt{2}} y_{L 1} f s_{h} \\
-\frac{1}{\sqrt{2}} y_{R 4} f s_{h} & m_{4} & 0 & 0 \\
\frac{1}{\sqrt{2}} y_{R 4} f s_{h} & 0 & m_{4} & 0 \\
-y_{R 1} f c_{h} & 0 & 0 & m_{1}
\end{array}\right)
$$

where $c_{h} \equiv \cos (\langle h\rangle / f)$ and $s_{h} \equiv \sin (\langle h\rangle / f)$. The relation in eq. (4.15) allows us to fix the mass of one heavy multiplet as a function of the other parameters of the effective Lagrangian. Another mass parameter can be fixed by the requirement of reproducing the

\footnotetext{
${ }^{15}$ The combination of $T$ and $X_{2 / 3}$ is dictated by the $P_{L R}$ symmetry which is unbroken in the composite sector.
} 
top mass. At the leading order in the $v / f$ expansion we find that $m_{t}$ is given by

$$
m_{t}^{2}=\frac{v^{2}\left(m_{4}-m_{1}\right)^{2} y_{L}^{2} y_{R}^{2} f^{2}}{2\left(m_{4}^{2}+y_{L}^{2} f^{2}\right)\left(m_{1}^{2}+y_{R}^{2} f^{2}\right)} .
$$

Apart from the masses of the composite multiplets and the elementary mixings, only one free parameter appears in the effective Lagrangian: the coefficient of the $d$-symbol term, $c$. In the following we will analyze the models obtained for two particular choices of $c$. The first one is the case $c=0$ which exactly corresponds to the 2 -site model of refs. $[13,66]$. The second case corresponds to the choice $c=1 / \sqrt{2}$ which, as explained in subsection 3.3, implies the presence of an additional protection for the EW parameters. This second choice reproduces the model studied in ref. [27].

The case $\boldsymbol{c}=\mathbf{0}$. We start by considering the 2 -site model $(c=0)$. In this case the leading corrections to the $\widehat{S}$ parameter are the same as in the case with only one light 4-plet. As shown in section 3, the constraints on $\widehat{S}$ alone are strong enough to put an absolute upper bound on the compositeness scale $\xi \lesssim 0.1$, as can be seen from figure 3 .

Let us now consider the $\widehat{T}$ parameter. We can reduce the number of free parameters by fixing the top and Higgs masses. The requirement of reproducing the correct Higgs mass gives a relation between $m_{T}$ and $m_{\widetilde{T}}$ (see eq. (4.15)), while fixing the top mass allows us to determine the right mixing $y_{R}$ as a function of the other parameters. With this procedure we are left with only two free parameters, which we choose to be $m_{T}$ and the $q_{L}$ compositeness angle $\phi_{L}$ defined as

$$
\sin \phi_{L} \equiv \frac{y_{L} f}{\sqrt{m_{4}^{2}+y_{L}^{2} f^{2}}} .
$$

Notice that with this procedure the right mixing $y_{R}$ is determined up to a twofold ambiguity. In the figures that show the numerical results we will thus include two plots that correspond to the two choices of $y_{R}$.

The corrections to the $\widehat{T}$ parameter are shown in figure 10 for $\xi=0.1$. To obtain the numerical results we fixed the Higgs mass to the value $m_{h}=126 \mathrm{GeV} .{ }^{16}$ As expected from the results we discussed in the previous simplified cases, in the region in which the 4-plet is the lightest multiplet the corrections to $\widehat{T}$ are negative, whereas a light singlet typically implies a positive shift. The fit of the oblique parameters can put strong bounds on the parameter space of the model. In the plots we showed the allowed regions for $68 \%$ and $95 \%$ confidence level. To obtain the constraints we estimated $\widehat{S}$ by adding the leading corrections in eqs. (3.1), (3.2) and (3.4) for the choice $m_{*}=3 \mathrm{TeV}$.

The numerical results show that the oblique parameters can be used to set some lower bounds on the masses of the resonances coming from the composite 4-plet. At the $95 \%$ confidence level one finds $m_{X_{2 / 3}} \simeq m_{X_{5 / 3}} \gtrsim 0.95 \mathrm{TeV}$ for the masses of the exotic doublet $\mathbf{2}_{7 / 6}$ and $m_{T} \simeq m_{B} \gtrsim 1.2 \mathrm{TeV}$ for the $\mathbf{2}_{1 / 6}$ states. If we assume a $25 \%$ cancellation in the corrections to the $\widehat{S}$ parameter the bounds are significantly relaxed:

\footnotetext{
${ }^{16}$ For simplicity we do not take into account the running of the Higgs mass.
} 

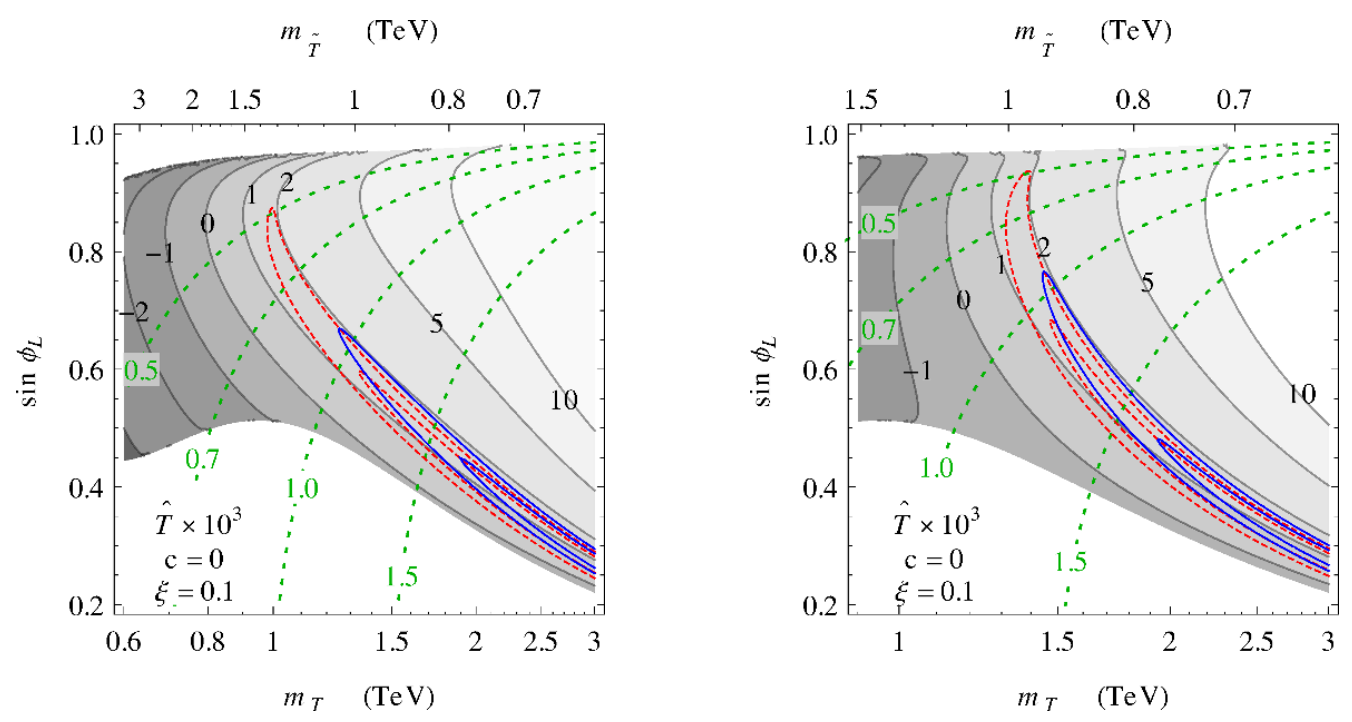

Figure 10. Corrections to the $\widehat{T}$ parameter as a function of the mass of the top partners and of the $q_{L}$ compositeness in the model with $c=0$ for $\xi=0.1$. The two plots correspond to the two different choices of $y_{R}$ that allow to obtain the correct Higgs and top masses at fixed $m_{T}$ and $\phi_{L}$ (see the main text for further details). In the white regions at the top and at the bottom of the plots the Higgs and top masses can not be reproduced. The dashed green contours show the mass (in $\mathrm{TeV}$ ) of the exotic composite state $X_{5 / 3}$. The solid blue contours give the regions that pass the constraints on the oblique parameters at the $68 \%$ and $95 \%$ confidence level, while the dashed red lines show how the bounds are modified if we assume a $25 \%$ reduction of $\widehat{S}$.

$m_{X_{2 / 3}} \simeq m_{X_{5 / 3}} \gtrsim 0.5 \mathrm{TeV}$ and $m_{T} \simeq m_{B} \gtrsim 1 \mathrm{TeV}$. Notice that these bounds are competitive or even stronger than the ones obtained from direct searches. For instance the current bounds on the exotic top partners is $m_{X_{5 / 3}} \gtrsim 700 \mathrm{GeV}$ [43-46].

Let us finally discuss the corrections to the $Z \bar{b}_{L} b_{L}$ vertex. The presence of a 4 -plet in the low-energy spectrum makes this observable sensitive to the UV dynamics of the theory and to possible 4-fermion interactions present in the effective Lagrangian. In particular, as discussed in the general analysis of section 3, logarithmically divergent contributions can arise from a set of 4 -fermion interactions.

If we neglect the UV contributions and set to zero the 4-fermion operators we find that the shift in the $Z \bar{b}_{L} b_{L}$ vertex is positive and somewhat correlated with the corrections to $\widehat{T}$. As an example we show in the left panel of figure 11 the shift in $g_{b_{L}}$ for the configurations corresponding to the left plot in figure 10. One can see that the corrections become typically large and positive in the presence of a light singlet. The points that pass the constraints on the oblique parameters have a small positive shift in the $Z \bar{b}_{L} b_{L}$ vertex: $0.2 \cdot 10^{-3} \lesssim \delta g_{b_{L}} \lesssim 0.8 \cdot 10^{-3}$.

The UV contributions and the effects of 4 -fermion operators can however drastically change the above result. In the right panel of figure 11 we show how the previous result changes if we add to the low-energy Lagrangian the interaction

$$
\frac{e_{R 4}}{f^{2}}\left(\bar{B}_{L}^{a} \gamma^{\mu} B_{L}^{a}\right)\left(\bar{T}_{R}^{b} \gamma_{\mu} T_{R}^{b}+\bar{X}_{2 / 3 R}^{b} \gamma_{\mu} X_{2 / 3 R}^{b}\right)
$$



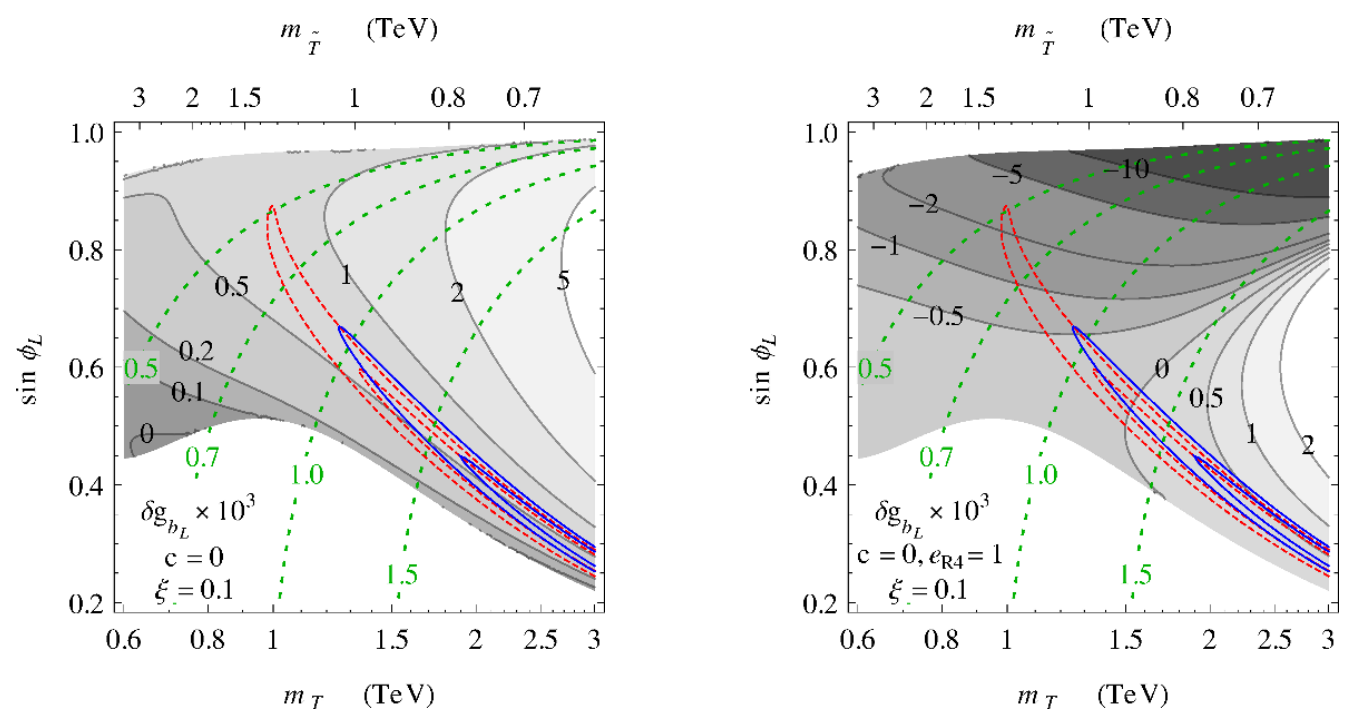

Figure 11. Corrections to the $Z \bar{b}_{L} b_{L}$ vertex in the model with $c=0$ for $\xi=0.1$. The results on the left panel are obtained by neglecting the UV effects and the contributions from 4-fermion operators. On the right panel we added the logarithmically enhanced contribution induced by the operator in eq. (4.19) with $e_{R 4}=1$. The configurations correspond to the ones chosen for the left plot in figure 10 .

with $e_{R 4}=1$. To obtain the numerical result we only included the leading logarithmically enhanced contribution to $\delta g_{b_{L}}$ and we set the cut-off to the value $m_{*}=3 \mathrm{TeV}$. As expected, the new correction strongly changes the result in the configurations with large $q_{L}$ compositeness, whereas the points with small $\phi_{L}$ are only marginally affected.

The case $c=1 / \sqrt{2}$. The second complete model we consider corresponds to the case $c=1 / \sqrt{2}$. In this set-up the EW observables are finite. In particular the main corrections to the $\widehat{S}$ parameter are given by the tree-level UV contributions and by the logarithmically enhanced corrections due to the non-linear Higgs dynamics. These corrections, for a reasonably high cut-off $\left(m_{*} \gtrsim 3 \mathrm{TeV}\right)$ are well below the absolute upper bound on $\widehat{S}$.

The corrections to the $\widehat{T}$ parameter are shown in figure 12. The configurations chosen for the plots correspond to the ones we used for the analogous plots in the case $c=0$ (see figure 10). The results, however, significantly differ in the two cases. In the case $c=1 / \sqrt{2}$ the corrections to $\widehat{T}$ tend to be more negative and a much lighter singlet is needed in order to pass the constraints on the oblique parameters $\left(m_{\widetilde{T}} \lesssim 0.8 \mathrm{TeV}\right)$. Notice that in this case the constraints are not significantly modified if we assume that some amount of cancellation in $\widehat{S}$ is present. Differently from the case $c=0$, the corrections to $\widehat{S}$ are small and are typically much below the absolute upper bound $\widehat{S} \lesssim 2.5 \cdot 10^{-3}$.

As in the case $c=0$, if we neglect the contributions from the UV dynamics and from the 4 -fermion operators, the corrections to the $Z \bar{b}_{L} b_{L}$ parameter tend to be positive and correlated to the shift in $\widehat{T}$. The numerical results in the plane corresponding to the right plot in figure 12 are shown in the left panel of figure 13. Due to the protection of the EW observables, the presence of 4-fermion operators can not induce logarithmically divergent 

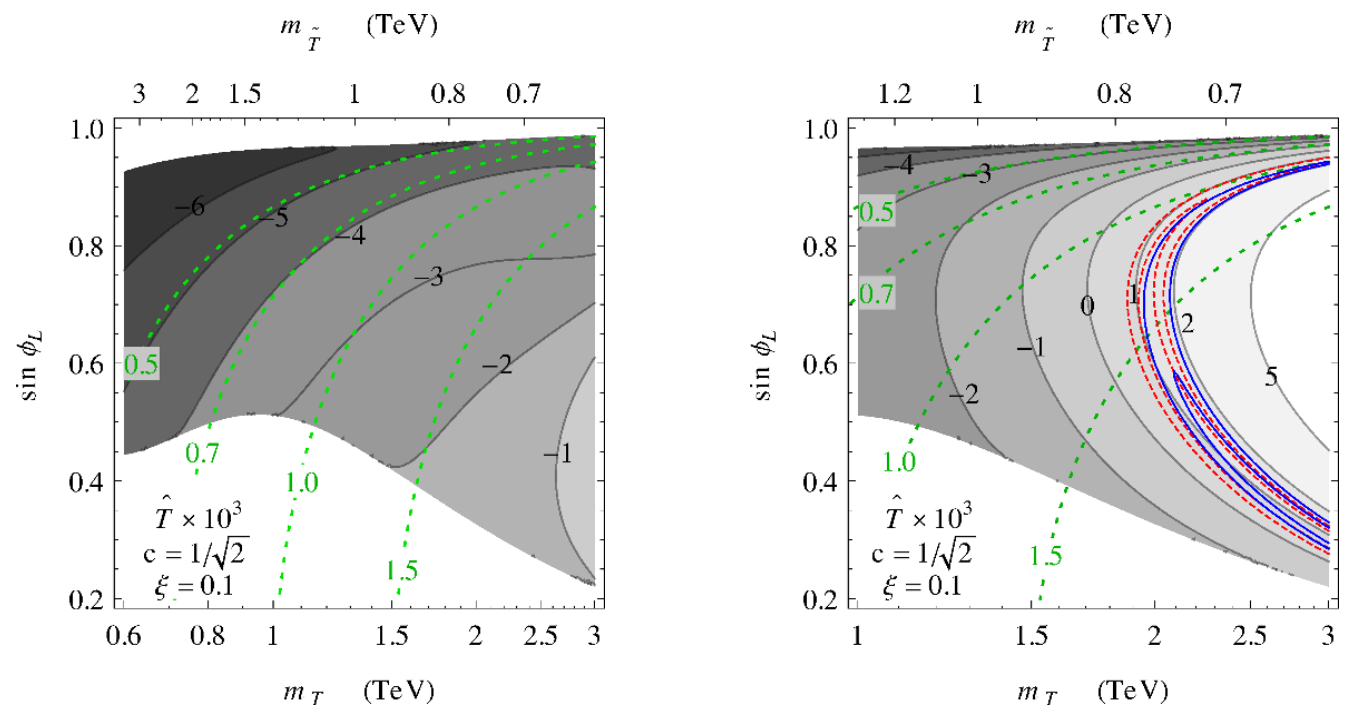

Figure 12. Corrections to the $\widehat{T}$ parameter as a function of the mass of the top partners and of the $q_{L}$ compositeness in the model with $c=1 / \sqrt{2}$ for $\xi=0.1$.
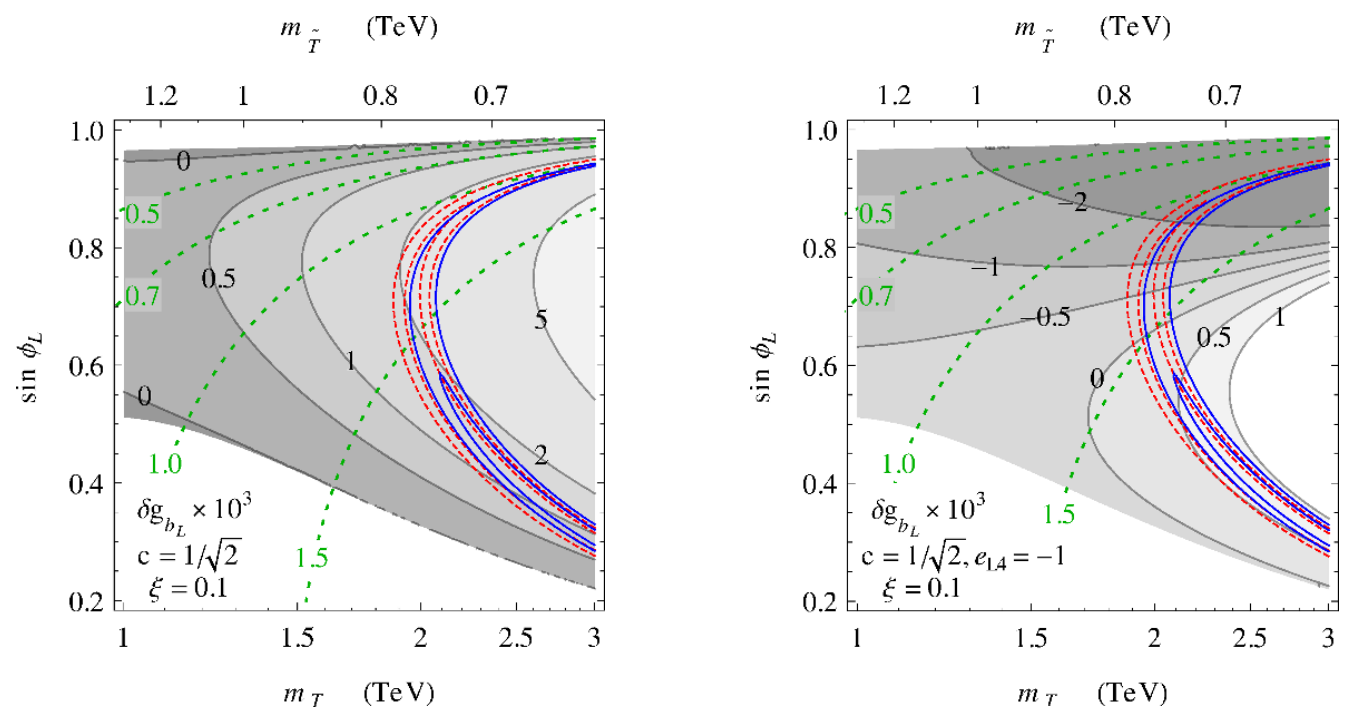

Figure 13. Corrections to the $Z \bar{b}_{L} b_{L}$ vertex in the model with $c=1 / \sqrt{2}$ for $\xi=0.1$. In the left plot we neglected the UV effects and the contributions from 4-fermion operators. On the right panel we added the shift induced by the operator in eq. (4.20) with $e_{L 4}=-1$. The configurations correspond to the one chosen for the right plot in figure 12.

contributions to the $Z \bar{b}_{L} b_{L}$ vertex. However sizable finite corrections are still possible. In the right panel of figure 13 we show how $\delta g_{b_{L}}$ is modified if we add the contributions due to the vertex

$$
\frac{e_{L 4}}{f^{2}}\left(\bar{B}_{L}^{a} \gamma^{\mu} B_{L}^{a}\right)\left(\bar{T}_{L}^{b} \gamma_{\mu} T_{L}^{b}+\bar{X}_{2 / 3 L}^{b} \gamma_{\mu} X_{2 / 3 L}^{b}\right),
$$

with $e_{L 4}=-1$. As expected, the corrections are large only in the parameter space region in which the $q_{L}$ has a large degree of compositeness. In this region the additional correction 
can easily induce a negative value for $\delta g_{b_{L}}$. Notice however that the sign of the corrections crucially depends on the sign of the coefficient of the 4 -fermion operators. In our effective approach this coefficient is a free parameter, but in a theory including a UV completion of our Lagrangian some constraints on the size and on the sign of the 4-fermion operators could be present.

\section{The case of a totally composite $t_{R}$}

So far we analyzed a class of models based on the standard implementation of partial compositeness in which all the SM fermions have a corresponding elementary counterpart. Of course, due to the quantum numbers of the left-handed SM fermions, including them in the effective Lagrangian via some elementary fields is the only reasonable option if we want to preserve the global $\mathrm{SO}(5)$ invariance in the composite sector. The situation is different for the right-handed fermions. They are singlets under the $\mathrm{SO}(4)$ symmetry and can be embedded in the theory as elementary fields or, alternatively, as chiral fermions coming from the strong dynamics. In this case the right-handed fermions are part of the composite sector and are total singlets under the global $\mathrm{SO}(5)$ invariance.

This alternative implementation of partial compositeness is particularly appealing for the right-handed top component. As shown in ref. [17] models with a totally composite $t_{R}$ can lead to minimally tuned implementations of the composite Higgs idea and can give rise to an interesting collider phenomenology [40].

In this section we analyze the corrections to the EW observables that are present in this alternative scenario. Our strategy will be similar to the one followed in the previous sections. We will use an effective Lagrangian approach to parametrize the low-energy dynamics of the models and we will analyze the EW parameters with particular attention to the corrections coming from the light composite fermions.

\subsection{The effective Lagrangian}

As we did for the models in section 2, we will concentrate on a minimal scenario in which the elementary top component is mixed with a composite operator that transforms in the fundamental representation of the global $\mathrm{SO}(5)$ symmetry. For simplicity we only include one level of composite resonances which transform as a 4-plet $\left(\psi_{4}\right)$ and a singlet $\left(\psi_{1}\right)$ under the $\mathrm{SO}(4)$ subgroup. The elementary sector of the theory contains the left-handed doublet $q_{L}$, while the $t_{R}$ is now an $\mathrm{SO}(5)$ chiral singlet belonging to the composite sector.

The effective Lagrangian for the composite states is given by ${ }^{17}$

$$
\begin{aligned}
\mathcal{L}_{\text {comp }}= & i \bar{\psi}_{4} \not D \psi_{4}+i \bar{\psi}_{1} \not D \psi_{1}+i \bar{t}_{R} \not D t_{R}-m_{4} \bar{\psi}_{4} \psi_{4}-m_{1} \bar{\psi}_{1} \psi_{1} \\
& +\left(i c_{L} \bar{\psi}_{4 L}^{i} \gamma^{\mu} d_{\mu}^{i} \psi_{1 L}+i c_{R} \bar{\psi}_{4 R}^{i} \gamma^{\mu} d_{\mu}^{i} \psi_{1 R}+\text { h.c. }\right)+\left(i c_{t} \bar{\psi}_{4 R}^{i} \gamma^{\mu} d_{\mu}^{i} t_{R}+\text { h.c. }\right)+\frac{1}{f^{2}}(\bar{\psi} \psi)^{2} .
\end{aligned}
$$

As in eq. (2.7), the covariant derivative for the 4-plet $\psi_{4}$ contains the CCWZ $e_{\mu}$ symbol: $D_{\mu} \psi_{4}=\left(\partial_{\mu}-2 / 3 i g^{\prime} X_{\mu}+i e_{\mu}\right) \psi_{4}$. Notice that a mass term of the form $m_{R} \bar{t}_{R} \psi_{1 L}+$ h.c. can

\footnotetext{
${ }^{17}$ The presence of chiral states coming from the strong dynamics does not allow us to impose a parity symmetry in the strong sector. For this reason in eq. (5.1) we wrote independent $d$-symbol interactions for the left- and right-handed chiralities.
} 
be added to the effective Lagrangian in eq. (5.1). This term can however be removed by a redefinition of the $\psi_{1 R}$ and $t_{R}$ fields. The Lagrangian containing the kinetic terms for the elementary fields and the mixings is

$$
\mathcal{L}_{\text {elem+mixing }}=i \bar{q}_{L} \not q_{L}+\left(y_{L t} f\left(\bar{q}_{L}^{\mathbf{5}}\right)^{I} U_{I 5} t_{R}+y_{L 4} f\left(\bar{q}_{L}^{\mathbf{5}}\right)^{I} U_{I i} \psi_{4}^{i}+y_{L 1} f\left(\bar{q}_{L}^{\mathbf{5}}\right)^{I} U_{I 5} \psi_{1}+\text { h.c. }\right) \text {. }
$$

Differently from the case with an elementary right-handed top, in the present scenario a direct mass mixing between the $q_{L}$ doublet and the $t_{R}$ singlet appears in the effective Lagrangian. The parameters in our effective Lagrangian are in general complex and some of the complex phases can not be removed by field redefinitions. For simplicity we assume that our theory is invariant under $C P$, in this way all the parameters in eqs. (5.1) and (5.2) are real.

An interesting question is whether the scenarios with totally composite $t_{R}$ can correspond to a particular limit of the case with an elementary $t_{R}$. To address this question we can notice that a property of the scenario with a totally composite right-handed top is the fact that the couplings and mixing of the $t_{R}$ field with the other composite resonances respect the $\mathrm{SO}(5)$ symmetry. The only breaking of the global invariance in the fermion sector comes from the mixings of the elementary doublet $q_{L}$ in eq. (5.2). In the case with an elementary $t_{R}$, instead, the $y_{R}$ mixings induce an extra source of $\mathrm{SO}(5)$ breaking. The different symmetry structure of the two implementations of partial compositeness clearly points out that the two scenarios are independent and can not be simply connected by a limiting procedure.

\section{$5.2 \quad$ Results}

We can now discuss the explicit results for the scenarios with a totally composite $t_{R}$. The analysis presented in section 3 can be straightforwardly adapted to the present set-up, in particular all the general results are still valid. Before presenting the numerical results for some simplified models, we briefly summarize the main differences with respect to the results of section 3 .

The contributions to the oblique parameters due to the non-linear Higgs dynamics (sse eqs. (3.2) and (3.7)) and the tree-level corrections to the $\widehat{S}$ parameter due to the gauge resonances (eq. (3.1)) are universal and do not depend on the assumptions on fermion compositeness. The presence of a light 4-plet of composite resonances still induces a logarithmically divergent contribution to the $\widehat{S}$ parameter, which is now given by

$$
\Delta \widehat{S}_{\mathrm{ferm}}^{\mathrm{div}}=\frac{g^{2}}{8 \pi^{2}}\left(1-c_{L}^{2}-c_{R}^{2}-c_{t}^{2}\right) \xi \log \left(\frac{m_{*}^{2}}{m_{4}^{2}}\right) .
$$

Notice that in this case the $d$-symbol involving the $t_{R}$ and the 4-plet can lead to a cancellation of the divergent contributions even if no light singlet is present in the spectrum. This cancellation happens for $c_{t}=1$.

As in the case with a partially composite $t_{R}$, the only couplings that break the custodial invariance and the $P_{L R}$ symmetry are the mixings of the elementary $q_{L}$. In the present case, however, we can write three mixings of this kind, $y_{L 4}, y_{L 1}$ and $y_{L t}$. The fermion 
contribution to the $\widehat{T}$ parameter is generated at order $y_{L}^{4}$, thus it is finite and dominated by the contributions coming from the lightest resonances.

The corrections to the $Z \bar{b}_{L} b_{L}$ vertex are in general logarithmically divergent. We can extend to the present set-up the discussion of subsection 3.2 and show that a selection rule exists also in this case. In particular a logarithmically divergent correction can be generated only by specific 4 -fermion operators and requires the presence of a light composite 4-plet. If the elementary $q_{L}$ is significantly composite non-decoupling effects can arise and the contribution from the UV dynamics can be sizable making the corrections to $g_{b_{L}}$ non predictable in the effective theory.

Notice that in the present set-up the top Yukawa is mainly determined by the $y_{L t}$ mixing. At the leading order in the $v / f$ expansion we find

$$
m_{t}^{2}=\frac{m_{4}^{2}}{m_{4}^{2}+y_{L 4}^{2} f^{2}} \frac{y_{L t}^{2} v^{2}}{2} .
$$

The presence of a direct mixing between the elementary doublet $q_{L}$ and the singlet $t_{R}$, allows to get the correct top mass even if we set to zero the $y_{L 4}$ and $y_{L 1}$ mixings. In this limit the composite 4-plet and singlet do not feel directly the breaking of the custodial and $P_{L R}$ symmetries and their corrections to the $\widehat{T}$ parameter and to the $Z \bar{b}_{L} b_{L}$ vertex are totally negligible. The contributions to $\widehat{S}$, instead, can still be sizable.

In the following we will consider in details two simplified scenarios, namely the cases in which only a light composite singlet or a light composite 4-plet are present in the effective theory.

The case of a light singlet. As a first simplified model we consider the case with only a light composite singlet. As we will see, in this limit the model with a totally composite $t_{R}$ has many properties in common with the case of a partially composite $t_{R}$ discussed in subsection 4.1.

The deviations in $\widehat{S}$ are dominated by the tree-level UV contribution and by the corrections due to the non-linear Higgs dynamics. For a high enough cut-off $\left(m_{*} \gtrsim 3 \mathrm{TeV}\right)$ the corrections to the $\widehat{S}$ parameter are well below the maximal value allowed by the EW precision tests.

The fermion contributions to the $\widehat{T}$ parameter can be sizable and are typically positive. At the leading order in $v / f$ they are given by

$$
\Delta \widehat{T}_{\text {ferm }}=\frac{3}{64 \pi^{2}} \xi \frac{y_{L 1}^{2} f^{2}}{m_{1}^{2}}\left\{y_{L 1}^{2}+2 y_{L t}^{2}\left[\log \left(\frac{2 m_{1}^{2}}{v^{2} y_{L t}^{2}}\right)-1\right]\right\}
$$

In figure 14 we show the total correction to $\widehat{T}$ including the leading IR effects given in eq. (3.7).

As in the analogous case with a partially composite $t_{R}$, the fermion contributions to the $Z \bar{b}_{L} b_{L}$ vertex are strongly correlated with the corrections to $\widehat{T}$. At leading order in $v / f$ we find

$$
\delta g_{b_{L}}=\frac{1}{64 \pi^{2}} \xi \frac{y_{L 1}^{2} f^{2}}{m_{1}^{2}}\left\{y_{L 1}^{2}+2 y_{L t}^{2}\left[\log \left(\frac{2 m_{1}^{2}}{v^{2} y_{L t}^{2}}\right)-1\right]\right\}
$$



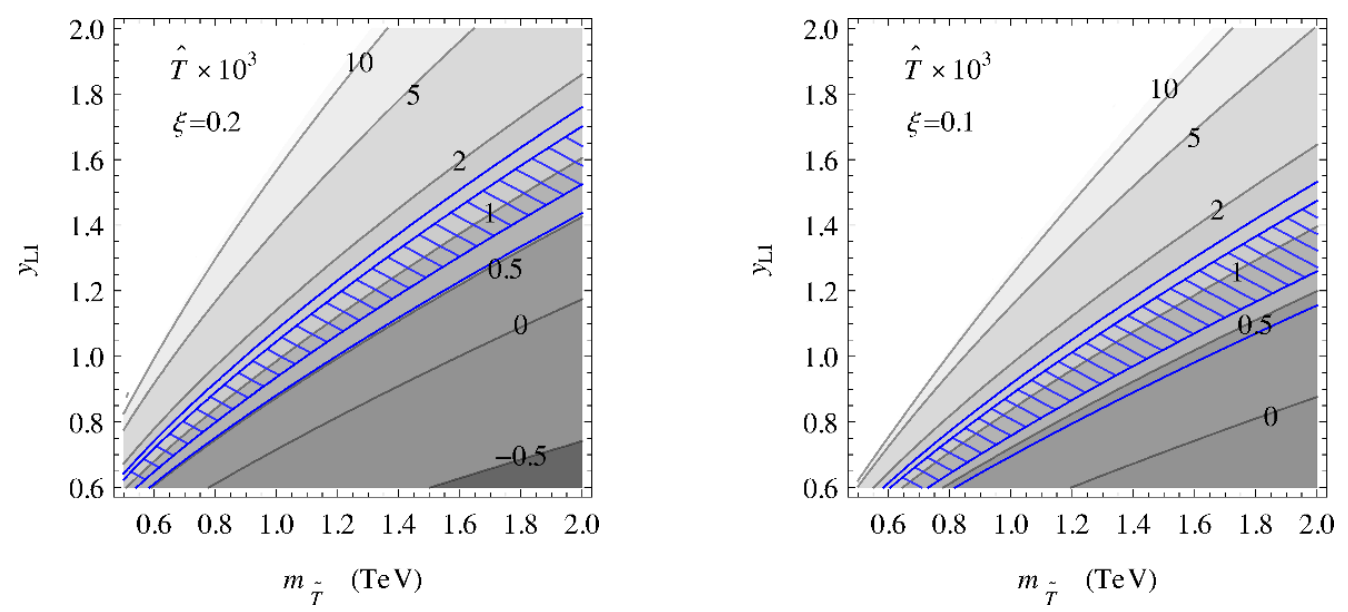

Figure 14. Corrections to the $\widehat{T}$ parameter as a function of the mass of the top partners and of the $q_{L}$ compositeness. The result corresponds to the scenario with a totally composite $t_{R}$ with only a light singlet. The compositeness scale has been fixed to $\xi=0.2$ in the left panel and $\xi=0.1$ in the right one. The solid blue contours give the regions that pass the constraints on the oblique parameters at the $68 \%$ and $95 \%$ confidence level.

By comparing this expression with the result in eq. (5.5) we find the same relation we obtained in subsection 4.1: $\Delta \widehat{T}_{\text {ferm }}=3 \delta g_{b_{L}}$. The values of $\widehat{T}$ compatible with the bounds $\left(0 \lesssim \widehat{T} \lesssim 2 \cdot 10^{-3}\right)$ imply a moderate positive shift in $\delta g_{b_{L}}$. This shift slightly worsens the agreement with the experimental data with respect to the SM.

The case of a light 4-plet. The second simplified model we consider is the effective theory with only a light 4-plet. As can be seen from eqs. (5.1) and (5.2), in this case the low-energy Lagrangian contains 4 free parameters: the elementary-composite mixings, the 4 -plet mass and the coefficient of the $d$-symbol term, $c_{t}$. As we will see, the $d$-symbol term can sizably affect the corrections to the EW observables. Its presence makes the properties of the model quite different from the ones found in the case with an elementary $t_{R}$ (compare subsection 4.2). Moreover, as was pointed out in the analysis of ref. [40], the $d$-symbol term can also play an important role for collider phenomenology.

In addition to the corrections from the Higgs non-linear dynamics and the UV tree-level shift, the $\widehat{S}$ parameter receives a logarithmically enhanced contributions from fermion loops:

$$
\Delta \widehat{S}_{\mathrm{ferm}}^{\mathrm{div}}=\frac{g^{2}}{8 \pi^{2}}\left(1-c_{t}^{2}\right) \xi \log \left(\frac{m_{*}^{2}}{m_{4}^{2}}\right)
$$

If $c_{t}$ is not close to 1 , this shift can be sizable and can induce stringent constraints on the compositeness scale $\xi$.

The contributions to the $\widehat{T}$ parameter coming from fermion loops at leading order in $v / f$ are given by

$$
\Delta \widehat{T}_{\text {ferm }}=-\frac{\xi}{32 \pi^{2}} \frac{y_{L 4} f^{2}}{m_{4}^{2}}\left\{3 c_{t}^{2} y_{L 4}\left(y_{L 4}^{2}-4 y_{L t}^{2}\right)+y_{L 4}^{2}\left(y_{L 4}-3 \sqrt{2} c_{t} y_{L t}\right)\right.
$$



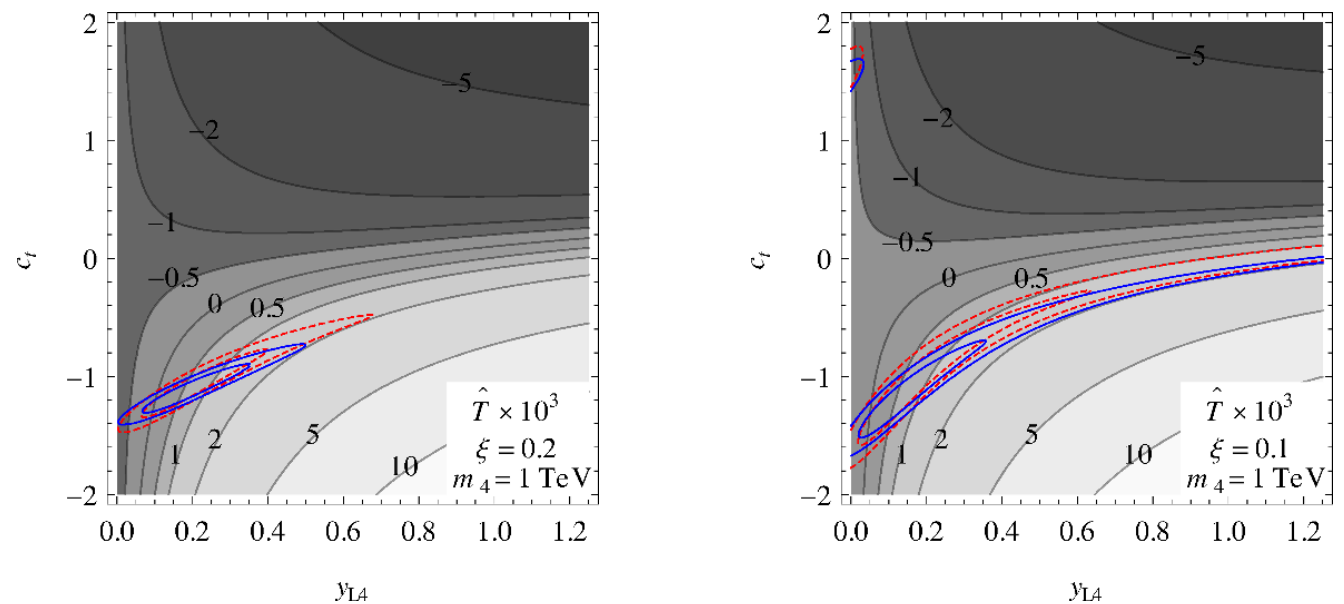

Figure 15. Corrections to the $\widehat{T}$ parameter as a function of the $y_{L 4}$ mixing and of $c_{t}$. The result corresponds to the scenario with a totally composite $t_{R}$ with only a light 4-plet with mass $m_{4}=1 \mathrm{TeV}$. The compositeness scale has been fixed to $\xi=0.2$ in the left panel and $\xi=0.1 \mathrm{in}$ the right one. The solid blue contours give the regions that pass the constraints on the oblique parameters at the $68 \%$ and $95 \%$ confidence level. The dashed red lines show how the bounds are modified if we assume a $25 \%$ reduction in $\widehat{S}$.

$$
\left.-3 y_{L t}^{2}\left(y_{L 4}-4 \sqrt{2} c_{t} y_{L t}\right)\left[\log \left(\frac{2 m_{4}^{2}}{v^{2} y_{L t}^{2}}\right)-1\right]\right\} .
$$

Notice that the terms related to the $d$-symbol operator come with accidentally large coefficients, thus even a relatively small value of $c_{t}$ can drastically modify the result. In figure 15 we show the total correction to $\widehat{T}$ as a function of $y_{L 4}$ and $c_{t}$ for a fixed value of the 4 -plet mass, $m_{4}=1 \mathrm{TeV}$. One can see that a positive correction to the $\widehat{T}$ parameter is possible, but requires a sign correlation between $y_{L 4}$ and $c_{t} .{ }^{18} \mathrm{In}$ the plots we also show the regions compatible with the constraints on the oblique parameters. The parameter space regions with better agreement with the EW data are the ones with $c_{t} \sim-1$, in which the logarithmically enhanced shift in $\widehat{S}$ is partially cancelled.

The corrections to the $Z \bar{b}_{L} b_{L}$ vertex are given at the leading order in $v / f$ by

$$
\begin{aligned}
\delta g_{b_{L}}= & -\frac{\xi}{64 \pi^{2}} \frac{m_{4}^{2} y_{L 4} y_{L t}^{2} f^{2}}{\left(m_{4}^{2}+y_{L 4}^{2}\right)^{2}}\left[2 y_{L 4}-\sqrt{2} c_{t} y_{L t}\right. \\
& \left.+\left(2 y_{L 4}-\sqrt{2} c_{t} y_{L t}+\frac{y_{L 4} y_{L t}^{2} f^{2}}{2\left(m_{4}^{2}+y_{L 4}^{2} f^{2}\right)}\right) \log \left(\frac{v^{2} m_{4}^{2} y_{L t}^{2}}{2\left(m_{4}^{2}+y_{L 4}^{2} f^{2}\right)^{2}}\right)\right] .
\end{aligned}
$$

The above formula contains only the corrections coming from the lowest order terms in the effective Lagrangian without the contributions from 4 -fermion operators. As can be seen from the numerical result in the left panel of figure 16, the sign of $\delta g_{b_{L}}$ has some correlation with the sign of $\widehat{T}$. The size of the corrections to the $Z \bar{b}_{L} b_{L}$ vertex is however typically one order of magnitude smaller than the one in $\widehat{T}$. The points compatible with the constraints on the oblique EW parameters have $\delta g_{b_{L}}$ in the range $0 \lesssim \delta g_{b_{L}} \lesssim 0.5 \cdot 10^{-3}$.

\footnotetext{
${ }^{18}$ Notice that the Lagrangian is invariant under the transformation $y_{L 4} \rightarrow-y_{L 4}$ and $c_{t} \rightarrow-c_{t}$.
} 

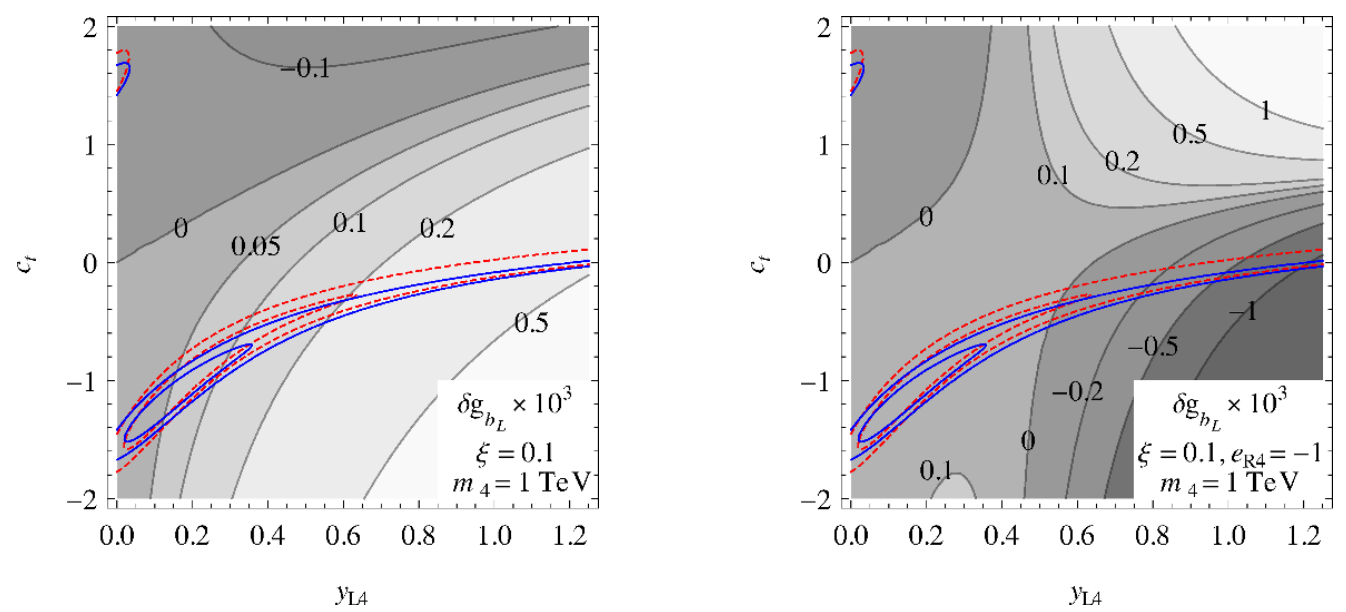

Figure 16. Corrections to the $Z \bar{b}_{L} b_{L}$ vertex as a function of the $y_{L 4}$ mixing and of $c_{t}$. The results correspond to the scenario with a totally composite $t_{R}$ with only a light 4-plet with mass $m_{4}=1 \mathrm{TeV}$. The compositeness scale has been fixed by $\xi=0.1$. In the left panel we neglected the contributions from 4 -fermion operators, while in the right panel we included the corrections due to the operator in eq. (4.19) with $e_{R 4}=-1$.

The corrections to the $Z \bar{b}_{L} b_{L}$ vertex can of course be modified if 4 -fermion interactions are present in the effective Lagrangian. In particular logarithmically divergent contributions can be induced by operators of the form given in eq. (3.18). As an example we will show how the previous result for $\delta g_{b_{L}}$ is modified by the operator given in eq. (4.19). In this case the following additional contribution arises:

$$
\delta g_{b_{L}}=\frac{e_{R 4}}{32 \pi^{2}} \xi \frac{y_{L 4}^{2} f^{2}}{m_{4}^{2}+y_{L 4}^{2} f^{2}} y_{L 4}\left(y_{L 4}-\sqrt{2} c_{t} y_{L t}\right) \log \left(\frac{m_{*}^{2}}{m_{4}^{2}}\right),
$$

In the right panel of figure 16 we show the numerical result for $\delta g_{b_{L}}$ including the extra contribution in eq. (5.10) for $e_{R 4}=-1$. In the region with sizable values for $y_{L 4}$ the new contribution dominates and can induce a negative shift in $\delta g_{b_{L}}$, which would improve the compatibility with the experimental measurements.

\section{Corrections to the top couplings}

So far we devoted our attention to the oblique EW parameters and the bottom couplings. The tight experimental bounds on these observables do not allow for large deviations from the SM predictions and lead to strong bounds on the new physics effects. Another class of observables, in particular the ones related to the top quark, are instead less constrained from the present data which allow sizable deviation from the SM. Large corrections to the top couplings are naturally predicted in the scenarios with partial compositeness due to the strong mixing of the third generation quarks with the composite dynamics. Notice that the $P_{L R}$ invariance, which suppresses the corrections to the $Z \bar{b}_{L} b_{L}$ vertex, does not protect the couplings of the top quark. Thus big tree-level contributions can be generated which 
could be eventually tested at the LHC. The aim of this section is to determine the size of the distortion of the top couplings to the $Z$ and to the $W$ bosons.

The top coupling to the $Z$ boson are described by the following effective Lagrangian

$$
\mathcal{L}^{Z}=\frac{g}{c_{w}} Z_{\mu} \bar{t} \gamma^{\mu}\left[\left(g_{t_{L}}^{\mathrm{SM}}+\delta g_{t_{L}}\right) P_{L}+\left(g_{t_{R}}^{\mathrm{SM}}+\delta g_{t_{R}}\right) P_{R}\right] t
$$

where $g^{\mathrm{SM}}$ denote the SM couplings and $\delta g$ correspond to the new physics contributions. In the above formula $P_{L, R}$ are the left and right chiral projectors. The tree-level values of the SM couplings are given by

$$
g_{t_{L}}^{\mathrm{SM}}=\frac{1}{2}-\frac{2}{3} s_{w}^{2}, \quad g_{t_{R}}^{\mathrm{SM}}=-\frac{2}{3} s_{w}^{2} .
$$

The couplings of the left-handed top component with the charged $W$ boson are related to the $V_{t b}$ element of the CKM matrix. We will parametrize the new physics contributions as $V_{t b}=1+\delta V_{t b}$. The current LHC results already put a constraint on the new physics contribution at the $10 \%$ level: $V_{t b}=1.020 \pm 0.046$ (meas.) \pm 0.017 (theor.) [83]. As we will see, the bounds on the models coming from this measurement are still weaker than the ones coming from the EW precision data.

\subsection{A relation between $\delta g_{t_{L}}$ and $\delta V_{t b}$}

Before discussing the results in the explicit models we considered in this paper, we rederive a general relation that links the deviations in the $Z \bar{t}_{L} t_{L}$ vertex to the corrections to $V_{t b}$ as already noticed in refs. [84-86]. In the effective Lagrangian describing the Higgs doublet and the SM fields only two dimension-six operators contribute to the corrections to the $t_{L}$ couplings $[19,78,84,87]$ :

$$
\mathcal{L}=i \frac{c_{H q}}{f^{2}}\left(\bar{q}_{L} \gamma^{\mu} q_{L}\right)\left(H^{\dagger} \overleftrightarrow{D_{\mu}} H\right)+i \frac{c_{H q}^{\prime}}{f^{2}}\left(\bar{q}_{L} \sigma^{i} \gamma^{\mu} q_{L}\right)\left(H^{\dagger} \sigma^{i} \overleftrightarrow{D_{\mu}} H\right)
$$

A combination of the two operators in eq. (6.3) is strongly constrained by the experimental bound on the corrections to the $Z \bar{b}_{L} b_{L}$ vertex. Notice that, in the models we considered in our analysis, the corrections to $g_{b_{L}}$ exactly vanish at tree level thanks to the $P_{L R}$ symmetry. The condition of vanishing corrections to the $Z \bar{b}_{L} b_{L}$ coupling implies the relation $c_{H q}^{\prime}=-c_{H q}[81,82,88]$. Using this relation we find that the operators in eq. (6.3) give rise to the following interactions of the top quark with the EW gauge bosons:

$$
\mathcal{L} \supset 2 c_{H q} v^{2}\left[\frac{g}{c_{w}} \bar{t}_{L} Z^{\mu} \gamma_{\mu} t_{L}+\frac{g}{2}\left(\bar{t}_{L}\left(W_{\mu}^{1}-i W_{\mu}^{2}\right) \gamma^{\mu} b_{L}+\text { h.c. }\right)\right] .
$$

From this equation we can easily conclude that the leading corrections to the $Z \bar{t}_{L} t_{L}$ vertex and to the $V_{t b}$ matrix element satisfy the relation

$$
\delta g_{t_{L}}=\delta V_{t b} .
$$

Notice that the above result holds only at order $v^{2} / f^{2}$. The subleading terms, as for instance the dimension-eight operators, can generate independent corrections to $g_{t_{L}}$ and $V_{t b}$.

It is important to stress that this analysis is valid as far as we can neglect the corrections to the $Z \bar{b}_{L} b_{L}$ vertex with respect to the corrections to the top couplings. Thus the result in eq. (6.5) is true in general and not only in the composite Higgs scenarios. 

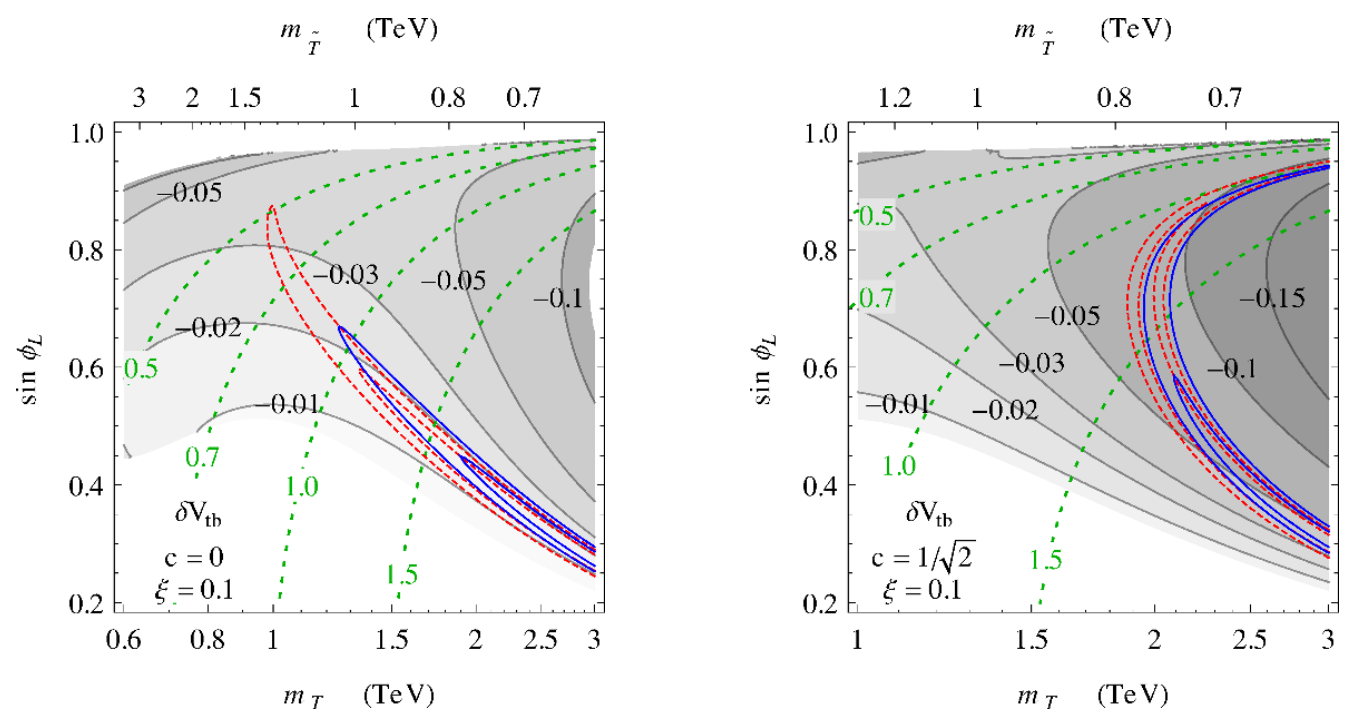

Figure 17. Corrections to the $V_{t b}$ matrix element in the complete models with $c=0$ (left panel) and $c=1 / \sqrt{2}$ (right panel) for $\xi=0.1$. The configurations correspond to the ones of the left plot of figure 10 for the case $c=0$ and of the right plot of figure 12 for the case $c=1 / \sqrt{2}$.

\subsection{The case of an elementary $t_{R}$}

As a first class of models we consider the scenarios with an elementary $t_{R}$. The corrections to the $t_{L}$ couplings at leading order in $v / f$ are given by

$$
\delta g_{t_{L}}=\delta V_{t b}=-\frac{\xi}{4} \frac{f^{2}}{m_{4}^{2}+y_{L 4}^{2} f^{2}}\left[\left(\frac{m_{4} m_{1} y_{L 1}+y_{L 4} y_{R 4} y_{R 1} f^{2}}{m_{1}^{2}+y_{R 1}^{2} f^{2}}-\sqrt{2} c y_{L 4}\right)^{2}+\left(1-2 c^{2}\right) y_{L 4}^{2}\right] \text {. }
$$

This explicit result is in agreement with the relation derived in the previous subsection (see eq. (6.5)). We also verified that at order $(v / f)^{4}$ the corrections to $g_{t_{L}}$ and $V_{t b}$ do not coincide.

The coupling of the $t_{R}$ with the $Z$ boson is modified as well. The leading corrections take the form

$$
\delta g_{t_{R}}=\frac{\xi}{4} \frac{f^{2}}{m_{1}^{2}+y_{R 1}^{2} f^{2}}\left[\left(\frac{m_{4} m_{1} y_{R 4}+y_{L 4} y_{L 1} y_{R 1} f^{2}}{m_{4}^{2}+y_{L 4}^{2} f^{2}}-\sqrt{2} c y_{R 1}\right)^{2}-\left(\frac{m_{1} y_{R 4}}{m_{4}}-\sqrt{2} c y_{R 1}\right)^{2}\right] .
$$

As explicit numerical examples we show in figure 17 the distortion of the $V_{t b}$ matrix element in the complete models with $c=0$ and $c=1 / \sqrt{2}$ (see subsection 4.3). In the case with $c=0$, the configurations allowed by the constraints on the oblique EW parameters have small corrections to $V_{t b},-0.03 \lesssim \delta V_{t b} \lesssim 0$, which are below the present experimental sensitivity. On the contrary, in the model with $c=1 / \sqrt{2}$, the corrections to $V_{t b}$ can be sizable, $-0.12 \lesssim \delta V_{t b} \lesssim-0.03$, and the current bounds can already exclude a corner of the parameter space allowed by the EW precision data. In our numerical analysis we also found that, in the realistic regions of the parameter space, the deviations in the $t_{R}$ couplings are always 
small, $\delta g_{t_{R}} \lesssim 0.01$. Moreover we checked numerically that the correlation between $\delta g_{t_{L}}$ and $\delta V_{t b}$ is always well verified and the deviations from eq. (6.5) are of order $\xi$ as expected.

To conclude the analysis of the top couplings in the models with an elementary $t_{R}$, it is interesting to consider the simplified cases with only one light composite multiplet. In the limit with only a light singlet we find

$$
\delta g_{t_{L}}=\delta V_{t b}=-\frac{\xi}{4} \frac{m_{1}^{2} y_{L 1}^{2} f^{2}}{\left(m_{1}^{2}+y_{R 1}^{2} f^{2}\right)^{2}}, \quad \delta g_{t_{R}}=0 .
$$

This shows that the corrections to the $t_{L}$ couplings are suppressed in the parameter space region with a sizable $t_{R}$ compositeness $\left(y_{R 1} f>m_{1}\right.$ and $\left.y_{R 1}>y_{L 1}\right)$. The corrections to $g_{t_{R}}$ vanish in this case because the $t_{R}$ can only mix with composite states with the same coupling to the $Z$ boson.

In the case with only a light 4 -plet we obtain the following results

$$
\delta g_{t_{L}}=\delta V_{t b}=-\frac{\xi}{4} \frac{y_{L 4}^{2} f^{2}}{m_{4}^{2}+y_{L 4}^{2} f^{2}}, \quad \delta g_{t_{R}}=-\frac{\xi}{4} \frac{y_{L 4}^{2} y_{R 4}^{2} f^{2}}{m_{4}^{2}+y_{L 4}^{2} f^{2}}\left(\frac{f^{2}}{m_{4}^{2}}+\frac{f^{2}}{m_{4}^{2}+y_{L 4}^{2} f^{2}}\right)
$$

In this case the experimental bounds on $V_{t b}$ can be used to put an upper bound on the $t_{L}$ compositeness. Notice that the mixing of the $t_{R}$ does not break the $P_{L R}$ symmetry. The $g_{t_{R}}$ coupling, however, can receive tree-level corrections through the mixing between the elementary $t_{R}$ and composite resonances with different quantum numbers, which is induced by the non-zero top mass. This origin explains why the prefactor in the expression for $\delta g_{t_{R}}$ is proportional to the square of the top Yukawa (see eq. (4.8)). The correction to $g_{t_{R}}$ is enhanced if the top partners are light.

\subsection{The case of a composite $t_{R}$}

We now consider the scenarios with a totally composite $t_{R}$. The leading corrections to the $V_{t b}$ matrix element and to the top couplings to the $Z$ boson are given by

$$
\delta g_{t_{L}}=\delta V_{t b}=-\frac{\xi}{4} \frac{f^{2}}{m_{4}^{2}+y_{L 4}^{2} f^{2}}\left[\left(\frac{m_{4} y_{L 1}}{m_{1}}-\sqrt{2} c_{L} y_{L 4}\right)^{2}+\left(1-2 c_{L}^{2}\right) y_{L 4}^{2}\right],
$$

and

$$
\delta g_{t_{R}}=\frac{\xi}{4} \frac{y_{L 4} y_{L t} f^{2}}{\left(m_{4}^{2}+y_{L 4}^{2} f^{2}\right)^{2}}\left[y_{L 4} y_{L t} f^{2}-2 \sqrt{2} c_{t}\left(m_{4}^{2}+y_{L 4}^{2} f^{2}\right)\right]
$$

In the limits with only one light multiplet the expressions in eqs. (6.10) and (6.11) can be drastically simplified. If only a light singlet is present in the effective theory we find:

$$
\delta g_{t_{L}}=\delta V_{t b}=-\frac{\xi}{4} \frac{y_{L 1}^{2} f^{2}}{m_{1}^{2}}, \quad \delta g_{t_{R}}=0 .
$$

In this case the corrections to the $Z \bar{t}_{R} t_{R}$ coupling are negligible, while the $V_{t b}$ matrix element and the $Z \bar{t}_{L} t_{L}$ vertex can become large if the composite singlet is light. 
In the model with only a light composite 4-plet the corrections to the top couplings become

$$
\delta g_{t_{L}}=\delta V_{t b}=-\frac{\xi}{4} \frac{y_{L 4}^{2} f^{2}}{m_{4}^{2}+y_{L 4}^{2} f^{2}}, \quad \delta g_{t_{R}}=\frac{\xi}{4} \frac{y_{L 4} y_{L t} f^{2}}{\left(m_{4}^{2}+y_{L 4}^{2} f^{2}\right)^{2}}\left[y_{L 4} y_{L t} f^{2}-2 \sqrt{2} c_{t}\left(m_{4}^{2}+y_{L 4}^{2} f^{2}\right)\right] .
$$

Analogously to the case with an elementary $t_{R}$, the corrections to the $V_{t b}$ matrix element can be used to put an upper bound on the degree of compositeness of the elementary doublet $q_{L}$.

\section{Conclusions}

In this work we studied the corrections to the EW observables that arise in composite Higgs scenarios due to the presence of fermionic resonances. In realistic models light composite fermions are typically predicted and this motivated the use of an effective field theory approach for our analysis. For definiteness we focused our attention on the minimal composite Higgs realization based on the symmetry structure $\mathrm{SO}(5) / \mathrm{SO}(4)$. Within this framework we considered a general parametrization of the case in which the elementary SM fermions are mixed with operators in the fundamental representation of the global $\mathrm{SO}(5)$ group. We included in our effective Lagrangian one level of composite fermionic resonances which correspond to a 4-plet and a singlet under the unbroken SO(4) symmetry.

We quantified the relevance of the fermionic contribution to the deviation of the precision electroweak observables. In particular we focused on the oblique electroweak parameters, $\widehat{S}$ and $\widehat{T}$, and on the $Z \bar{b}_{L} b_{L}$ coupling, which are very well determined experimentally and can be used to put tight constraints on new physics effects.

One interesting result is the identification of a new parametrically enhanced contribution to the $\widehat{S}$ parameter. This effect is entirely generated by the composite dynamics and appears if light composite fermions (in particular $\mathrm{SO}(4)$ 4-plets) are present in the spectrum. The origin of the new enhanced contribution can easily be understood from an effective field theory point of view. The non-renormalizable Higgs interactions due to the non-linear $\sigma$-model dynamics induce new logarithmically divergent diagrams and generate a running of the two dimension- 6 operators, $\mathcal{O}_{W, B}$, which contribute to the $\widehat{S}$ parameter. This effect is calculable in the effective theory and its size turns out to be comparable or even larger than the tree-level shift given by the heavy gauge resonances.

In minimal scenarios with a light 4-plet $\left(m_{4} \lesssim 1 \mathrm{TeV}\right)$, the constraints on the $\widehat{S}$ parameter imply a tight bound on the compositeness scale $\xi \lesssim 0.1$, which corresponds to $f \gtrsim 750 \mathrm{GeV}$ (see figure 3). This bound can be relaxed if additional light states are present in the spectrum (for instance a singlet). Cancelling the 4-plet contribution, however, seems possible only at the price of some additional tuning.

Another consequence of the presence of logarithmic divergence in $\widehat{S}$ is the fact that the UV dynamics does not necessarily decouple and can generate non-negligible finite corrections. We discussed an example of this effect in one explicit model, but we did not systematically investigate this aspect. We leave this analysis for future work.

Differently from $\widehat{S}$, the $\widehat{T}$ parameter is finite in our scenario thanks to the protection coming from the custodial symmetry. The corrections coming from the composite sector 
are thus dominated by the contributions of the lightest composite states and can be reliably computed in our effective field theory. This allows us to use the $\widehat{T}$ parameter to put robust bounds on the parameter space of the composite Higgs models.

We found that a positive shift in $\widehat{T}$, which is typically needed to satisfy the constraints on the oblique parameters, can be easily generated by the fermion loops. In the standard scenarios, in which the $t_{R}$ is a partially elementary state, obtaining a positive correction to $\widehat{T}$ requires the presence of a relatively light singlet. In configurations with only a light 4-plet the corrections are instead always negative. On the contrary, in the alternative scenarios in which the $t_{R}$ is a completely composite state, a positive contribution to $\widehat{T}$ can be obtained also in the configurations with only a light 4 -plet. This can be done at the price of a mild correlation among the parameters (see figure 15).

The third precision observable we considered is the $Z \bar{b}_{L} b_{L}$ coupling. In this case, powercounting arguments show that the composite resonances contributions can be logarithmically divergent. We found however that, if only the operators with the lowest dimension are included in the effective Lagrangian, a selection rule forbids the appearance of divergent contributions and makes the corrections to the $g_{b_{L}}$ coupling finite. This is no longer true if higher dimensional operators and in particular 4-fermion interactions are present in the effective theory. In this case, if a light 4-plet is included in the theory, a logarithmically enhanced correction to $g_{b_{L}}$ can be generated. Moreover, as in the case of the $\widehat{S}$ parameter, the UV dynamics typically does not decouple and can generate sizable corrections.

If only the lowest-dimensional operators are included in the effective Lagrangian, the corrections to the $Z \bar{b}_{L} b_{L}$ vertex tend to be correlated to the corrections to $\widehat{T}$. In particular a sizable positive shift in $\widehat{T}$ usually corresponds to a positive contribution to $\delta g_{b_{L}}$, which is disfavored by the current experimental bounds. Higher-dimensional operators, which are typically generated by the composite dynamics, can however induce large contributions to the $Z \bar{b}_{L} b_{L}$ coupling and remove the correlation with $\widehat{T}$.

Finally we analyzed the corrections to the top EW gauge couplings. In the composite Higgs scenarios we considered these couplings can receive large tree-level distortions due to the sizable degree of compositeness of the top. We found that the deviations of the $Z \bar{t}_{L} t_{L}$ vertex are strongly correlated with the corrections to the $W t_{L} b_{L}$ coupling. Stringent bounds on the deviations of the $V_{t b}$ matrix element would therefore strongly disfavor the presence of large corrections to the $Z$ coupling.

The constraints on the model coming from the current measurement of the $V_{t b}$ matrix element are typically weaker than the ones from the EW precision data and can become competitive with them only in a small region of the parameter space. For a moderate amount of tuning, $\xi=0.1$, the corrections to the $V_{t b}$ matrix element can be of order $5 \%$ and the corrections to the $Z \bar{t}_{L} t_{L}$ of order $10 \%$.

\section{Acknowledgments}

We thank E. Furlan, R. Rattazzi, S. Rychkov, M. Serone and M. Trott for useful discussions. We also thank A. Wulzer for many suggestions and comments about the manuscript. O. M. and G. P. acknowledge the Galileo Galilei Institute in Florence for hospitality during 
the completion of this work. The work of C. G. and O. M. is supported by the European Programme Unification in the LHC Era, contract PITN-GA-2009-237920 (UNILHC). C. G. is also supported by the Spanish Ministry MICNN under contract FPA2010-17747. This research has been partly funded by the European Commission under the ERC Advanced Grant 226371 MassTeV.

\section{A The CCWZ notation}

In this appendix we define our notation for the $\mathrm{SO}(5)$ algebra and for the CCWZ operators. For most of our definitions we follow the notation of ref. [40].

\section{The SO(5) algebra and the Goldstones}

A useful basis for the $\mathrm{SO}(5)$ generators, which shows explicitly the $\mathrm{SO}(4)$ subgroup, is given by

$$
\begin{aligned}
\left(T_{L, R}^{\alpha}\right)_{I J} & =-\frac{i}{2}\left[\frac{1}{2} \varepsilon^{\alpha \beta \gamma}\left(\delta_{I}^{\beta} \delta_{J}^{\gamma}-\delta_{J}^{\beta} \delta_{I}^{\gamma}\right) \pm\left(\delta_{I}^{\alpha} \delta_{J}^{4}-\delta_{J}^{\alpha} \delta_{I}^{4}\right)\right] \\
T_{I J}^{i} & =-\frac{i}{\sqrt{2}}\left(\delta_{I}^{i} \delta_{J}^{5}-\delta_{J}^{i} \delta_{I}^{5}\right),
\end{aligned}
$$

where $T_{L, R}^{\alpha}(\alpha=1,2,3)$ correspond to the $\mathrm{SO}(4) \simeq \mathrm{SU}(2)_{L} \times \mathrm{SU}(2)_{R}$ generators, $T^{i}$ $(i=1, \ldots, 4)$ are the generators of the coset $\mathrm{SO}(5) / \mathrm{SO}(4)$ and the indices $I, J$ take the values $1, \ldots, 5$. We chose to normalize the generators in eqs. (A.1) and (A.2) such that $\operatorname{Tr}\left[T^{A}, T^{B}\right]=\delta^{A B}$. With this normalization the $\mathrm{SU}(2)_{L, R}$ generators satisfy the usual commutation relations

$$
\left[T_{L, R}^{\alpha}, T_{L, R}^{\beta}\right]=i \varepsilon^{\alpha \beta \gamma} T_{L, R}^{\gamma} .
$$

The Goldstone matrix for the coset $\mathrm{SO}(5) / \mathrm{SO}(4)$ is given by

$$
U=\exp \left[i \frac{\sqrt{2}}{f_{\pi}} \Pi_{i} T^{i}\right]=\left(\begin{array}{cc}
I_{4 \times 4}-\frac{\vec{\Pi} \vec{\Pi}^{t}}{\Pi^{2}}\left(1-\cos \frac{\Pi}{f}\right) & \frac{\vec{\Pi}}{\Pi} \sin \frac{\Pi}{f} \\
-\frac{\vec{\Pi}^{t}}{\Pi} \sin \frac{\Pi}{f} & \cos \frac{\Pi}{f}
\end{array}\right),
$$

where we defined $\Pi^{2} \equiv \vec{\Pi}^{t} \vec{\Pi}$. Under an SO(5) transformation, $g$, the Goldstones transform according to the standard relation

$$
U(\Pi) \rightarrow U\left(\Pi^{(g)}\right)=g \cdot U(\Pi) \cdot h^{t}(\Pi ; g),
$$

where $h(\Pi ; g)$ is an element of the $\mathrm{SO}(4)$ subgroup:

$$
h=\left(\begin{array}{cc}
h_{4} & 0 \\
0 & 1
\end{array}\right)
$$

Under the unbroken SO(4) symmetry the Goldstones transform linearly: $\Pi^{i} \rightarrow\left(h_{4}\right)_{j}^{i} \Pi^{j}$. 
The standard Higgs doublet $H=\left(h_{u}, h_{d}\right)$ is related to the $\Pi 4$-plet as

$$
\Pi=\frac{1}{\sqrt{2}}\left(\begin{array}{c}
-i\left(h_{u}-h_{u}^{\dagger}\right) \\
h_{u}+h_{u}^{\dagger} \\
i\left(h_{d}-h_{d}^{\dagger}\right) \\
h_{d}+h_{d}^{\dagger}
\end{array}\right) .
$$

The physical Higgs $\rho$ can be obtained adopting the unitary gauge in which the Higgs doublet reads

$$
h_{d} \equiv \frac{h}{\sqrt{2}}=\frac{\langle h\rangle+\rho}{\sqrt{2}}, \quad h_{u}=0 .
$$

In this gauge the Goldstone matrix takes the simple form

$$
U=\left(\begin{array}{ccccc}
1 & 0 & 0 & 0 & 0 \\
0 & 1 & 0 & 0 & 0 \\
0 & 0 & 1 & 0 & 0 \\
0 & 0 & 0 & \cos \frac{h}{f} & \sin \frac{h}{f} \\
0 & 0 & 0 & -\sin \frac{h}{f} & \cos \frac{h}{f}
\end{array}\right) .
$$

\section{The CCWZ operators}

In order to define the $e_{\mu}$ and $d_{\mu}$ CCWZ symbols it is useful to describe the elementary gauge bosons in an $\mathrm{SO}(5)$ notation. The $\mathrm{SM}$ vector fields are introduced in the theory by weakly gauging the $\mathrm{SU}(2)_{L} \times \mathrm{U}(1)_{R 3}$ subgroup of $\mathrm{SO}(4)$ and their embedding is given explicitly by

$A_{\mu}=\frac{g}{\sqrt{2}} W_{\mu}^{+}\left(T_{L}^{1}+i T_{L}^{2}\right)+\frac{g}{\sqrt{2}} W_{\mu}^{-}\left(T_{L}^{1}-i T_{L}^{2}\right)+g\left(c_{w} Z_{\mu}+s_{w} A_{\mu}\right) T_{L}^{3}+g^{\prime}\left(c_{w} A_{\mu}-s_{w} Z_{\mu}\right) T_{R}^{3}$,

where $g$ and $g^{\prime}$ are the gauge coupling corresponding to the $\mathrm{SU}(2)_{L}$ and $\mathrm{U}(1)_{Y}$ subgroups, while $c_{w}$ and $s_{w}$ are the cosine and sine of the weak mixing angle, $\tan \theta_{w}=g^{\prime} / g$.

To define the CCWZ symbols we can start from the following quantity

$$
\bar{A}_{\mu} \equiv A_{\mu}^{\left(U^{t}\right)}=U^{t}\left[A_{\mu}+i \partial_{\mu}\right] U,
$$

and we can define $e_{\mu}$ and $d_{\mu}$ as the coefficient of the decomposition of $\bar{A}_{\mu}$ in terms of broken and unbroken $\mathrm{SO}(5)$ generators:

$$
\bar{A}_{\mu}=-d_{\mu}^{i} T^{i}-e_{\mu}^{a} T^{a} .
$$

It is not difficult to prove that the $e$ and $d$ symbols transform under $\mathrm{SO}(5)$ as

$$
e_{\mu} \equiv e_{\mu}^{a} t^{a} \rightarrow h_{4}\left[e_{\mu}-i \partial_{\mu}\right] h_{4}^{t} \quad \text { and } \quad d_{\mu}^{i} \rightarrow\left(h_{4}\right)_{j}^{i} d_{\mu}^{j},
$$

where we denoted by $t^{a}$ the $\mathrm{SO}(4)$ generators in a $4 \times 4$ matrix form.

Using the embedding of the gauge fields given in eq. (A.10) we get the explicit expressions

$$
d_{\mu}^{i}=\sqrt{2}\left(\frac{1}{f}-\frac{\sin \Pi / f}{\Pi}\right) \frac{\vec{\Pi} \cdot \nabla_{\mu} \vec{\Pi}}{\Pi^{2}} \Pi^{i}+\sqrt{2} \frac{\sin \Pi / f}{\Pi} \nabla_{\mu} \Pi^{i}
$$




$$
e_{\mu}^{a}=-A_{\mu}^{a}+4 i \frac{\sin ^{2}(\Pi / 2 f)}{\Pi^{2}} \vec{\Pi}^{t} t^{a} \nabla_{\mu} \vec{\Pi}
$$

where $\nabla_{\mu} \Pi$ is defined as

$$
\nabla_{\mu} \Pi^{i}=\partial_{\mu} \Pi^{i}-i A_{\mu}^{a}\left(t^{a}\right)_{j}^{i} \Pi^{j} .
$$

The expressions for the $d_{\mu}$ and $e_{\mu}$ symbols in the unitary gauge are given by

$$
d_{\mu}=-\frac{g}{\sqrt{2}} \sin \frac{h}{f}\left(\begin{array}{c}
W_{\mu}^{1} \\
W_{\mu}^{2} \\
\frac{1}{c_{w}} Z_{\mu} \\
-\frac{2}{g f \sin \frac{h}{f}} \partial_{\mu} h
\end{array}\right)
$$

and

$$
e_{\mu}=\frac{i g}{2}\left(\begin{array}{cccc}
0 & 2 s_{w} A_{\mu}+\frac{1-2 s_{w}^{2}}{c_{w}} Z_{\mu} & -W_{\mu}^{2} & W_{\mu}^{1} \cos \frac{h}{f} \\
-2 s_{w} A_{\mu}-\frac{1-2 s_{w}^{2}}{c_{w}} Z_{\mu} & 0 & W_{\mu}^{1} & W_{\mu}^{2} \cos \frac{h}{f} \\
W_{\mu}^{2} & -W_{\mu}^{1} & 0 & \frac{1}{c_{w}} Z_{\mu} \cos \frac{h}{f} \\
-W_{\mu}^{1} \cos \frac{h}{f} & -W_{\mu}^{2} \cos \frac{h}{f} & -\frac{1}{c_{w}} Z_{\mu} \cos \frac{h}{f} & 0
\end{array}\right) .
$$

Using the $d_{\mu}$ symbol we can write the kinetic term for the Goldstones in the form

$$
\mathcal{L}_{\pi}=\frac{f^{2}}{4} d_{\mu}^{i} d_{i}^{\mu}
$$

In the unitary gauge the above expression becomes

$$
\mathcal{L}_{\pi}=\frac{1}{2}(\partial h)^{2}+\frac{g^{2}}{4} f^{2} \sin ^{2} \frac{h}{f}\left(|W|^{2}+\frac{1}{2 c_{w}^{2}} Z^{2}\right) .
$$

From this expression we can extract the mass of the $W$ boson, $m_{w}=(g / 2) f \sin (\langle h\rangle)$ and derive the exact relation between the Higgs VEV and the EW scale $v=246 \mathrm{GeV}$ :

$$
v=f \sin \frac{\langle h\rangle}{f} .
$$

When a gap between the EW scale $v$ and the compositeness scale $f$ exists, such that $v \ll f$, the Higgs VEV and the EW scale can be identified $v \simeq\langle h\rangle$. As it is clear from our analysis the condition $(v / f)^{2} \ll 1$ is required by the EW constraints and we can safely replace the Higgs VEV with $v$ as we did in this paper.

Finally we discuss the introduction of fermions in the CCWZ notation. We included in our effective theory two possible composite multiplets: $\psi_{4}$ which transforms as a 4-plet of $\mathrm{SO}(4)$ and $\psi_{1}$ which is a singlet. Under the non-linearly realized $\mathrm{SO}(5)$ transformations $\psi_{1}$ is invariant, while $\psi_{4}$ transforms as

$$
\psi_{4} \rightarrow h_{4} \cdot \psi_{4} .
$$

The covariant derivative for the singlet is the standard one

$$
D_{\mu} \psi_{1}=\left[\partial_{\mu}-i g^{\prime} X\left(c_{w} A_{\mu}-s_{w} Z_{\mu}\right)\right] \psi_{1},
$$


where $X$ denotes the charge under $\mathrm{U}(1)_{X}$. The covariant derivative of the 4-plet, instead, contains an extra term given by the $e_{\mu}$ symbol:

$$
D_{\mu} \psi_{4}=\left[\partial_{\mu}+i e_{\mu}-i g^{\prime} X\left(c_{w} A_{\mu}-s_{w} Z_{\mu}\right)\right] \psi_{4} .
$$

The presence of the extra term is essential to restore the full $\mathrm{SO}(5)$ invariance.

\section{B Operator analysis for the $Z \bar{b}_{L} b_{L}$ vertex}

In section 3 we presented a general analysis of the one-loop corrections to the $Z \bar{b}_{L} b_{L}$ vertex that are induced by the presence of composite fermion resonances. We found that logarithmically divergent contributions can be present if a light composite 4-plet is present in the spectrum. For simplicity in the main text we did not report rigorous proofs of our statements and we only gave some partial justifications. The aim of this appendix is to present a more rigorous and systematic study based on an operator analysis.

\section{General considerations}

An important feature of our effective Lagrangian is the presence of a $P_{L R}$ symmetry, which is exact in the composite sector and is only broken by the mixing with the elementary states (in particular with the doublet $q_{L}$ ). The $P_{L R}$ symmetry plays an essential role in protecting the $Z \bar{b}_{L} b_{L}$ vertex from large tree-level corrections and it also leads to a reduction of the degree of divergence of the loop contributions. In the following we will take into account the consequences of the $P_{L R}$ invariance through the method of spurions.

As a first step we need to formally restore the global $\mathrm{SO}(5)$ invariance in our effective Lagrangian. For this purpose we assume that the elementary fields transform only under an "elementary" $\mathrm{SU}(2)_{L} \times \mathrm{U}(1)_{Y}$ global group which is independent with respect to the global $\mathrm{SO}(5)$ invariance of the composite sector. In this picture the SM group corresponds to the diagonal combination of the "elementary" and the "composite" groups. The mixing between the elementary and the composite states clearly induces a breaking of the extended global invariance. We can however formally restore the complete global symmetry by promoting the couplings to spurions with non-trivial transformation properties under the "elementary" and the "composite" groups. In our set-up we need two spurions:

i) $\left(\tilde{y}_{L}\right)_{A}^{\alpha}$, which transforms as a doublet $\left(\overline{\mathbf{2}}_{-1 / 6}\right)$ under the "elementary" symmetry (index $\alpha$ ) and belongs to the fundamental representation of $\mathrm{SO}(5)$ with $\mathrm{U}(1)_{X}$ charge $2 / 3$ (index $A$ ). Its physical value is given by

$$
\left\langle\tilde{y}_{L}\right\rangle=\frac{1}{\sqrt{2}}\left(\begin{array}{cc}
0 & i \\
0 & 1 \\
i & 0 \\
-1 & 0 \\
0 & 0
\end{array}\right) .
$$


ii) $\left(\tilde{y}_{R}\right)_{A}$, which is a singlet under the "elementary" group $\left(\mathbf{1}_{-2 / 3}\right)$ and transforms in the fundamental representation of the "composite" group $\left(\mathbf{5}_{2 / 3}\right)$. Its physical value is given by

$$
\left\langle\tilde{y}_{R}\right\rangle=\left(\begin{array}{l}
0 \\
0 \\
0 \\
0 \\
1
\end{array}\right) .
$$

It is important to remark that in our definition the two spurions transform linearly under the $\mathrm{SO}(5)$ "composite" group.

Using the spurions we can rewrite the elementary-composite mixings in a fully invariant form

$$
\begin{aligned}
\mathcal{L}_{\text {mix }}= & y_{L 4} \bar{q}_{L}^{\alpha}\left(\tilde{y}_{L}^{\dagger}\right)_{A}^{\alpha} U_{A i} \psi_{4}^{i}+y_{L 1} \bar{q}_{L}^{\alpha}\left(\tilde{y}_{L}^{\dagger}\right)_{A}^{\alpha} U_{A 5} \psi_{1} \\
& +y_{R 4} \bar{t}_{R}\left(\tilde{y}_{R}^{\dagger}\right)_{A} U_{A i} \psi_{4}^{i}+y_{R 1} \bar{t}_{R}\left(\tilde{y}_{R}^{\dagger}\right)_{A} U_{A 5} \psi_{1}+\text { h.c. }
\end{aligned}
$$

Notice that the two mixings of the $q_{L}$ doublet are associated to the same spurion $\tilde{y}_{L}$ and analogously the $t_{R}$ mixings correspond to the spurion $\tilde{y}_{R}$. From the Lagrangian in eq. (B.3) we can recover the original mixing terms in eq. (2.9) by replacing the spurions with their physical values $\left\langle\tilde{y}_{L, R}\right\rangle$.

We can now identify the building blocks that can be used to construct the operators in our effective theory. One key element is of course the Goldstone matrix $U$. As shown in eq. (A.5), under the $\mathrm{SO}(5)$ group $U$ transforms linearly on one side and non-linearly on the other. We can thus split the Goldstone matrix in two components: $U_{A i}$ whose index $i$ transforms as a CCWZ 4-plet and $U_{A 5}$ which is a singlet. In both cases the index $A$ corresponds to a linear realization of the fundamental representation of $\mathrm{SO}(5)$.

It is also useful to introduce a slight generalization of the covariant derivative. We define it in such a way that it acts on all the indices of a given object, for instance the covariant derivative of the 4-plet Goldstone component is

$$
\left(D_{\mu} U\right)_{A i} \equiv \partial_{\mu} U_{A i}-i\left(A_{\mu} U\right)_{A i}-i\left(U e_{\mu}\right)_{A i} .
$$

For the elementary fermions and the composite resonances the convariant derivative coincides with the one we used so far. It is useful to notice that the covariant derivative of the Goldstone matrix can always be expressed in terms of the $d_{\mu}$ symbol:

$$
\left(D_{\mu} U\right)_{A i}=-U_{A 5} d_{\mu}^{i} \quad \text { and } \quad\left(D_{\mu} U\right)_{A 5}=-U_{A i} d_{\mu}^{i} .
$$

Moreover it is easy to check that the covariant derivative of the spurions vanishes when it is computed on the spurion physical values, $\left\langle D_{\mu} y_{L, R}\right\rangle=0$.

In our analysis, for simplicity, we will consider the limit in which the gauge couplings are sent to zero. This limit is justified by the fact that the largest corrections to the $Z \bar{b}_{L} b_{L}$ vertex come from loops containing the Goldstones and not the transverse gauge field components. Within this approximation, the elementary fermion interactions are 
necessarily mediated by the elementary-composite mixings. This implies that, in classifying the operators that contribute to the $Z \bar{b}_{L} b_{L}$ coupling, we can assume that the elementary fields are always contracted with the $\tilde{y}_{L, R}$ spurions.

To construct the operators that can appear in the effective Lagrangian we can use the following building blocks: ${ }^{19}$

$$
\begin{aligned}
\text { elementary fields: } & q_{L}^{\alpha} \text { and } t_{R} \\
\text { composite fields: } & \psi_{4}^{i} \text { and } \psi_{1} \\
\text { cov. der. of the fermions: } & \left(D_{\mu} q_{L}\right)^{\alpha}, D_{\mu} t_{R},\left(D_{\mu} \psi_{4}\right)^{i} \text { and } D_{\mu} \psi_{1} \\
d_{\mu} \text { symbol: } & d_{\mu}^{i} \\
\text { mixings: } & \left(U^{\dagger} \tilde{y}_{L}\right)_{i, 5}^{\alpha} \text { and }\left(U^{\dagger} \tilde{y}_{R}\right)_{i, 5}
\end{aligned}
$$

Notice that, thanks to the unitarity of the Goldstone matrix, we can always write the spurions in the combinations $U^{\dagger} \tilde{y}_{L, R}$.

\section{Classification of the operators}

We can now analyze the operators that can modify the coupling of the $Z$ boson to the $b_{L}$ with the aim of determining their degree of divergence. This can be easily achieved by classifying the operators in an expansion in the elementary-composite mixings.

To simplify the analysis it is more convenient to work in the basis of the elementary and composite fields and not in the one of the mass eigenstates. The mass eigenstate corresponding to the physical $b_{L}$, which we will denote here by $\widetilde{b}_{L}$, is given by a combination of the elementary $b_{L}$ and of the composite state $B$ contained in the 4-plet $\psi_{4}$ :

$$
\begin{aligned}
b_{L} & =\frac{m_{4}}{\sqrt{m_{4}^{2}+y_{L 4}^{2} f^{2}}} \widetilde{b}_{L}-\frac{y_{L 4} f}{\sqrt{m_{4}^{2}+y_{L 4}^{2} f^{2}}} \widetilde{B}_{L}, \\
B_{L} & =\frac{y_{L 4} f}{\sqrt{m_{4}^{2}+y_{L 4}^{2} f^{2}}} \widetilde{b}_{L}+\frac{m_{4}}{\sqrt{m_{4}^{2}+y_{L 4}^{2} f^{2}}} \widetilde{B}_{L},
\end{aligned}
$$

where we denoted by $\widetilde{B}$ the heavy mass eigenstate. The operators that induce a distortion of the $g_{b_{L}}$ coupling are trivially related to the ones that give the couplings of the $Z$ boson to the elementary $b_{L}$ and the composite $B_{L}$.

Notice that under the SM gauge group the $b_{L}$ and the $B_{L}$ fields have exactly the same charges as the physical $\widetilde{b}_{L}$, thus operators containing the covariant derivatives $D_{\mu} b_{L}$ and $D_{\mu} B_{L}$ do not give any distortion of the couplings. They only induce a rescaling of the canonical kinetic terms.

We start by analyzing the operators containing only $q_{L}$. As we said before, the elementary $q_{L}$ must necessarily be contracted with the spurion $\tilde{y}_{L}$, thus the relevant operators contain at least two spurion insertions. The $q_{L}$ field appears in the combination

$$
\left(U^{\dagger} \tilde{y}_{L} q_{L}\right)_{i, 5}
$$

\footnotetext{
${ }^{19}$ Multiple covariant derivatives can be also used (e.g. $\left.D_{\mu} D_{\nu} \psi\right)$ but they are not relevant for our analysis.
} 
where $i$ and 5 denote the uncontracted index of $U^{\dagger}$. The singlet component (index 5) does not contain the $b_{L}$ field, thus only the 4-plet part is relevant for our analysis. To get the $Z$ boson we must use the covariant derivative or the $d_{\mu}^{i}$ symbol. The index structure, however, does not allow us to construct an operator with $d_{\mu}^{i}$. The only possibility is

$$
i \bar{q}_{L} \tilde{y}_{L}^{\dagger} \gamma^{\mu} \tilde{y}_{L} D_{\mu} q_{L}
$$

which gives a renormalization of the usual $b_{L}$ kinetic term and does not induce a correction to the $g_{b_{L}}$ coupling. At order $y_{L}^{4}$ we get one operator that contributes to the distortion of the $Z \bar{b}_{L} b_{L}$ vertex:

$$
\mathcal{O}=i\left(\bar{q}_{L} y_{L}^{\dagger} \gamma^{\mu} y_{L} q_{L}\right)\left(U_{5 A}^{\dagger}\left(y_{L}\right)_{A}^{\alpha}\left(y_{L}^{\dagger}\right)_{B}^{\alpha} U_{B i} d_{\mu}^{i}\right)+\text { h.c. }
$$

In this case the 4 insertions of the $\tilde{y}_{L}$ spurion ensure that the corrections are finite at one loop.

We can now consider the operators containing only the composite 4-plet $\psi_{4}$. At least two spurion insertions are needed to generate an operator that breaks the $P_{L R}$ symmetry and corrects the $Z \bar{b}_{L} b_{L}$ vertex. Notice that if more than two spurions are present the operator corresponds to a finite one-loop contribution. If we want to classify possible divergent corrections, we can focus on the case with only two $\tilde{y}_{L}$ insertions.

From the previous discussion it follows that the only way to contract the $\tilde{y}_{L}$ spurions is

$$
U_{* A}^{\dagger}\left(y_{L}\right)_{A}^{\alpha}\left(y_{L}^{\dagger}\right)_{B}^{\alpha} U_{B *}
$$

where each $*$ denotes a free index which can correspond to a 4-plet or a singlet of $\mathrm{SO}(4)$. As we noticed before, operators containing $D_{\mu} \psi_{4}$ can only induce a rescaling of the canonical kinetic term for the $B$. Thus in order to obtain a distortion of the coupling with the $Z$ boson we need to include the $d_{\mu}^{i}$ symbol. It is easy to show that the expression $d_{\mu}^{i} \psi_{4}^{i}$ does not contain a term of the form $Z_{\mu} B$. This term can only be generated if the $d$-symbol index is contracted with the Goldstone matrix $U$. We are left with only one possibility:

$$
\mathcal{O}=i\left(\bar{\psi}_{4} \gamma^{\mu} \psi_{4}\right)\left(U_{5 A}^{\dagger}\left(y_{L}\right)_{A}^{\alpha}\left(y_{L}^{\dagger}\right)_{B}^{\alpha} U_{B i} d_{\mu}^{i}\right)+\text { h.c. . }
$$

With an explicit computation we find that this operator contains a coupling of the $B$ with the $Z$ boson:

$$
\mathcal{O} \supset\left(\sqrt{2} \sin ^{2}\left(\frac{\langle h\rangle}{f}\right)\right) \frac{g}{c_{w}} Z_{\mu} \bar{B} \gamma^{\mu} B .
$$

The operator in eq. (B.12) contains only two spurion insertions and corresponds to a logarithmically divergent contribution at one loop. After the rotation to the mass eigenstates a correction to the $Z \bar{b}_{L} b_{L}$ vertex is induced. Using eq. (B.7) we find that this correction arises at order $y_{L}^{4}$, as expected.

Finally we can consider the mixed operators containing one elementary and one composite field. The elementary $b_{L}$ must necessarily be contracted with a $\tilde{y}_{L}$ spurion. It is straightforward to show that at least two other spurion insertions are needed to construct an operator that can contribute to $\delta g_{b_{L}}$ and the associated one-loop corrections are finite. 
To conclude we summarize the results of this section. We found that the one-loop corrections to the $Z \bar{b}_{L} b_{L}$ can be logarithmically divergent. Moreover we showed that the divergence can only come from diagrams with two composite $B$ 's as external states. The contributions related to the elementary $b_{L}$ fields are instead always finite.

\section{Computation of the loop corrections to the $Z \bar{b}_{L} b_{L}$ vertex}

In this appendix we compute the one-loop corrections to the $Z \bar{b}_{L} b_{L}$ vertex. For simplicity we consider the limit in which the gauge couplings are sent to zero. This approximation is justified by the fact that, as in the SM, the most relevant contributions are related to the Yukawa interactions and not to the gauge couplings. ${ }^{20}$

The computation can be significantly simplified by using a consequence of the operator analysis presented in appendix B. We saw that, an operator can contribute to the distortion of the $Z \bar{b}_{L} b_{L}$ interaction only if it contains the CCWZ $d_{\mu}^{i}$ symbol. Moreover we found that the 4-plet index of $d_{\mu}$ must be necessarily contracted with the Goldstone matrix. By an explicit computation one easily finds that the combination $U_{A i} d_{\mu}^{i}$ contains the $Z$ boson always in association with the neutral Goldstone $\phi^{0}$ :

$$
U_{A i} d_{\mu}^{i} \supset-\frac{1}{\sqrt{2}}\left(\frac{g}{c_{w}} \sin \left(\frac{\langle h\rangle}{f}\right) Z_{\mu}+2 \partial_{\mu} \phi^{0}\right)
$$

where $\phi^{0}$ denotes the canonically normalized neutral Goldstone, $\phi^{0}=$ $-(f /\langle h\rangle) \sin (\langle h\rangle / f) \Pi_{3}$ (see appendix A). It is also straightforward to check that the covariant derivatives $D_{\mu} b_{L}$ and $D_{\mu} \psi_{4}$ do not contain any term of the form $\left(\partial_{\mu} \phi^{0}\right) b_{L}$. From these results it follows that we can extract the corrections to the $g_{b_{L}}$ coupling by computing the one loop contributions to the $\left(\partial_{\mu} \phi^{0}\right) \bar{b}_{L} \gamma^{\mu} b_{L}$ interaction. ${ }^{21}$

Notice that, thanks to the $P_{L R}$ symmetry under which $\phi^{0}$ is odd, the vertex $\left(\partial_{\mu} \phi^{0}\right) \bar{b}_{L} \gamma^{\mu} b_{L}$ is not present at tree level and this makes the computation of the $\left(\partial_{\mu} \phi^{0}\right) \bar{b}_{L} \gamma^{\mu} b_{L}$ one-loop corrections even simpler. Due to the presence of a tree-level $Z \bar{b}_{L} b_{L}$ vertex, the one loop renormalization of the $b_{L}$ must be taken into account to compute $\delta g_{b_{L}}$ in the standard way. In the case of the $\left(\partial_{\mu} \phi^{0}\right) \bar{b}_{L} \gamma^{\mu} b_{L}$ interaction, instead, the wave function renormalization does not induce a one-loop contribution, thus we only need to compute the vertex correction.

We parametrize the relevant Goldstone couplings in the following way:

$$
\begin{aligned}
\mathcal{L}= & \bar{T}_{i}\left(A_{i} \phi^{+}+i B_{i} \not \partial \phi^{+}\right) b_{L}+\text { h.c. } \\
& +\left(i C_{i j} \phi^{0} \bar{T}_{i} P_{L} T_{j}+\text { h.c. }\right)+\partial_{\mu} \phi^{0} \bar{T}_{i} \gamma^{\mu}\left(D_{i j}^{L} P_{L}+D_{i j}^{R} P_{R}\right) T_{j} \\
& +\bar{T}_{i}\left(i E_{i} \phi^{+} \phi^{0}+F_{i}^{+} \phi^{0} \not \partial \phi^{+}+F_{i}^{0} \phi^{+} \not \partial \phi^{0}\right) b_{L}+\text { h.c. },
\end{aligned}
$$

where we denoted by $T_{i}$ the charge $2 / 3$ states in the mass eigenbasis and $P_{L, R}$ are the left and right projectors. $\phi^{+}$and $\phi^{0}$ are the canonically normalized Goldstone fields, in

\footnotetext{
${ }^{20}$ We verified numerically in the model of ref. [27] that the corrections due to non-vanishing gauge couplings are small and can be safely neglected.

${ }^{21}$ Another proof of the correctness of this procedure was given in ref. [89], in which the two loop corrections to the $Z \bar{b}_{L} b_{L}$ vertex in the SM are computed.
} 

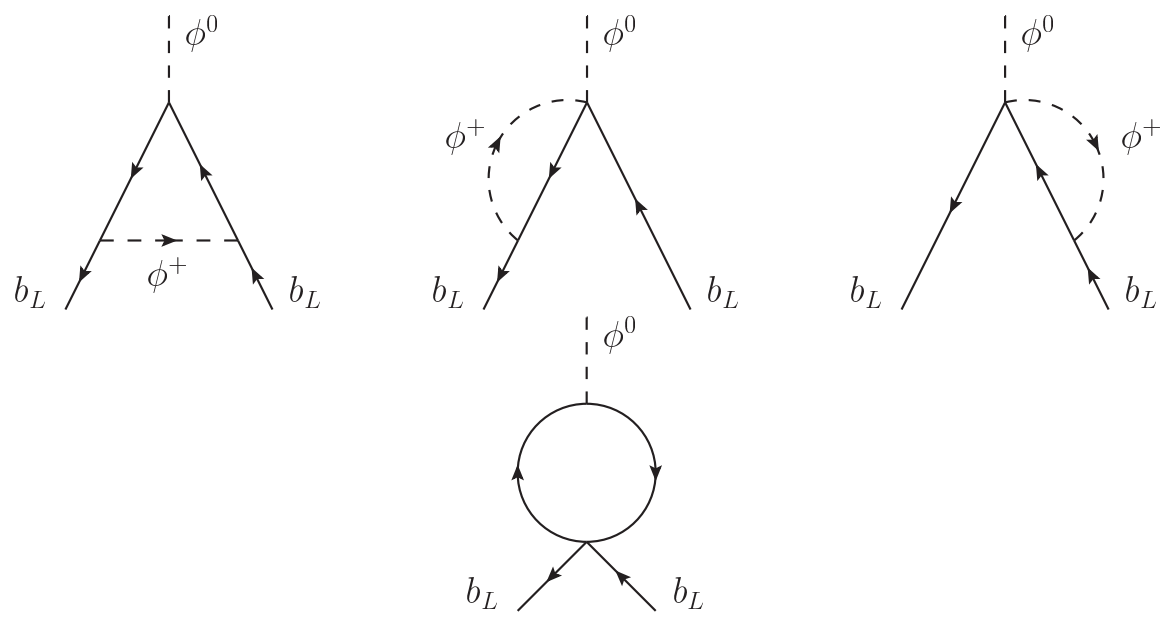

Figure 18. Topologies of the diagrams contributing to the $\left(\partial_{\mu} \phi^{0}\right) \bar{b}_{L} \gamma^{\mu} b_{L}$ interaction. The internal fermion lines are fields with electric charge $2 / 3$.

particular the charged Goldstone is given by $\phi^{+}=(f /\langle h\rangle) \sin (\langle h\rangle / f) h_{u}$ (see appendix A). Notice that, in the effective theory we considered in this paper, the $\phi^{0}$ Goldstone has no vertex that involves only charge $-1 / 3$ states. As a consequence the diagrams that give a correction to the $Z \bar{b}_{L} b_{L}$ vertex only contain charge $2 / 3$ fermions inside the loop.

As we discussed in the main text, corrections to the $g_{b_{L}}$ coupling can also be induced by 4 -fermion effective interactions. We parametrized them by the Lagrangian:

$$
\mathcal{L}^{4-\text { ferm. }}=G_{i j}^{L}\left[\bar{b}_{L}^{a} \gamma_{\mu} b_{L}^{a}\right]\left[\bar{T}_{i}^{b} \gamma^{\mu} P_{L} T_{j}^{b}\right]+G_{i j}^{R}\left[\bar{b}_{L}^{a} \gamma_{\mu} b_{L}^{a}\right]\left[\bar{T}_{i}^{b} \gamma^{\mu} P_{R} T_{j}^{b}\right]
$$

where $a$ and $b$ are color indices. For simplicity we consider only the color structure given in the previous formula. The results for different color structures only differ by an overall group theory factor.

The topologies of the diagrams that contribute to the $\left(\partial_{\mu} \phi^{0}\right) \bar{b}_{L} \gamma^{\mu} b_{L}$ interaction are shown in figure 18. The "triangle" topology and the diagrams with a loop on the external legs arise from the leading order terms in the composite Higgs effective Lagrangian. The 4 -fermion interactions, instead, generate the diagrams with a "bubble" topology. For our explicit computation we use dimensional regularization and we encode the divergent part in the parameter $\Delta \equiv 1 / \epsilon-\gamma+\log (4 \pi)$, where $\epsilon$ is defined by $d=4-2 \epsilon$. We denote the renormalization scale by $\mu$.

The correction to the $Z \bar{b}_{L} b_{L}$ vertex coming from the "triangle" diagrams is given by

$$
\begin{aligned}
& \delta g_{b_{L}}^{\text {triangle }}=\frac{f \sin (\langle h\rangle / f)}{64 \pi^{2}} \sum_{i, j}\left\{A_{j} A_{i}^{*}\left[D_{i j}^{R} I_{1}^{i j}+2 D_{i j}^{L} m_{i} m_{j} I_{2}^{i j}-C_{i j} m_{j}\left(I_{2}^{i j}-I_{4}^{i j}\right)-C_{i j}^{\dagger} m_{i}\left(I_{2}^{i j}+I_{4}^{i j}\right)\right]\right. \\
& +B_{j} B_{i}^{*}\left[D_{i j}^{R} m_{i} m_{j} I_{1}^{i j}-2 D_{i j}^{L} I_{3}^{i j}+\frac{1}{2} C_{i j} m_{i}\left(I_{1}^{i j}+I_{5}^{i j}\right)+\frac{1}{2} C_{i j}^{\dagger} m_{j}\left(I_{1}^{i j}-I_{5}^{i j}\right)\right] \\
& \left.+\operatorname{Re}\left[A_{j} B_{i}^{*}\left(C_{i j}^{\dagger}\left(3 I_{1}^{i j}-I_{5}^{i j}+1\right)+2 C_{i j} m_{i} m_{j} I_{4}^{i j}+2 D_{i j}^{R} m_{i} I_{1}^{i j}-2 D_{i j}^{L} m_{j}\left(2 I_{1}^{i j}+1\right)\right)\right]\right\},
\end{aligned}
$$


where we defined the $I_{1, \ldots, 5}$ functions as

$$
\begin{aligned}
I_{1}^{i j} & =\Delta+\frac{1}{2}-\frac{1}{m_{i}^{2}-m_{j}^{2}}\left[m_{i}^{2} \log \left(\frac{m_{i}^{2}}{\mu^{2}}\right)-m_{j}^{2} \log \left(\frac{m_{j}^{2}}{\mu^{2}}\right)\right], \\
I_{2}^{i j} & =\frac{1}{m_{i}^{2}-m_{j}^{2}} \log \left(\frac{m_{i}^{2}}{m_{j}^{2}}\right), \\
I_{3}^{i j} & =\left(m_{i}^{2}+m_{j}^{2}\right)(\Delta+1)-\frac{1}{m_{i}^{2}-m_{j}^{2}}\left[m_{i}^{4} \log \left(\frac{m_{i}^{2}}{\mu^{2}}\right)-m_{j}^{4} \log \left(\frac{m_{j}^{2}}{\mu^{2}}\right)\right], \\
I_{4}^{i j} & =\frac{1}{m_{i}^{2}-m_{j}^{2}}-\frac{m_{i}^{2}+m_{j}^{2}}{2\left(m_{i}^{2}-m_{j}^{2}\right)^{2}} \log \left(\frac{m_{i}^{2}}{m_{j}^{2}}\right), \\
I_{5}^{i j} & =\frac{m_{i}^{2}+m_{j}^{2}}{m_{i}^{2}-m_{j}^{2}}-\frac{2 m_{i}^{2} m_{j}^{2}}{\left(m_{i}^{2}-m_{j}^{2}\right)^{2}} \log \left(\frac{m_{i}^{2}}{m_{j}^{2}}\right) .
\end{aligned}
$$

The contribution from the diagrams with loops on the external legs is given by

$$
\begin{aligned}
\delta g_{b_{L}}^{\text {legs }}= & \frac{f \sin (\langle h\rangle / f)}{128 \pi^{2}} \sum_{i} \operatorname{Re}\left[4 F_{i}^{0} m_{i}\left(A_{i}^{*}+B_{i}^{*} m_{i}\right) I_{6}^{i}-E_{i}\left(A_{i}^{*}\left(I_{6}^{i}+1\right)-B_{i}^{*} m_{i}\left(I_{6}^{i}-1\right)\right)\right. \\
& \left.-F_{i}^{+} m_{i}\left(A_{i}^{*}\left(I_{6}^{i}-1\right)+B_{i}^{*} m_{i}\left(3 I_{6}^{i}-1\right)\right)\right],
\end{aligned}
$$

where $I_{6}$ is given by

$$
I_{6}^{i}=2 \Delta+2-2 \log \left(\frac{m_{i}^{2}}{\mu^{2}}\right) .
$$

Notice that in the effective theory we considered in this paper the two contributions $\delta g_{b_{L}}^{\text {triangle }}$ and $\delta b_{b_{L}}^{\text {legs }}$ are always finite.

Finally the contribution induced by the 4 -fermion interactions is given by

$$
\begin{aligned}
\delta g_{b_{L}}^{\text {bubble }}= & N_{c} \frac{f \sin (\langle h\rangle / f)}{32 \pi^{2}} \sum_{i, j}\left\{\left(D_{i j}^{L} G_{j i}^{L}+D_{i j}^{R} G_{j i}^{R}\right)\left(I_{3}^{i j}-\left(m_{i}^{2}+m_{j}^{2}\right) / 2\right)\right. \\
& \left.-\left(D_{i j}^{R} G_{j i}^{L}+D_{i j}^{L} G_{j i}^{R}\right) m_{i} m_{j}\left(2 I_{1}^{i j}+1\right)+\operatorname{Re}\left[C_{i j} G_{j i}^{L}-C_{i j}^{\dagger} G_{j i}^{R}\right] m_{i} I_{7}^{i j}\right\}(\mathrm{C} .8)
\end{aligned}
$$

where

$$
I_{7}^{i j}=2 \Delta+3-2 \frac{m_{i}^{2}}{m_{i}^{2}-m_{j}^{2}}-2 \frac{1}{\left(m_{i}^{2}-m_{j}^{2}\right)^{2}}\left[\left(m_{i}^{4}-2 m_{i}^{2} m_{j}^{2}\right) \log \left(\frac{m_{i}^{2}}{\mu^{2}}\right)+m_{j}^{4} \log \left(\frac{m_{j}^{2}}{\mu^{2}}\right)\right] .
$$

Differently from the first two classes of diagrams, in our effective theory the "bubble" diagrams can give a divergent contribution. This can happen if the $G_{i j}^{R}$ couplings are non-vanishing. The $G_{i j}^{L}$ couplings, instead, give rise only to finite corrections.

Open Access. This article is distributed under the terms of the Creative Commons Attribution License which permits any use, distribution and reproduction in any medium, provided the original author(s) and source are credited. 


\section{References}

[1] ATLAS collaboration, Observation of a new particle in the search for the Standard Model Higgs boson with the ATLAS detector at the LHC, Phys. Lett. B 716 (2012) 1 [arXiv:1207.7214] [INSPIRE].

[2] CMS collaboration, Observation of a new boson at a mass of $125 \mathrm{GeV}$ with the CMS experiment at the LHC, Phys. Lett. B 716 (2012) 30 [arXiv:1207.7235] [INSPIRE].

[3] L.J. Hall, D. Pinner and J.T. Ruderman, A natural SUSY Higgs near 126 GeV, JHEP 04 (2012) 131 [arXiv:1112.2703] [InSPIRE].

[4] D.B. Kaplan and H. Georgi, SU(2) × U(1) breaking by vacuum misalignment, Phys. Lett. B 136 (1984) 183 [INSPIRE].

[5] D.B. Kaplan, H. Georgi and S. Dimopoulos, Composite Higgs scalars, Phys. Lett. B 136 (1984) 187 [INSPIRE].

[6] H. Georgi, D.B. Kaplan and P. Galison, Calculation of the composite Higgs mass, Phys. Lett. B 143 (1984) 152 [inSPIRE].

[7] T. Banks, Constraints on $\mathrm{SU}(2) \times \mathrm{U}(1)$ breaking by vacuum misalignment, Nucl. Phys. B 243 (1984) 125 [INSPIRE].

[8] H. Georgi and D.B. Kaplan, Composite Higgs and custodial SU(2), Phys. Lett. B 145 (1984) 216 [INSPIRE].

[9] M.J. Dugan, H. Georgi and D.B. Kaplan, Anatomy of a composite Higgs model, Nucl. Phys. B 254 (1985) 299 [INSPIRE].

[10] R. Contino, Y. Nomura and A. Pomarol, Higgs as a holographic pseudo Goldstone boson, Nucl. Phys. B 671 (2003) 148 [hep-ph/0306259] [InSPIRE].

[11] K. Agashe, R. Contino and A. Pomarol, The minimal composite Higgs model, Nucl. Phys. B 719 (2005) 165 [hep-ph/0412089] [INSPIRE].

[12] S. Dimopoulos and J. Preskill, Massless composites with massive constituents, Nucl. Phys. B 199 (1982) 206 [INSPIRE].

[13] O. Matsedonskyi, G. Panico and A. Wulzer, Light top partners for a light composite Higgs, JHEP 01 (2013) 164 [arXiv:1204.6333] [INSPIRE].

[14] M. Redi and A. Tesi, Implications of a light Higgs in composite models, JHEP 10 (2012) 166 [arXiv:1205.0232] [INSPIRE].

[15] D. Marzocca, M. Serone and J. Shu, General composite Higgs models, JHEP 08 (2012) 013 [arXiv:1205.0770] [INSPIRE].

[16] A. Pomarol and F. Riva, The composite Higgs and light resonance connection, JHEP 08 (2012) 135 [arXiv:1205.6434] [INSPIRE].

[17] G. Panico, M. Redi, A. Tesi and A. Wulzer, On the tuning and the mass of the composite Higgs, JHEP 03 (2013) 051 [arXiv: 1210.7114] [INSPIRE].

[18] D. Pappadopulo, A. Thamm and R. Torre, A minimally tuned composite Higgs model from an extra dimension, JHEP 07 (2013) 058 [arXiv: 1303.3062] [INSPIRE].

[19] G. Giudice, C. Grojean, A. Pomarol and R. Rattazzi, The strongly-interacting light Higgs, JHEP 06 (2007) 045 [hep-ph/0703164] [INSPIRE]. 
[20] C. Dennis, M. Karagoz, G. Servant and J. Tseng, Multi-W events at LHC from a warped extra dimension with custodial symmetry, hep-ph/0701158 [INSPIRE].

[21] R. Contino and G. Servant, Discovering the top partners at the LHC using same-sign dilepton final states, JHEP 06 (2008) 026 [arXiv:0801.1679] [INSPIRE].

[22] J. Mrazek and A. Wulzer, A strong sector at the LHC: top partners in same-sign dileptons, Phys. Rev. D 81 (2010) 075006 [arXiv:0909.3977] [InSPIRE].

[23] A. Atre, M. Carena, T. Han and J. Santiago, Heavy quarks above the top at the Tevatron, Phys. Rev. D 79 (2009) 054018 [arXiv: 0806.3966] [InSPIRE].

[24] A. Atre et al., Model-independent searches for new quarks at the LHC, JHEP 08 (2011) 080 [arXiv: 1102.1987] [INSPIRE].

[25] R. Barcelo, A. Carmona, M. Chala, M. Masip and J. Santiago, Single vectorlike quark production at the LHC, Nucl. Phys. B 857 (2012) 172 [arXiv:1110.5914] [INSPIRE].

[26] A. Atre, M. Chala and J. Santiago, Searches for new vector like quarks: Higgs channels, JHEP 05 (2013) 099 [arXiv: 1302.0270] [INSPIRE].

[27] C. Anastasiou, E. Furlan and J. Santiago, Realistic composite Higgs models, Phys. Rev. D 79 (2009) 075003 [arXiv:0901.2117] [INSPIRE].

[28] J. Aguilar-Saavedra, Identifying top partners at LHC, JHEP 11 (2009) 030 [arXiv:0907.3155] [INSPIRE].

[29] New Physics Working Group collaboration, G. Brooijmans et al., New physics at the LHC. A Les Houches report: physics at TeV colliders 2009 - New Physics Working Group, arXiv: 1005.1229 [INSPIRE].

[30] G. Dissertori, E. Furlan, F. Moortgat and P. Nef, Discovery potential of top-partners in a realistic composite Higgs model with early LHC data, JHEP 09 (2010) 019 [arXiv: 1005.4414] [INSPIRE].

[31] T. Han, I. Lewis and Z. Liu, Colored resonant signals at the LHC: largest rate and simplest topology, JHEP 12 (2010) 085 [arXiv: 1010.4309] [INSPIRE].

[32] C. Bini, R. Contino and N. Vignaroli, Heavy-light decay topologies as a new strategy to discover a heavy gluon, JHEP 01 (2012) 157 [arXiv:1110.6058] [INSPIRE].

[33] N. Vignaroli, Discovering the composite Higgs through the decay of a heavy fermion, JHEP 07 (2012) 158 [arXiv: 1204.0468] [INSPIRE].

[34] K. Harigaya, S. Matsumoto, M.M. Nojiri and K. Tobioka, Search for the top partner at the LHC using multi-b-jet channels, Phys. Rev. D 86 (2012) 015005 [arXiv:1204.2317] [INSPIRE].

[35] J. Berger, J. Hubisz and M. Perelstein, A fermionic top partner: naturalness and the LHC, JHEP 07 (2012) 016 [arXiv: 1205.0013] [INSPIRE].

[36] S. Dawson and E. Furlan, A Higgs conundrum with vector fermions, Phys. Rev. D 86 (2012) 015021 [arXiv: 1205.4733] [INSPIRE].

[37] Y. Okada and L. Panizzi, LHC signatures of vector-like quarks, Adv. High Energy Phys. 2013 (2013) 364936 [arXiv:1207.5607] [INSPIRE].

[38] G. Cacciapaglia, A. Deandrea, L. Panizzi, S. Perries and V. Sordini, Heavy vector-like quark with charge 5/3 at the LHC, JHEP 03 (2013) 004 [arXiv:1211.4034] [INSPIRE]. 
[39] M. Buchkremer, G. Cacciapaglia, A. Deandrea and L. Panizzi, Model independent framework for searches of top partners, Nucl. Phys. B 876 (2013) 376 [arXiv:1305.4172] [INSPIRE].

[40] A. De Simone, O. Matsedonskyi, R. Rattazzi and A. Wulzer, A first top partner hunter's guide, JHEP 04 (2013) 004 [arXiv:1211.5663] [INSPIRE].

[41] J. Aguilar-Saavedra, R. Benbrik, S. Heinemeyer and M. Pérez-Victoria, A handbook of vector-like quarks: mixing and single production, arXiv:1306.0572 [INSPIRE].

[42] S. Gopalakrishna, T. Mandal, S. Mitra and G. Moreau, LHC signatures of warped-space vectorlike quarks, arXiv:1306.2656 [INSPIRE].

[43] CMS collaboration, Search for $T_{5 / 3}$ top partners in same-sign dilepton final state, CMS-PAS-B2G-12-012, CERN, Geneva Switzerland (2012).

[44] CMS collaboration, Inclusive search for a vector-like T quark by CMS, CMS-PAS-B2G-12-015, CERN, Geneva Switzerland (2012).

[45] ATLAS collaboration, Search for exotic same-sign dilepton signatures ( $b^{\prime}$ quark, $T_{5 / 3}$ and four top quarks production) in $4.7 \mathrm{fb}^{-1}$ of pp collisions at $\sqrt{s}=7 \mathrm{TeV}$ with the ATLAS detector, ATLAS-CONF-2012-130, CERN, Geneva Switzerland (2012).

[46] ATLAS collaboration, Search for anomalous production of events with same-sign dileptons and $b$ jets in $14.3 \mathrm{fb}^{-1}$ of pp collisions at $\sqrt{s}=8 \mathrm{TeV}$ with the ATLAS detector, ATLAS-CONF-2013-051, CERN, Geneva Switzerland (2013).

[47] R. Barbieri, B. Bellazzini, V.S. Rychkov and A. Varagnolo, The Higgs boson from an extended symmetry, Phys. Rev. D 76 (2007) 115008 [arXiv:0706.0432] [InSPIRE].

[48] R. Contino, The Higgs as a composite Nambu-Goldstone boson, arXiv:1005.4269 [INSPIRE].

[49] J. Espinosa, C. Grojean, M. Muhlleitner and M. Trott, First glimpses at Higgs' face, JHEP 12 (2012) 045 [arXiv: 1207.1717] [INSPIRE].

[50] A. Falkowski, F. Riva and A. Urbano, Higgs at last, arXiv:1303.1812 [INSPIRE].

[51] M. Ciuchini, E. Franco, S. Mishima and L. Silvestrini, Electroweak precision observables, new physics and the nature of a $126 \mathrm{GeV}$ Higgs boson, JHEP 08 (2013) 106 [arXiv:1306. 4644] [INSPIRE].

[52] K. Agashe, A. Delgado, M.J. May and R. Sundrum, RS1, custodial isospin and precision tests, JHEP 08 (2003) 050 [hep-ph/0308036] [INSPIRE].

[53] K. Agashe and R. Contino, The minimal composite Higgs model and electroweak precision tests, Nucl. Phys. B 742 (2006) 59 [hep-ph/0510164] [INSPIRE].

[54] M. Gillioz, A light composite Higgs boson facing electroweak precision tests, Phys. Rev. D 80 (2009) 055003 [arXiv:0806.3450] [INSPIRE].

[55] P. Lodone, Vector-like quarks in a 'composite' Higgs model, JHEP 12 (2008) 029 [arXiv:0806.1472] [INSPIRE].

[56] A. Pomarol and J. Serra, Top quark compositeness: feasibility and implications, Phys. Rev. D 78 (2008) 074026 [arXiv:0806.3247] [INSPIRE].

[57] M. Gillioz, R. Grober, C. Grojean, M. Muhlleitner and E. Salvioni, Higgs low-energy theorem (and its corrections) in composite models, JHEP 10 (2012) 004 [arXiv:1206.7120] [INSPIRE]. 
[58] R. Barbieri, D. Buttazzo, F. Sala, D.M. Straub and A. Tesi, A 125 GeV composite Higgs boson versus flavour and electroweak precision tests, JHEP 05 (2013) 069 [arXiv:1211.5085] [INSPIRE].

[59] D.B. Kaplan, Flavor at SSC energies: a new mechanism for dynamically generated fermion masses, Nucl. Phys. B 365 (1991) 259 [inSPIRE].

[60] R. Contino, T. Kramer, M. Son and R. Sundrum, Warped/composite phenomenology simplified, JHEP 05 (2007) 074 [hep-ph/0612180] [INSPIRE].

[61] S.R. Coleman, J. Wess and B. Zumino, Structure of phenomenological Lagrangians. 1, Phys. Rev. 177 (1969) 2239 [INSPIRE].

[62] C.G. Callan Jr., S.R. Coleman, J. Wess and B. Zumino, Structure of phenomenological Lagrangians. 2, Phys. Rev. 177 (1969) 2247 [InSPIRE].

[63] R. Contino, L. Da Rold and A. Pomarol, Light custodians in natural composite Higgs models, Phys. Rev. D 75 (2007) 055014 [hep-ph/0612048] [INSPIRE].

[64] G. Panico, E. Ponton, J. Santiago and M. Serone, Dark matter and electroweak symmetry breaking in models with warped extra dimensions, Phys. Rev. D 77 (2008) 115012 [arXiv:0801.1645] [INSPIRE].

[65] G. Panico, M. Safari and M. Serone, Simple and realistic composite Higgs models in flat extra dimensions, JHEP 02 (2011) 103 [arXiv: 1012.2875] [INSPIRE].

[66] G. Panico and A. Wulzer, The discrete composite Higgs model, JHEP 09 (2011) 135 [arXiv:1106.2719] [INSPIRE].

[67] S. De Curtis, M. Redi and A. Tesi, The 4D composite Higgs, JHEP 04 (2012) 042 [arXiv:1110.1613] [INSPIRE].

[68] M.E. Peskin and T. Takeuchi, Estimation of oblique electroweak corrections, Phys. Rev. D 46 (1992) 381 [INSPIRE].

[69] R. Barbieri, A. Pomarol, R. Rattazzi and A. Strumia, Electroweak symmetry breaking after LEP-1 and LEP-2, Nucl. Phys. B 703 (2004) 127 [hep-ph/0405040] [INSPIRE].

[70] M. Baak et al., The electroweak fit of the Standard Model after the discovery of a new boson at the LHC, Eur. Phys. J. C 72 (2012) 2205 [arXiv:1209.2716] [InSPIRE].

[71] A. Orgogozo and S. Rychkov, The S parameter for a light composite Higgs: a dispersion relation approach, JHEP 06 (2013) 014 [arXiv: 1211.5543] [INSPIRE].

[72] L. Lavoura and J.P. Silva, The oblique corrections from vector-like singlet and doublet quarks, Phys. Rev. D 47 (1993) 2046 [inSPIRE].

[73] M.S. Carena, E. Ponton, J. Santiago and C.E. Wagner, Light Kaluza Klein states in Randall-Sundrum models with custodial SU(2), Nucl. Phys. B 759 (2006) 202 [hep-ph/0607106] [INSPIRE].

[74] M.S. Carena, E. Ponton, J. Santiago and C. Wagner, Electroweak constraints on warped models with custodial symmetry, Phys. Rev. D 76 (2007) 035006 [hep-ph/0701055] [INSPIRE].

[75] M. Golden and L. Randall, Radiative corrections to electroweak parameters in technicolor theories, Nucl. Phys. B 361 (1991) 3 [INSPIRE].

[76] R. Barbieri, G. Isidori and D. Pappadopulo, Composite fermions in electroweak symmetry breaking, JHEP 02 (2009) 029 [arXiv:0811.2888] [INSPIRE]. 
[77] J. Elias-Miró, J. Espinosa, E. Masso and A. Pomarol, Renormalization of dimension-six operators relevant for the Higgs decays $h \rightarrow \gamma \gamma, \gamma Z$, JHEP 08 (2013) 033 [arXiv:1302.5661] [INSPIRE].

[78] R. Contino, M. Ghezzi, C. Grojean, M. Muhlleitner and M. Spira, Effective Lagrangian for a light Higgs-like scalar, JHEP 07 (2013) 035 [arXiv: 1303.3876] [INSPIRE].

[79] B. Batell, S. Gori and L.-T. Wang, Higgs couplings and precision electroweak data, JHEP 01 (2013) 139 [arXiv:1209.6382] [INSPIRE].

[80] D. Guadagnoli and G. Isidori, $B R\left(B_{s} \rightarrow \mu^{+} \mu^{-}\right)$as an electroweak precision test, arXiv:1302. 3909 [INSPIRE].

[81] K. Agashe, R. Contino, L. Da Rold and A. Pomarol, A custodial symmetry for Z $b \bar{b}$, Phys. Lett. B 641 (2006) 62 [hep-ph/0605341] [INSPIRE].

[82] J. Mrazek et al., The other natural two Higgs doublet model, Nucl. Phys. B 853 (2011) 1 [arXiv: 1105.5403] [INSPIRE].

[83] CMS collaboration, Measurement of the single-top-quark t-channel cross section in pp collisions at $\sqrt{s}=7 \mathrm{TeV}$, JHEP 12 (2012) 035 [arXiv: 1209.4533] [INSPIRE].

[84] F. del Aguila, M. Pérez-Victoria and J. Santiago, Effective description of quark mixing, Phys. Lett. B 492 (2000) 98 [hep-ph/0007160] [INSPIRE].

[85] F. del Aguila, M. Pérez-Victoria and J. Santiago, Observable contributions of new exotic quarks to quark mixing, JHEP 09 (2000) 011 [hep-ph/0007316] [INSPIRE].

[86] J. Aguilar Saavedra and M. Pérez-Victoria, Top couplings and top partners, J. Phys. Conf. Ser. 452 (2013) 012037 [arXiv: 1302.5634] [INSPIRE].

[87] B. Grzadkowski, M. Iskrzynski, M. Misiak and J. Rosiek, Dimension-six terms in the Standard Model Lagrangian, JHEP 10 (2010) 085 [arXiv:1008.4884] [INSPIRE].

[88] J. Aguilar-Saavedra, M. Fiolhais and A. Onofre, Top effective operators at the ILC, JHEP 07 (2012) 180 [arXiv: 1206.1033] [INSPIRE].

[89] R. Barbieri, M. Beccaria, P. Ciafaloni, G. Curci and A. Vicere, Two loop heavy top effects in the Standard Model, Nucl. Phys. B 409 (1993) 105 [inSPIRE]. 